\title{
LASER SPECTROSCOPY
}

Assessment of Research Needs for

Laser Technologies Applied to

Advanced Spectroscopic Methods

May 1990

Prepared for:

U.S. Department of Energy

Office of Energy Research

Office of Program Analysis

Washington, DC 20545

Under Contract No. DE-AC01-88ER30131 


\section{DISCLAIMER}

This report was prepared as an account of work sponsored by an agency of the United States Government. Neither the United States Government nor any agency thereof, nor any of their employees, make any warranty, express or implied, or assumes any legal liability or responsibility for the accuracy, completeness, or usefulness of any information, apparatus, product, or process disclosed, or represents that its use would not infringe privately owned rights. Reference herein to any specific commercial product, process, or service by trade name, trademark, manufacturer, or otherwise does not necessarily constitute or imply its endorsement, recommendation, or favoring by the United States Government or any agency thereof. The views and opinions of authors expressed herein do not necessarily state or reflect those of the United States Government or any agency thereof. 


\section{DISCLAIMER}

Portions of this document may be illegible in electronic image products. Images are produced from the best available original document. 


\section{EXPERT TECHNICAL PANEL}

The following panel of scientists evaluated the research needs for laser technologies applicable to programs of the Office of Health and Environmental Research of the Office of Energy Research, Department of Energy. This project was conducted under contract with the Office of Program Analysis, Department of Energy, and was carried out under the leadership of G. Samuel Hurst (Principal Investigator) of Consultec Scientific, Inc.

\section{Main Panel}

Prof. Keith Boyer

Department of Physics

University of I11inois

Chicago, IL 60680

Dr. Robert L. Byer

Ginzton Laboratory of Physics

Stanford University

Stanford, CA 94305

Dr. Ira W. Levin

Bldg. 2, Room Bl-27

National Institutes of Health

Bethesda, MD 20892

Dr. David M. Lubman

Chemistry Department

University of Michigan

930 North University

Ann Arbor, MI 48109

Dr. Stephen R. Lundeen

Department of Physics

Univ. of Notre Dame

Notre Dame, IN 46556
Dr. N. Omenetto

Commission EC/JRC

Environment Institute

Chemistry Division

I-21020 Ispra, ITALY

Dr. Gary Salzman

MS M880

Los Alamos National Lab.

P. 0. Box 1663

Los Alamos, NM 87545

Dr.John Travis

B1dg. 222, Room A223

National Institute of

Standards \& Technology

Gaithersburg, MD 20899

Dr. Nicholas Winograd

Department of Chemistry

Pennsylvania State Univ.

University Park, PA 16802

Special Consultants

Prof. N. Bloembergen

231 Pierce Hall DAS

Harvard University

29 0xford Street

Cambridge, MA 02138

Prof. Yuan T. Lee

Chemistry Department

University of California

Berkeley, CA 94720
Prof. A. L. Schawlow

Department of Physics

Stanford University

Stanford, CA 94305 


\section{ACKNOWLEDGEMENTS}

In carrying out the work described in this report, the Consultec Scientific, Inc. staff and its expert panel interacted with a large number of researchers and research managers throughout the government, industry, and private sectors of the laser community. The assistance of these individuals in providing us with information and in cooperating with us in other ways is gratefuliy acknowledged.

Consultec Scientific, Inc. is pleased to acknowledge the expert panel and to thank those outstanding scientists for the considerable time which had to be taken from their busy schedules. The conclusions and overall research priorities described in the executive summary reflect the consensus reached at the final meeting of the Expert Scientific Pane1.

Special thanks are also due to Profs. Nicolaas Bloembergen,

Yuan T. Lee, and Arthur L. Schawlow who served as special consultants to this project. Also, the many valuable comments and suggestions from a group of peers (see Appendix III) are gratefully acknowledged.

Finally, we thank Dr. Gerald Goldstein for briefing the panel on the OHER programs and Dr. Robert Rosentha7, DOE Project Manager, for his insightful coordination of the work. 
Page No.

EXECUTIVE SUMMARY

viii

RECOMMENDATIONS

ix

1. INTRODUCTION

2. SUMMARY OF THE OFFICE OF HEALTH AND ENVIRONMENTAL

RESEARCH (OHER) PROGRAMS: A PERSPECTIVE ON THE

USE OF LASER SPECTROSCOPY

2.1. Introduction .............. 3

2.2. Source and Dose Determination ......... 3

2.3. Environmental Processes and Effects ....... 3

2.4. Health Effects ............... 6

2.5. Nuclear Medicine Applications .......... 7

3. STATUS OF RELEVANT LASER TECHNOLOGIES . . . . . . . 8

3.1. Introduction ............... 8

3.2. Sources ................. 8

3.2.1. Gas and Liquid Lasers ............ 8

3.2.2. Solid-State Lasers ......... 11

3.2.3. Free-Electron Lasers ........ 13

3.2.4. X-Ray Lasers ........... 13

3.2.5. Non 7 inear Frequency Extension ..... 14

3.3. Detectors for Laser Spectroscopy . . . . . . 16

3.3.1. Photon Detectors .......... 16

3.3.2. Ionization Detectors ........ 17

3.4. Imaging ..................... 18

3.5. Reservoirs ........................ 18

3.5.1. Introduction .......... 18 
3.5.2. Classical Atomic Spectroscopic Methods

3.5.3. Glow Discharges ........... 19

3.5.4. Laser-Induced Evaporation ...... 20

3.5.5. Particle-Induced Vaporization ..... 20

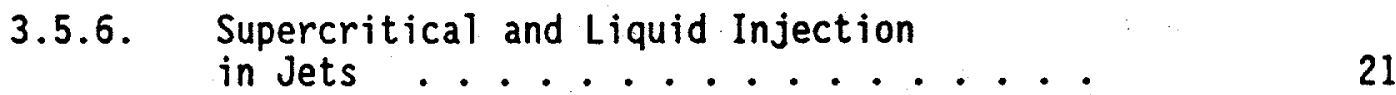

3.5.7. Other Methods ........... 21

3.6. Systems ........................... 21

3.6.1. Laser Mass Spectroscopy Systems . . . . 21

3.6.2. Laser Chromatographic Systems . . . . 23

4. STATUS OF LASER SPECTROSCOPY ................ 27

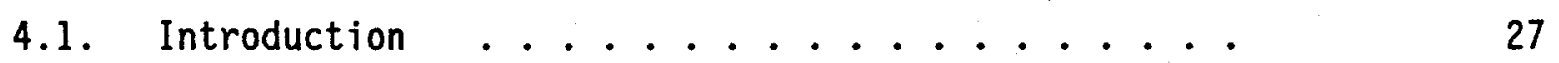

4.2. Fluorescence ...................... 28

4.2.1. Introduction ........... 28

4.2.2. Atomic Fluorescence ......... 28

4.2.3. Gas Phase Molecular Fluorescence . . . 29

4.2.4. Condensed Phase Fluorescence ...... 30

4.3. Absorption Spectroscopy ............. 31

4.4. Raman Spectroscopy .............. 31

4.5. Resonant and Multiphoton Ionization ..................
of Atoms

4.6. Multiphoton Ionization of Molecules ....... 37

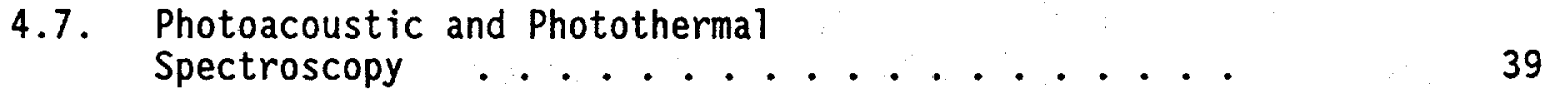

4.7.1. Introduction .......... 39

4.7.2. Photoacoustic Spectroscopy ...... 39

4.7.3. Photothermal Spectroscopy ........ 40 
5. POTENTIAL IMPACTS ON THE OHER PROGRAMS ......... 43

5.1. Introduction ................ 43

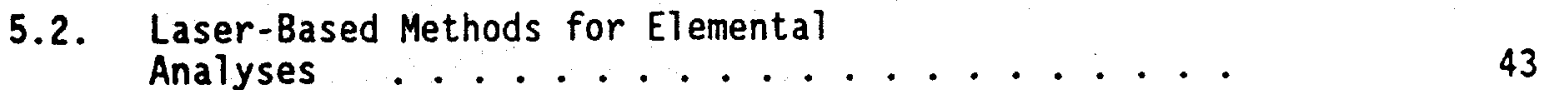

5.3. Environmental Research ............ 45

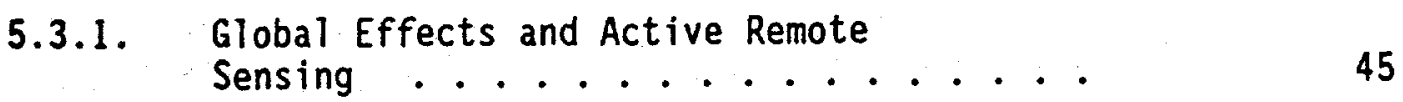

5.3.2. Transport Studies Involving
Actinides and Heavy Elements ....... 48

5.3.3. Fossil Fuel Pollutants ........ 49

5.3.4. Chemical Sensors (Optrodes) ....... 50

5.4. Biological Research ............. 51

5.4.1. Imaging ............ 51

5.4.2. Fast Chemical Kinetics ........ 52

5.4.3. Flow Cytometry ........... 54

5.4.4. Biosensors ........... 56

5.4.5. Ultrasensitive Detection and
Analysis for Biological Research ....

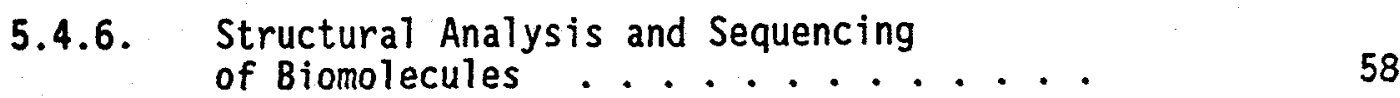

5.4.7. Genome Sequencing .......... 59

5.5. Nuclear Medicine Using Stable Isotopes ...... 60

5.6. Radiation Dosimetry ............. 61

APPENDIX I LASER ASSESSMENT STUDY ACRONYMS . . . 66

APPENDIX II INTRODUCTION TO TERMINOLOGY ...... 68

APPENDIX III $\quad$ PEER REVIEW GROUP ............ 73 


\section{EXECUTIVE SUMMARY}

The Department of Energy (DOE)* recognizes that new developments in laser technology and laser spectroscopy can substantially improve the ability to carry out the mission of its Office of Health and Environmental Research (OHER). In brief, the mission of OHER is to support programs of research which allow DOE to understand and anticipate long term effects upon human health and the environment from the production and utilization of alternate forms of energy, and to apply the department's unique capabilities to solve numerous problems in biology and medicine.

For some years, the OHER program has included some support for the development of laser technology, and especially laser spectroscopy. This support has provided the basis for the demonstration of laser systems of remarkable sensitivity for the detection of chemical substances at the atomic and the molecular levels. At the present time, both laser technology in general and laser spectroscopy in particular are surging ahead at an unprecedented rate. Thus, DOE requested that its Office of Program Analys is study the opportunities in this laser revolution that would further enhance the role of laser spectroscopy in the OHER mission. The purpose for this study is to provide an independent assessment of the long-term research needs for laser technologies applied to advanced spectroscopic methods and to address the content and priority of research needs for the next 5 to 10 years.

This DOE study was managed by Consultec Scientific, Inc. who furnished from its staff the Principal Investigator who, in turn, coordinated the enthusiastic efforts of a group of consultants consisting of some of the world's best scientists in the development and use of laser technology for spectroscopic purposes. During the course of its study, this expert panel, took a global view of laser science to ident ify recent developments which could substantially improve the ability of OHER to perform its increasingly important mission.

The panel made six specific recommendations which dealt with three important areas. First the panel recommends that OHER closely monitor and be prepared to use the advances now being made in solid-state laser technology. These advances, comparable in nature to the revolution which began during the 1950's in solid-state electronics, will radically improve present-day laser technology. Secondly, the panel addressed the use of this advanced technology to maintain the preeminent position which OHER has already created for itself in the development of selective and sensitive instruments for the analysis of atomic and molecular substances and to extend the use of these to measure chemical pollutants in air, soil, and water. Finally, another area of the recommendations dealt with the use of lasers to determine structural and dynamical features of macromolecules and especially to develop $X$-ray lasers and other imaging techniques, including holographic ones, for sequencing DNA and the human genome.

Improved analytical techniques based on laser spectroscopy can help DOE to understand and anticipate the effects upon human health and the environment from by-products of the industrial society and can produce numerous other benefits, such as a more complete understanding of biological systems for diagnosis and cure of diseases and for the biotechnology industry. 
The recommendations of the panel are presented in a prioritized list which we believe will produce the maximum beneficial impact of new developments in lasers upon the research programs of OHER.

The rationale behind each recommendation is based on two factors. First, each recommendation is considered to be a timely opportunity to take advantage of the recent surge of knowledge in laser science and technology to further the mission of OHER. The second factor is based on feasibility; we believe that each recommendation is scientifically sound and technically feasible. This list of recommendations is the result of considerable study and reflection by the expert panel in which there was near unanimity of opinion on the ranking of the recommendations. These recommendations are based on the in-depth discussions of Sections 3, 4, and 5. Section 5, in particular, contains a number of suggestions which may be very useful to those doing research for application of lasers to programs within the OHER mission. These "embedded suggestions". represent a broader base from which the prioritized recommendations were drawn. The establishment of a scale for the prioritization is difficult. For instance, the first recommendation is dominant to the extent that it would, in fact, enhance each of the other recommendations.

The last part of this section provides fundamental research recommendations dealing with more basic research that supports the recommended technologies. These fundamental research recommendations are not prioritized.

1. Accommodate the rapid developments now occurring in diode and solid-state laser technology to fit the needs of laser spectroscopy.

Revolutionary developments are occurring in diode and solid-state laser technology (see Sections 3.1 and 3.2).
We recommend that OHER support the application of solid-state laser technology to its programs and carefully monitor the development of these new lasers. Based upon developments by others, it may be cost effective by 1992 to generate visible radiation for high definition television projection, using solid-state lasers. By 1995 it should be less expensive to generate ultraviolet radiation using (instead of excimer lasers) diode-pumped, solid-state lasers followed by nonlinear frequency conversion. By that year, diode-pumped, solid-state laser sources up to the kilowatt average-power level will be available for application to chemical processing, materials processing, cutting, welding, and spectroscopic applications. We can expect dramatic reductions in cost and volume and, at the same time, improvements in reliability and convenience of use.

Clear cut needs for OHER's various missions include tunable lasers in the infrared (IR), visible, and ultraviolet (UV) regions as diagnostic tools used in monitoring the environment for contaminants associated with energy production. Methods which would benefit from the timely application of solidstate lasers include resonance ionization spectroscopy, other methods of sensitive detection, and techniques such as $X$-ray holography. See Section 5 for more technical details.

\section{Develop improved methods of molecular structural analyses and sequencing, based on capabilities in laser technology and spectroscopy.}

We recommend that OHER further develop improved laser methodologies for molecular structural analysis. Development of these methodologies would have at least two important impacts: (a) sequencing information for DNA and proteins and (b) examination of macromolecular structures in general.

There are several important research priorities for sequencing. Laser-based 
mass spectrometric techniques could play an increasingly important role in sequencing because they provide exact mass analysis of the sequence components. Rapid sequencing techniques using laser mass spectrometry will be important for minimizing errors inherent in other methods. The use of flow cytometry and other rapid flow methods in combination with selective and sensitive detection using lasers will enhance the speed of sequencing for such formidable tasks as DNA structural analysis. Direct imaging techniques such as X-ray microholography for rapid identification and resolution of the base units are expected to play an important role in genome sequencing.

Understanding the arrangement of large assemblies such as membranes, viruses, and intact DNA is an exciting area in the realm of macromolecular structures. The relationship between structure and activity of complex biological molecules such as DNA could be explored using laser techniques. These techniques include light scattering and $X$-ray holography (see Section 5.4.1).

3. Improve the capability for atomic and molecular detection, including isotope selectivity through laser methods.

The applications stemming from the detection of atoms and molecules ionized by lasers are growing rapidly (see Sections 4 and 5), and we recommend that this successful on-going OHER effort be continued with a high priority. With the new breed of continuous wave solid-state lasers, for example, it should be possible to selectively ionize stable nuclear isotopes for detection in medical and environmental tracing. New, highly reliable, pulsed vacuum-ultraviolet (VuV) lasers could be used for single-photon (nonselective) ionization of molecules and for a resonance excitation step in the selective ionization of inert gases. Isotopes of the inert gases are key to understanding basic environmental questions such as the transport of groundwater.

Novel methods for introducing samples into the gas phase continue to be discovered, and further research initiatives in this area should be pursued. Indications that it is possible to volatilize solid-state atoms or molecules directly from their environmental matrix by laser- or ionbeam-induced desorption are encouraging.

The pieces for making major advances in the selective detection of a wide variety of molecules are in place. By controlling the wavelength and the number of absorbed photons, it is possible to optimize sensitivity, selectivity, and structural information for rather complex molecules. The development of new methods of analyses of environmentally important mixtures, the selective detection of DNA-bound nucleic acids, and the detection of laser-ionized molecules at atmospheric pressure are three diverse examples of possible payoffs.

4. Develop coherent detection methods, imaging techniques, and holographic methods for biological and environmental applications.

We recommend supporting development of coherent detection techniques because of their applications to widely divergent fields such as remote sensing and new forms of microscopy. The following topics are examples of important applications of coherent detection techniques.

High intensity laser sources (Section 3.2), including pulsed lasers, have been developed with almost arbitrarily narrow 1 inewidths in the IR, visible, and UV spectral regions. This makes possible high precision Doppler measurements of wind and cloud velocities over long distances. It also permits remote sensing of contamination in the atmosphere. Since these techniques are a) so of direct importance to the military, the National Aeronautics and Space Administration (NASA), and other government agencies, it is recommended that OHER monitor these developments and fund particular measurements needed which are not supported by other agencies.

New developments in these coherent sources and detection methods (Sections 3.2.4 and 5.4.1) offer great potential 
for revealing information on biological systems of direct interest to OHER. In particular, this capability should reveal the exact way nuclear radiation affects different tissue and will thus improve the understanding of the effects of lowlevel and high-level exposures. An atomic tagging technique based on $X$-ray holography may make it possible to read rapidly the sequence of base pairs in DNA and to study its higher organizational structure. The $X$-ray holography programs are so new that they are not heavily funded at this time, and it appears that OHER is the appropriate agency to capitalize on this technology and encourage its more rapid development.

There is currently an important window of opportunity to develop new approaches to imaging biological samples with increased spatial resolution. Coherent confocal microscopy has recently demonstrated edge resolutions that are a factor-of-two improvement over incoherent microscopy. Similarly, sub-wavelength microscopy methods are being proposed that depend upon near-field imaging. With the appearance of X-ray 1asers, the development of an imaging $X$-ray microscope will soon be possible. Finally, the gradient imaging approach making use of gradient fields such as the successfully demonstrated magnetic resonance imaging (MRI) could be extended into the optical frequency region. Support by the Department of Energy provides an opportunity for the application of these new microscopic tools for biological research.

5. Improve laser methods for detection of chemical pollutants in air. soil, and water.

We recommend emphasizing the improvement of methods for global measurements of atmospheric parameters since they allow the study of the impact of energy consumption on the global ecology. Laser radiation in the UV, visible, and IR spectral regions allows selective excitation and detection of molecular, atomic, and ionic species. During the past 20 years, advantage has been taken of improved laser sources to discover and implement new methods for the detection of chemicals in the environment, (Sections 4 and 5.3). Examples include absorption spectroscopy, fluorescence spectroscopy, resonance ionization spectroscopy, ionization mass spectroscopy, and Raman spectroscopy. Further, with the advances in tunable laser sources, coherent forms of these spectroscopies have been developed that allow long range or remote sensing of chemical species. As noted in the Brinkman report on Physics in the 1990's, in the past decade new forms of spectroscopy have been discovered at such an astonishing rate that the terms describing the spectroscopies were not even known one decade earlier. Continued support of research by the Department of Energy provides an opportunity to discover and develop new forms of laser spectroscopy that are applicable to the detection of chemical species in the air, soil, and water. The continued development of the laser sources in wavelength, power, coherence, and efficiency makes this area of research very promising.

\section{Improve detectors and digital} data processing as applied to laser technology and to imaging techniques such as tomography.

We recommend investing resources in improving optical detectors, particularly small solid-state devices for wavelengths ranging from the UV to the IR. Techniques such as capillary zone electrophores is (CZE) make it possible to resolve attomole quantities of proteins. Flow cytometers can be used to examine picoliter quantities of material. Better detectors (Section 3.3 and 5.4) are needed to support these state-of-the-art techniques.

There are opportunities for the coupling of advances in computational techniques, such as parallel processing, to enhance the capability of optical detectors. In particular, the extensive development of signal and image processing for the field of high-energy physics and in the space program may be very useful for OHER's various missions. 
Fundamental Research Recommendations.

In addition to the above suggestions that deal with specific instrumentation, the panel has several general recommendations for programs that are more fundamental in nature and tend to support the technologies recommended above. These general recommendations are given below.

Laser science is still young; current understanding is by no means complete. Under these circumstances, the committee strongly endorses OHER's policy of supporting basic research in selected areas of potentially great impact on its mission. The continuation, or expansion, of this policy is seen as quite likely to pay substantial dividends.

The rapid growth and wide use of laser technology over the past 25 years has led to a great deal of new fundamental knowledge about light and matter and their interactions. The characteristics of laser light are so dramatically different from conventional light sources that scientists have regularly succeeded in accomplishments which were inconceivable before lasers. In retrospect, many of these accomplishments required innovative changes in our thinking about the basic physical processes involved.

Laser technology continues to advance rapidly, making accessible new phenomena which could never before be studied in the laboratory. This explosion of new knowledge is very likely to continue or accelerate in the future. Since many of the processes which are being seen in this "new light" have very strong potential for applications which are relevant to the OHER mission, OHER has recognized this aspect of laser science by supporting a selected number of research programs which study problems related to important applications at a very fundamental level. The small number of projects supported on this bas is have produced scientific results of extremely high quality, and contributed in a very noticeable way to the generation of this new knowledge. There is, however, still a great deal to be learned. There are many questions of potentially high impact on OHER interests where our understanding of the fundamental processes is incomplete or even totally absent. Some of these which the panel identified are given below:

\section{Photo-fragmentation of molecules.}

When high intensity lasers interact with a gaseous sample of molecules, many of the molecules may break apart into smaller fragments, either neutral or charged. Unlike conventional light sources, the exciting laser can cause sufficient population of excited levels of the molecule so that the fragmentation process may proceed in new and unexpected ways. If this process could be understood in detail so that it could be controlled and utilized, it could contribute in many ways to questions of interest to OHER.

\section{Laser ablation of solids.}

A laser beam incident on a solid sample can readily vaporize a portion of the sample. This process has the potential to be very useful, for instance, in analyzing the composition of the sample. Currently, however, the physical processes involved in such laser ablation are not sufficiently understood to make this possible. In what form are the fragments emitted? How is the answer affected by the laser characteristics?

\section{U1trahigh intensity laser fields.}

The achievable intensity from laser sources (power per unit area) has grown steadily since the laser's invention, and is likely to continue to do so. Current intensity levels $\left(-10^{15} \mathrm{~W} / \mathrm{cm}^{2}\right)$ are high enough that the optical electric fields in the laser beam are already comparable to the ordinary electric fields which hold atoms and molecules together. It is already clear that completely new physical ideas and models will be required to understand the interactions of atoms and molecules with lasers of this intensity, and that much effort will 
be required to develop and test these ideas. The potential for important applications is so high that these questions should be answered.

Fundamental research for laser source development.

Development of new lasers often requires answering basic scientific questions in diverse scientific disciplines, such as atomic and molecular physics, solid-state physics, non-linear optics, etc. Support of basic research related to laser source development, therefore, advances basic knowledge in all these areas and, in addition, leadsto improved lasers which are themselves valuable research tools.
Laser cooling, trapping, and manipulation of particles.

Within the last few years, it has been demonstrated experimentally that laser light can be used to suspend atoms in vacuum and cool them to very low temperatures $\left(10^{-3} \mathrm{~K}\right)$. Many other forms of mechanical manipulation of individual atoms with lasers also appear to be feasible. The basic physical principles involved in these new observations are not yet fully understood, al though they have potential for application in areas of sensitive detection which are of interest to OHER. Laser tweezers are already being used to manipulate small objects such as cells or bacteria. 


\section{INTRODUCTION}

The Atomic Energy Commission charter [The Atomic Energy Act of 1946 (PL 79585)] included a comprehensive program of applied and basic biological research relating to the utilization of fissionable and radioactive materials for medical, biological, and health purposes. As the DOE was created through various legislative acts to broaden the charter to other forms of energy production, the responsibilities in the areas of biomedical research, worker safety, and environmental protection were correspondingly broadened.

Responsibility for conducting a Biological and Environmental Research (BER) program rests in the OHER of the DOE. Report DOE/ER-0185/4, "Research in Progress: FY 1988" (U. S. Department of Energy 1988) can be consuited for information on legislative history, program objectives, budget levels, and technical content. Central to the present assessment are the broad goals of the BER program from the above report:

The BER program has two main objectives: (1) to develop the knowledge base necessary to identify, understand, and anticipate the longterm health and environmental consequences of energy use and development; and (2) to utilize the Department's unique scientific and technological capabilities to solve major scientific problems in medicine and biology. Meeting the first objective requires an ability to rapidly assess the potential health and environmental consequences of any proposed energy option. This means developing broadly generalizable knowledge and predictive principles, rather than information relevant only to current energy strategies.
In the last decade there have been spectacular developments in laser science and technology and their applications to a host of important problems. In laser spectroscopy alone, there has been a renaissance in the ability to selectively excite and detect atoms and molecules in the gaseous, liquid, and condensed states of matter (Hieftje et a1. 1981; Hurst and Payne 1988; Letokhov 1985, 1987; Lubman 1989; Omenetto 1979; Piepmeier 1986; Radziemski et al. 1987; U.S. DOE 1988). Support for the initial development of one of these techniques--resonance ionization spectroscopy (RIS)--came almost entirely from the Physical and Technology Research Division of OHER. In addition, in carrying out the measurement sciences portion of that program, partial support has been provided for some of the other laser spectroscopies. The purpose for this present study is to provide an independent assessment of the long-term research needs for laser technologies applied to advanced spectroscopic methods. We need to examine the impact that laser spectroscopy could have on the base programs and the special initiatives of OHER. In doing this, we recognize that while the OHER mission has become more complex with the inclusion of chemical by-products of energy production, laser spectroscopies have emerged as possible solutions to the associated measurement problems which have arisen.

This report is organized as follows. Section 2 summarizes the current program of DOE's Office of Health and Environmental Research and provides some remarks on how laser science and technology could beneficially impact most of the research programs. Section 3 provides a brief global perspective on laser technology and attempts to define important trends in the field. Similarly, 
Section 4 provides a global perspective on laser spectroscopy and addresses important trends. Thus, Section 5 focuses on the trends in laser technology and spectroscopy which could impact the OHER mission in significant ways and contains the basis for recommendations made in the executive summary.

For those with limited familiarity with laser technology and laser spectroscopy, reference is made to Appendix I for a list of abbreviations and acronyms.

Appendix II can serve a useful review or tutorial for those who are not deeply involved with laser spectroscopy. Even those familiar with laser spectroscopy and 7 aser technology may find it useful to know precisely what the authors of this document mean by certain specialized terms and expressions. Finally, a note on the style of referencing may be appropriate. Whenever possible a book or a review article is referenced as the preferred citation. However, we frequently found it useful to reference a number of individual papers of recent origin or those which were not conveniently found in the review articles.

\section{REFERENCES}

Hieftje, Gary M., John C. Travis, and Fred E. Lytle, Eds. (1981) LASERS IN CHEMICAL ANALYSIS (The Humana Press, Clifton, $\mathrm{NJ}$ ).
Hurst, G. S. and M. G. Payne (1988) PRINCIPLES AND APPLICATIONS OF RESONANCE IONIZATION SPECTROSCOPY, Adam Hilger, Ltd., Bristol, U.K. and Philadelphia).

Letokhov, V. S., Ed. (1985) LASER ANALYTICAL SPECTROCHEMISTRY (The Adam Hilger Series on Optics and Optoelectronics) (Adam Hilger, Ltd., Bristol, U.K.).

Letokhov, Vladilen S. (1987) LASER PHOTOIONIZATION SPECTROSCOPY (ACademic Press, Inc., Orlando).

Lubman, D. M., Ed. (1989) LASERS AND MASS SPECTROMETRY (Oxford University Press, Oxford), in press.

Omenetto, Nicolo, Ed. (1979) ANALYTICAL LASER SPECTROSCOPY, Vo1. 50 of Chemical Analysis series (John Wiley \& Sons, New York, NY).

Piepmeier, Edward H., Ed. (1986) ANALYTICAL APPLICATIONS OF LASERS, Vol. 87 of Chemical Analysis series (John Wiley \& Sons, New York, NY).

Radziemski, Leon J., Richard W. Solarz, and Jeffrey A. Paisner, Eds. (1987) LASER SPECTROSCOPY AND ITS APPLICATIONS (Marcel Dekker, Inc., New York and Base1).

U. S. Department of Energy Report DOE/ER0185/4 (1988) "Research in Progress: FY 1988," (Office of Scientific and Technical Information). 
2. SUMMARY OF THE OFFICE OF HEALTH AND

ENVIRONMENTAL RESEARCH (OHER) PROGRAMS:

A PERSPECTIVE ON THE USE OF LASER SPECTROSCOPY

\subsection{INTRODUCTION}

The objective of the OHER research program is to obtain knowledge that can be used to minimize the harmful effects of energy production on human health and the environment. Since the research is supported at a fundamental level, numerous benefits follow from applications to other programs.

The research program is categorized by financial allocation in Table 2-1 and program organization in Table 2-2. In order to place future uses of laser spectroscopy in perspective, we follow the outline of the FY 88 Research in Progress report (DOE/ER-01854) in the remainder of this section, addressing areas of need and opportunity without budgetary or organizational restrictions.

\subsection{SOURCE AND DOSE DETERMINATION}

A central focus of OHER Dosimetry Research is the detection and transport of radiation and chemical sources in the environment where human health is at risk. This includes radioactive fallout, toxic or carcinogenic chemicals, and natural environmental sources such as radon. Laser detection methods have seen limited use to date in these areas. However, the need for detectors with much greater selectivity and sensitivity should result in considerable increase in the use of laser spectroscopy.

The OHER Measurement Sciences program supports a number of studies relating to laser spectroscopy for the measurement of the chemical pollutants as atomic or molecular species. The goal of this program is to perform research and advanced developments on analytical systems. Nearly half of the projects in this program area, whose goal is to perform research and advanced developments on analytical systems, utilize laser methods.

\subsection{ENVIRONMENTAL PROCESSES AND EFFECTS}

Comprehensive programs are carried out in the atmospheric, oceanographic, and terrestrial sciences, and integrated into an ecological context. The focus of the Atmospheric Chemistry and Fluid Dynamics program is the physical and chemical fate of pollutants in the atmosphere. Particular attention is given to modeling transport and chemical changes for both flat and mountainous terrains. Wet and dry acid deposits from fossil fuel energy conversion are special concerns. Laser-based instrumentation has been used in a few of these projects. These include laser anemometers to ascertain transport properties of the lower atmosphere; tunable, diode lasers in systems to measure absorption due to the atmospheric deposition of ozone, nitric oxide, and nitric acid; and an airborne IR laser system to measure $\mathrm{SO}_{2}$ by the photoacoustic method.

The Marine Transport and Transformation program emphasizes physical, chemical, and biological factors which affect the transport of materials in the oceans and includes study of the ocean for waste disposal. Natural and artificial radioactive sources serve as primary tools for these studies. These include natural fluorescence measurements, and some limited use of remote sensing with lasers. Laser spectroscopy could play a larger role in studying composition, transport, and oceanic circulation in the future. 
Research Programs

s(Millions)

Total

Laser

Related

Physical and technological research to

38.0

3.3

characterize energy-related emissions humans

may be exposed to and improve measurement and

dosimetry instrumentation, improve under

standing and prediction of atmospheric

transport and diffusion, their chemical

processes and deposition, and to define the

fundamental processes that govern radio-

biological action and biomolecular energetics.

Environmental research to determine the

21.2

0.9

mechanisms which control and influence

total ecosystems and the cycling of energy

by-products through them.

Human health research to quantify risks of

late effects of acute and chronic exposures

25.1

nil

via epidemiologic studies of workers and the

general population.

Health effects research in biological

69.0

0.7

systems to experimentally define and understand dose-response relationships and factors

influencing carcinogenic, mutagenic, and toxicological risks of energy-related exposures.

Nuclear medicine research to develop new radioisotopes, labeled compounds, clinical procedures, and visualization devices for improved diagnoses and treatment of human diseases.

Capital equipment and construction.

11.5

Program direction.

4.0

Total

$\overline{\$ 203.9}$

$\overline{\$ 4.9}$

*Table taken from U. S. Department of Energy Report DOE/ER-0185/4, "Research in Progress: FY 1988," (Office of Scientific and Technical Information, March 1988), p. XV. 
Table 2-2.* Program Organization

\begin{tabular}{|c|c|c|c|}
\hline $\begin{array}{l}\text { Human Health } \\
\text { \& Assessments }\end{array}$ & $\begin{array}{l}\text { Health Effects } \\
\text { Research }\end{array}$ & $\begin{array}{l}\text { Ecological } \\
\text { Research }\end{array}$ & $\begin{array}{c}\text { Physical and } \\
\text { Technological } \\
\text { Research }\end{array}$ \\
\hline Epidemiology & Radiation Biology & $\begin{array}{l}\text { Marine } \\
\text { Research }\end{array}$ & $\begin{array}{c}\text { Atmospheric } \\
\text { Physics and } \\
\text { Chemistry }\end{array}$ \\
\hline \multirow[t]{2}{*}{$\begin{array}{l}\text { Nuclear } \\
\text { Medicine }\end{array}$} & Chemical Toxicology & $\begin{array}{l}\text { Terrestrial } \\
\text { Transport }\end{array}$ & $\begin{array}{l}\text { Measurement } \\
\& \text { Dosimetry }\end{array}$ \\
\hline & $\begin{array}{l}\text { General Life } \\
\text { Sciences }\end{array}$ & $\begin{array}{l}\text { Ecosystem } \\
\text { Response }\end{array}$ & $\begin{array}{c}\text { Radiological } \\
\text { and Chemical } \\
\text { Physics }\end{array}$ \\
\hline
\end{tabular}

*Table taken from U. S. Department of Energy Report DOE/ER-0185/4, "Research in Progress: FY 1988," (Office of Scientific and Technical Information, March 1988), p. xvi .

The Terrestrial Transport and Fate program deals with the movement and possible chemical change. of pollutants such as radon in the earth. Studies using remote fiber spectroscopy (RFS) are of special relevance to this assessment study. The RFS technique can be used for remote chemical detection by direct spectroscopy or by using optrodes -detectors put on the ends of fibers which interact with the chemical to be detected to produce optical signals. This technology could greatly advance the measurement of chemicals in situ and would obviate the need for most conventional drilling techniques.

The Ecosystem Functioning and Response program integrates knowledge from the atmospheric, marine, and earth sciences for a better assessment of the total impact of energy use. Most of the methods used to study ecosystems are conventional: however, there are opportunities for laser spectroscopy in improving the instrumentation used to study the ecosystem. A recentiy published report entitled, "Evaluation of Mid-to-Long Term Basic Research for Environmenta1 Restoration, " DOE/ER-0419, September 1985, addresses research needs for dealing with radioactive, inorganic and organic chemical wastes from the operation, decommissioning, and cleanup at DOE facilities after some 40 years of operation. The plan identifies several high priority needs for improved instrumentation associated with sampling and measurement of containment sources and their transport through the environment. Basic research on methods for remote monitoring and sampling and for more sensitive laboratory assays to reduce sampling costs and health risk to workers was recommended. 


\subsection{HEALTH EFFECTS}

The Radiation Exposure Effects program has four subdivisions and seeks to understand human health risks resulting from exposure to ionizing radiation or energy-related chemicals. The first subdivision, Human Studies, focuses on populations exposed to ionizing radiation from occupational and environmental sources. These include atomic bomb survivors, radium dial painters, uranium miners, and others exposed to radiation. It is conceivable that sensitive methods of laser spectroscopy could be used to measure long-lived isotopes induced by weapons radiation. However, many risks are due to the inhalation or ingestion of isotopes which can emit alpha particles and cause localized damage from within the body. Thus, there is a strong emphasis on radon which is inhaled as a chemically inert gas, but whose daughter products are alpha particle emitters. other problems of a similar nature involve uranium mining or plutonium production. In some of the measurement approaches, specific exposure risks through internal radiation dosimetry are assessed. In these, methods of sensitive detection based on laser spectroscopy could help to determine pathways, transport rates, and localized dose.

The second subdivision, Mammalian Radiobiology, involves studies of the effects on laboratory animals of energy by-products most likely to be encountered by humans. The analytical methods used in these experiments are traditional. However, laser spectroscopy could help to determine the distribution, retention, and dosimetry of internally deposited radionuclides in the laboratory animals.

The third subdivision, Cellular and Molecular Radiobiology, studies efforts to enhance the understanding of late somatic and genetic effects of radiation. Research in radiation genetics and cytogenetics provides essential information on gene and chromosome mutations. Research is aimed also at characterizing the role of molecular damage and its repair in the development of late radiation and chromosomal effects.
The fourth subdivision, Radiological Physics and Chemistry, seeks to understand mechanisms for biological damage. One focus is the interaction of radiation with matter and following the chain of events through the physical, chemical, and biological stages. Microdosimetry is used, and theories of the relative biological effectiveness (RBE) of radiation having different linear energy transfer (LET) values are developed.

The Long-Term Effects of EnergyRelated Chemical Agents program supports the explloration of chemical toxicity problems associated with various energy technologies, including genetic effects and carcinogenesis. Since some chemical pollutants are synergistic in their health effects, their long-term effects are more complex than is their radiation counterpart. Laser spectroscopy could play an increasing role in the near future in studying the interaction of chemicals with genetic material, especially in research that involves studies of DNA.

Under General Life Sciences program, there are five subdivisions. The first, Chemical Physics, Instrumentation, and Iraining, overlaps considerably with Radiological Physics and Chemistry and with the Measurement Sciences program. Some of the instrumentation activities include laser methods for quantitation of electrophoresis, the scanning transmission electron microscope (STEM), neutron scattering, and synchrotron radiation sources.

A second subdivision, Structural Biology, emphasizes innovative methods for biological structure determination. A major program deals with protein structure and conformation, using $X$-ray diffraction for crystalline material and gel filtration for molecules in solution. Studies in the areas of structure, function, and dynamical processes using such advanced techniques as synchrotron radiation, neutron scattering, and STEM are already impressive.

A third subdivision, Molecular Genetics and Gene Requlation, contributes to the knowledge of gene expression, gene function, and cell growth in humans to understand the consequences of 
environmental stresses on man and for developing methods to diagnose and treat diseases. This program contains many new thrusts which rely on advanced technology, such as several projects involving genome sequencing, new methods for rapid preparation of DNA samples for automated sequencing, high resolution STEM used to detect heavy atom clusters, use of resonance absorption, mass spectroscopic identification, and flow cytometry used for genetic analysis and chromosome classification. Human genome mapping is planned, using pulsed field electrophoresis on flow-sorted chromosomes. Los Alamos Mational Laboratory is evaluating new base sequencing approaches such as detection of fluorescing bases and the use of laser desorption followed with mass spectroscopy. A direct imaging method of base sequencing using $X$-ray holography with tagged bases is being investigated by a small business firm (MCR Technology) associated with the University of Illinois at Chicago under an OHER research grant.

The fourth subdivision, DNA Damage and Repair Processes, involves efforts to isolate, clone, and sequence genes that code for enzymes involved in the repair of various types of damage from ionizating radiation. other projects identify biologically important radiation produced lesions in DNA.

The fifth subdivision, Cell Biology, under the General Life Sciences program supports research in basic cell biology to provide basic knowledge of cell function and structure necessary to understand responses to radiation and chemical exposures. Included are studies on chemical carcinogenesis in human cells, cell biology and control of gene expression, regulation of cell membrane support, and an integrated, multilevel study of mechanisms from molecules to man. This, as well as the preceding subdivision, would receive a tremendous boost from methods involving laser spectroscopy which would speed up genome sequencing (see Section 3.4).

\subsection{NUCLEAR MEDICINE APPLICATIONS}

DOE traditionally seeks to derive nuclear medicine benefits from byproducts of nuclear energy production. A major part of this effort is the production of radioactive atoms for use in radiopharmaceuticals. other work involves the preparation of chemical compounds optimized to seek specific human organs, stable compounds for MRI, and special boron compounds for neutron capture therapy.

National laboratories, off-site laboratories, and some small companies have been funded to develop radiopharmaceutical technology. Support for the positron emission tomography (PET) scanner has provided a major diagnostic tool for the study of brain disorders and heart functioning. With support from private industry, threedimensional detectors have been improved to a resolution of about $3 \mathrm{~mm}$.

Separation and enrichment of nuclear medicine isotopes depend on large-scale enrichment facilities such as those at Oak Ridge. Problems arise with the use of these facilities (some of which are obsolete) for producing small quantities of medical isotopes. Enrichment of stable and radioactive isotopes for medical applications may well be economically feasible by the use of laser spectroscopy. Separation of small quantities of isotopes such as sulfur and calcium by use of laser spectroscopy would alleviate the problems encountered with use of large scale facilities. 


\section{STATUS OF RELEVANT LASER TECHNOLOGIES}

\subsection{INTROOUCTION}

In this section we examine the status of those aspects of laser technology which are relevant to laser spectroscopy or to the sensitive measurements of chemical species. Here, we intend to have a broad perspective, leaving for Section 5 the discussion of possible impacts that laser technology could have on the OHER program. Because we identify opportunities for programs in the future, it is necessary in the study of the status of laser technology to identify important trends. Those aspects of laser technology to receive attention are light sources, detectors of laser interactions, imaging techniques, reservoirs of atoms and molecules, and some examples of systems using lasers.

\subsection{SOURCES}

The success of DOE's future programs to apply laser sources to remote sensing, chemical analysis and detection, RIS, isotopic detection and separation, and other important matters depends on an understanding of the present and future development of these sources. This section addresses the current state of the art of lasers and nonlinear techniques for extending the frequency range of lasers to the UV and IR spectral regions. Table 3-1 provides an overview of laser sources, showing typical continuous wave (cw) power, typical pulse energy, typical wavelengths and the primary applications of representative types of lasers. Recent laser technology has brought rapid advances in the areas of efficiency, power, wavelength, tuning range, pulse widths, and peak power levels, which are discussed below.

\subsubsection{Gas and Liquid Lasers}

Helium-Neon. Progress continues to be made in gas laser technology. The helium-neon laser is firmly embedded as a tool for optical alignment, interferometry, bar code reading, siting, and laser velocimetry. Even though diode-laser sources are now available in the red spectral region, helium-neon lasers will continue to serve an important need because of their very long operating lifetime and compact design. Argon-Ion. Argon-ion laser technology has matured. Today, large frame argon-ion lasers operate at up to $20 \mathrm{~W}$ of $\mathrm{cW}$ output power at approximately $40 \mathrm{~kW}$ of input power. The plasma-tube lifetime has been extended to a few thousand hours by the use of segmented ceramic-metal construction methods. Wavelength operation now extends into the UV region with the use of special mirrors for the laser resonator. Argon-ion lasers find wide application in pumping dye lasers for tunable laser output. Recently, argon-ion lasers have been used to pump the tunable titanium-sapphire laser, which tunes over a 780 to $1000 \mathrm{~nm}$ wavelength region and operates much like a $\mathrm{CW}$ dye laser. The operational cost of an argon-ion laser remains high, both in terms of electrical power cost and plasma-tube replacement. For this reason, large-frame argon-ion lasers are used in research and not in production applications. However, air-cooled, argon-ion lasers at the $10 \mathrm{~mW}$ output power level are used for production, for example, in color separation processes. The argon-ion laser technology is already mature and is not expected to change appreciably in the near term. 
Table 3-1. Summary of Laser Sources

\begin{tabular}{|c|c|c|c|c|}
\hline TYPE & $\begin{array}{l}\text { TYPICAL } \\
\text { CW POWER }\end{array}$ & $\begin{array}{l}\text { TYPICAL } \\
\text { PULSE } \\
\text { ENERGY }\end{array}$ & $\begin{array}{l}\text { TYPICAL } \\
\text { WAVELENGTH } \\
\mathrm{nm}\end{array}$ & $\begin{array}{l}\text { PRIMARY } \\
\text { APPLICATION }\end{array}$ \\
\hline
\end{tabular}

$\mathrm{GaS}$
$\mathrm{He}-\mathrm{Ne}$$\quad 10 \mathrm{~mW}$

633

barcode reader

surveying, interferometry

GaS Ion $20 \mathrm{~W} \quad 500$

pump for ringdye laser and titanium sapphire, high resolution spectroscopy

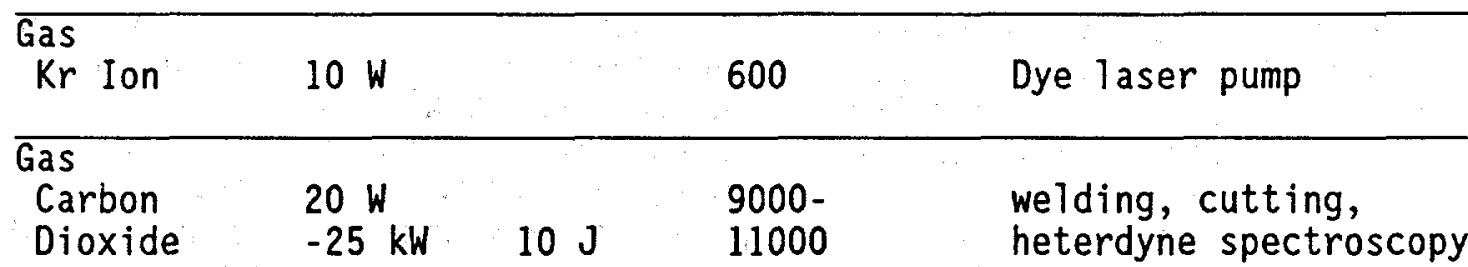

Easimer $1 \mathrm{~J}$

ArF 193

$\mathrm{KrF} \quad 248$

$\mathrm{XeCl} \quad 308$

$\mathrm{XeF} \quad 531$

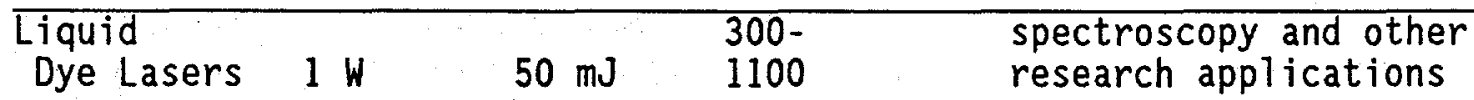

\begin{tabular}{lr}
\hline Solid State \\
Diode Lasers $1 \mathrm{~mW}$ & $3000-$ \\
Various & 30000 \\
Lead &
\end{tabular}

Salts

800 telecommunications, pump for Nd:Yag lasers

$\begin{array}{lll}\text { Nd:YAG } 20 \mathrm{~W} & 1 \mathrm{~J} \quad 1064 & \begin{array}{l}\text { laser ranging, welding, } \\ \text { surgery and pumps for } \\ \text { dye lasers }\end{array}\end{array}$

\begin{tabular}{|c|c|c|c|}
\hline Alexandrite $300 \mathrm{~W}$ & $300 \mathrm{~mJ}$ & $\begin{array}{l}\text { Tunable } \\
700- \\
800\end{array}$ & $\begin{array}{l}\text { materials processing, } \\
\text { spectroscopy, } \\
\text { remote sensing }\end{array}$ \\
\hline
\end{tabular}

\begin{tabular}{|c|c|c|c|c|}
\hline $\begin{array}{l}\text { Titanium } \\
\text { Sapphire }\end{array}$ & $3 W$ & $200 \mathrm{~mJ}$ & $\begin{array}{l}\text { Tunable } \\
780- \\
1000\end{array}$ & $\begin{array}{l}\text { potential dye laser } \\
\text { replacement }\end{array}$ \\
\hline
\end{tabular}


Carbon-Dioxide. The $10 \mu \mathrm{m}$ wavelength carbon-dioxide laser technology has continued to advance in power, wavelength control, coherence, efficiency, and pulse width. The high-power, carbon-dioxide lasers now operate at greater than $25 \mathrm{~kW}$ for industrial applications. The overall operating efficiency is approximately $5 \%$. The lasers are now fully developed commercial sources that are useful tools on the factory floor. Lower-power, carbon-dioxide lasers are used for medical applications and for research. Power levels of $20 \mathrm{~W}$ to $100 \mathrm{~W}$ are common from low-pressure discharge tubes, 1 meter in length. These lasers offer wavelength selection in the 9 to $10 \mu \mathrm{m}$ region with the use of an internal grating. With feedback control, linewidths of less than $1 \mathrm{kHz}$ have been demonstrated.

Carbon-dioxide lasers making use of wave guides are now being manufactured by a number of vendors. These polarized, single-mode lasers operate at up to $20 \mathrm{~W}$ of power for a length of less than 1 meter. Their compact size and guided wave operation make these lasers useful for laboratory scale applications as well as for coherent-7aser radar. The carbondioxide, coherent-laser radar systems are now well developed and are being used for applications from wind velocity sensing to coherent-laser radar for military applications.

Excimer. Following the discovery of laser action on dimer and excimer transitions of $\mathrm{KrF}, \mathrm{XeF}$, and ArF in the early 1970's, excimer-iaser technology has undergone a rapid development. These laser sources operate in a pulsed mode with a large aperture that is typically centimeters in dimension. They operate with pulse durations of tens of nanoseconds with pulse energies up to $1 \mathrm{~J} / \mathrm{pulse}$ for commercial laser sources. Pulse repetition rates exceeding $200 \mathrm{~Hz}$ with average power at the $1 \mathrm{~kW}$ level have been reported.

Excimer lasers are used in research to pump tunable dye lasers and are used directly for excitation of atoms and molecules. Recent applications of excimer lasers include UV lithography and UV circuit repair. The laser sources require flowing gas and high-voltage, pulsed discharge for heat removal and excitation. The lasers operate at up to $3 \%$ efficiency for 7 ifetimes of 1000 hours before discharge electrodes need exchanging. Special handling of fluoride gases is necessary for prolonged operation. However, excimer lasers have become the standard source for UV radiation at $193 \mathrm{~nm}, 248 \mathrm{~nm}$, and $308 \mathrm{~nm}$. Liquid Lasers. The dye laser represents the most important class of liquid lasers. Dye lasers offer wavelength tunability from the near IR near $1000 \mathrm{~nm}$ across the visible to the UV near $350 \mathrm{~nm}$. Dye lasers can operate $\mathrm{CW}$ when pumped by an argon-ion laser source or pulsed when pumped by an excimer laser or a Q-switched, Nd:YAG Taser source. Further, dye lasers can be flashlamp pumped to produce pulses with durations of about 1 microsecond at the energy level of about $1 \mathrm{~J}$. Lamp-pumped dye lasers are finding applications in medicine where the 1 us pulse, low peakpower output is an advantage for optical fiber delivery of the radiation.

The $\mathrm{Cw}$ dye laser pumped by an argonion laser is a very widely used tunable source of laser radiation in the research environment. Dye lasers operate at over $1 \mathrm{~W}$ of optical output power. Wavelength control is achieved using intracavity elements such that dye lasers can continuously tune over a wide wavelength range. The $\mathrm{Cw}$ dye laser can al so be tuned continuously over a $30 \mathrm{GHz}$ region with a linewidth of $1 \mathrm{MHz}$. With feedback control, the dye laser 7 inewidth has been reduced to less than $1 \mathrm{~Hz}$.

Dye lasers, pumped either with the excimer laser or a Q-switched, Nd:YAG laser, can be pulsed at repetition rates from $10 \mathrm{~Hz}$ to greater than $100 \mathrm{~Hz}$. These dye lasers operate with 10 to 100 ns pulse widths at peak powers greater than $10 \mathrm{~mW}$. The tunable, high peak-power, radiation can be frequency extended by nonlinear techniques into the UV and the near IR. The mode-locked, $C w$ dye laser with proper group velocity control has operated at less than 5 fs pulse widths. The dye laser thus provides the shortest optical pulses yet generated. It is expected that the dye laser will remain the laser source of choice for research applications. Dye lasers have not been 
chosen for many production applications because the dye must be changed periodically. The recently introduced titanium-sapphire laser will eventually impact the use of dye lasers.

\subsubsection{Solid-State Lasers}

Diode Lasers. Diode Tasers, other solid-state lasers, and nonlinear optical devices were first demonstrated at the dawn of the laser age over 25 years ago. Today, it is the convergence of these technologies that is rapidly revolutionizing the laser source capability. For example, diode-laser sources are now being shipped in commercial products at over 12 million units per year. Diode lasers operate at over $1 \mathrm{~W}$ of $\mathrm{CW}$ power per facet with more than $75 \mathrm{~W}$ of $\mathrm{CW}$ power per diode-laser array. The quantum well type of diode lasers operate with an internal differential quantum-efficiency of greater than $95 \%$ and with an overall electrical efficiency of greater than $50 \%$. Laser 1 ifetimes have been extended to tens of thousands of hours and even up to 100,000 hours for diode lasers used in fiber-optic communications.

Finally, and of crucial importance for the future, diode-laser prices are now following the semiconductor device historical pricing trends and are falling a factor of four per year per watt of output power. Indeed, the goal of semiconductor manufacturers in Japan and in the United States is to produce $1 \mathrm{~W} \mathrm{cW}$ diode lasers for less than $\$ 10$ each by 1992.

The diode-laser technology is also extending available wavelengths. For example, diode lasers are now operating at visible wavelengths of less than $650 \mathrm{~nm}$ at $3 \mathrm{~mW}$ of output power. Pulse operation has been obtained into the yellow region. Blue emitters require IIVI compounds with pn junction capability, thus the prospects for an electrically pumped diode laser in the blue appears to be at least 10 years away. However, electron-beam pumped and optically pumped diode lasers are being studied as are the materials parameters necessary to construct a II-VI compound laser for direct blue generation.

In an alternative approach, diode lasers have been efficiently frequencydoubled to generate $410 \mathrm{~nm}$ radiation. Two approaches to the doubling have been demonstrated: (a) resonant external cavity doubling in potassium-niobate crystals and (b) direct doubling in a channel-guide, Tithium-niobate doubler that used quasi-phasematching to achieve the $410 \mathrm{~nm}$ output. The possibility now exists for doubling diode lasers directly to efficiently generate blue radiation by using inexpensive, lithographically defined waveguide doublers in 1 ithium niobate.

The average output power of diode lasers is increasing by a factor of two per device each year. This year a commercial product was introduced that generates $10 \mathrm{~W}$ of $\mathrm{CW}$ output power. A $50 \mathrm{~W}, \mathrm{CW}$-output power device has been demonstrated in the laboratory. The output power is expected to exceed $30 \mathrm{~W} / \mathrm{mm}$ of diode-1aser length. There has also been progress on two-dimensional diode-laser arrays. Recently, faceemitting diode lasers have been demonstrated that are expected to achieve greater than $40 \%$ operating efficiency. Two-dimensional arrays of diode lasers may be expected to emit greater than 1000 $W / \mathrm{cm}^{2}$ of optical power.

Applications of diode lasers include pumping of solid-state lasers, reading and writing compact disks for digital and for analog information, bar code readers, optical radar, electro-optical sampling of high-speed circuits, and chip-to-chip communication. Diode lasers are the most efficient source of coherent or incoherent optical radiation. Applications of frequency converted diode lasers include red, blue, and green displays for projection of high definition television images and for color separation.

Other Solid-State Lasers. During the past five years, solid-state lasers have undergone a rapid evolution in power, wavelength diversity, tunability, and efficiency. The ruby laser, the Nd:YAG laser, and the Nd:Glass laser are all well developed technologies. The flashlamp-pumped lasers now generate very 
high peak power in Q-switched pulses. For example, the commercial unstableresonator, Nd:YAG laser introduced in 1976 uses flashlamp pumping to produce $1 \mathrm{~J} / \mathrm{pul}$ se at up to $30 \mathrm{~Hz}$ repetition rate in a $10 \mathrm{~ns}, \mathrm{Q}$-switched pulse or $100 \mathrm{MW}$ of peak power. This laser can be efficiently frequency-doubled to the green and converted further to $355 \mathrm{~nm}$ and $266 \mathrm{~nm}$ in the UV. The frequency-doubled Nd:YAG laser is useful for pumping dye lasers as mentioned above. Recent progress in this technology has led to injection seeding for single-axial mode control. Single frequency operation by injection seeding leads to very stable peak-to-peak power output which is important for frequency-doubling applications such as spectroscopy or materials processing.

In the late $1970^{\prime} \mathrm{s}$ the tunable, alexandrite laser was introduced. Alexandrite is a crystal doped with chromium ion that provides tunable radiation in the 700 to $800 \mathrm{~nm}$ region. The laser can be flashlamp pumped, CW arc-lamp pumped, or pumped with other lasers. To date, alexandrite lasers have been developed for research applications, particularly for remote sensing. Recent work has led to alexandrite lasers with output power levels in excess of $300 \mathrm{~W}$ which operate at $3 \%$ efficiency and are useful for industrial applications.

The tunable, alexandrite laser led to further investigations of tunable, solidstate lasers. In particular, the tunable, titanium-sapphire laser can be laser pumped like a dye laser and operate over a wide tuning range, from 700 to $1000 \mathrm{~nm}$. Titanium-sapphire lasers have been introduced as commercial products for tunable sources complementary dye lasers in the near IR region and producing $1 \mathrm{~W}$ of $\mathrm{cW}$ radiation.

The importance of high average-power has led to the development of Nd:YAG laser sources for industrial applications, producing kilowatts of average power. To date, companies have introduced multiple-rod lasers that generate more than $1 \mathrm{~kW}$ of average output power in a multimode, nondiffractionTimited beam. There is work in progress to generate high average-power by using slab geometry lasers of both $\mathrm{Nd}$ :YAG and
Nd:Glass. Slab lasers of the YAG variety have operated at greater than $400 \mathrm{~W}$ of average power with $3 \%$ efficiency. Slab lasers using glass have operated at $30 \mathrm{~J} /$ pulse at $1 \mathrm{~Hz}$ or $10 \mathrm{~J} /$ pulse at $5 \mathrm{~Hz}$. Even higher average-power appears possible, using the moving-slab laser concept to dissipate the heat over a wide area. A moving-slab, glass laser with $390 \mathrm{~W}$ of average power has been demonstrated in Japan at Hoya, Inc.

Flashlamp-pumped, solid-state lasers have efficiencies in the 3\% to $5 \%$ range. Q-switched, or mode-locked, solid-state lasers for operation at high peak-power levels are $1 \%$ efficient. A leap in efficiency has been obtained by replacing the flashlamp with diode lasers as pumps in solid-state laser systems.

Diode-Pumped, Solid-State Lasers. By pumping solid-state lasers with diode lasers, the power of many diode lasers can be summed and extracted in a diffraction-limited beam of a single frequency. Further, the ability of ions doped into a solid to store optical energy for many milliseconds allows the $\mathrm{CW}$ diode-laser power to be extracted in high peak-power, Q-switched or mode locked pulses with pulse widths of nanoseconds to picoseconds. Diodepumped, solid-state lasers were first explored in 1963 shortly after the invention of the diode laser. Today, diode-pumped, solid-state lasers are operating with Nd:YAG, Nd:YLF, Nd:Glass, and $H_{0}: Y A G$ at 2.1 microns, and in $E r: Y A G$ at 1.55 microns and 2.9 microns. Diodepumped, nonplanar-ring, Nd:YAG lasers have operated at a single frequency with linewidths that are less than $2.9 \mathrm{~Hz}$ and subhertz linewidths may be possible soon. Diode-pumped, solid-state lasers are $Q$ switched with kilowatt peak power levels, are mode locked with less than 10 ps pulse widths, and are operated $\mathrm{Cw}$. Programs now underway promise to raise the average power level to greater than $100 \mathrm{~W}$ by 1992, and have been considered for producing megawatts of average power. Recentiy, and of critical importance to many applications, diode-pumped, solid-state lasers have now operated at greater than $20 \%$ electrical efficiency.

The technology is advancing rapidly

in Europe, Japan, and in the United 
States. The recognition of the importance of this new solid-state laser technology is illustrated by the fact that more than seven startup companies have been formed in the past two years to exploit diode-pumped, solid-state-laser technology. However, the United States is in danger of losing the lead in a technology that was pioneered in this country because of the lack of investment in the low-cost production and manufacture of diode lasers and diodelaser arrays for potential applications to commercial markets.

The high efficiency of solid-state lasers when pumped by diode-laser arrays opens many application areas that were heretofore impractical to consider. Among them are laser-driven chemistry, isotope separation, remote sensing, coherent laser-radar, high-definition television (HDTV) image projection, UV and X-ray lithography, and optically driven particle accelerators. Some industrial applications that require compact, highly efficient, high averagepower laser sources have also been considered.

\subsubsection{Free-Electron Lasers}

Free-electron lasers make use of electron accelerators and other specialized components such as magnets (called wiggler magnets) to produce magnetic fields with spatial periodicity. The free-electron laser is usually viewed as a user-group facility, since it is too expensive to be widely duplicated. The first free-electron laser, at Stanford University, has been replaced with a new device. Since 1985 about 10 devices have been operated at wavelengths from the millimeter region to the visible (Brau 1987).

In these lasers, electron beams are passed through wiggler magnets at velocities close to the speed of light. Thus, the electrons execute a wiggly motion along the path through the magnetic field and radiate at the frequency of the oscillation. The wavelength of the radiation is Doppler shifted to shorter wavelengths and is given by $\lambda s / \gamma^{2}$, where $\lambda s$ is the wiggler period, (i.e., the distance to complete one cycle of the spatially varying magnetic field), and $\gamma$ is the ratio of the electron energy to the electron rest energy $(0.511 \mathrm{MeV})$. Thus, the wavelength of the free-electron laser is controlled by the length $\lambda s$, determined by the magnetic field, and the energy of the electron accelerator. With electron energies of $100 \mathrm{MeV}$ it is practical to produce wavelengths in the visible region. For a fixed wiggler period, a tuning range of a factor of 2 or 3 is achieved by changing the electron energy. Although the laser gain decreases with increasing frequency, this can be largely offset by producing higher intensity electron beams of very low spread in emission, as demonstrated by the development of a photoelectric injector at Los Alamos National Laboratory. New techniques are also being studied for producing much shorter wiggler periods permitted by the higher quality electron beams. Acceleration techniques based on lasers could permit much shorter accelerators than does the current microwave technology. These developments, together with the new mirror technology, may permit widely tunable, compact, and efficient freeelectron lasers for wavelengths as short as $10 \mathrm{~nm}$ and as long as millimeters, with very high peak and average power.

Free-electron lasers producing high average-power at short wavelengths would be useful in computer chip fabrication, in solid-state research, in biological and medical applications, and in chemical processing. Their intensity far exceeds that of synchrotrons within their frequency operating range.

\subsubsection{X-Ray Lasers}

The promise of coherent sources of high intensity radiation in the $X$-ray region as a tool for condensed matter research, (particularly in the field of biology and medicine), has led to continuing efforts to produce $X$-ray lasers. These lasers require extremely high pumping rates to generate the necessary population inversion. For 
instance, it has been shown that a $1 \mathrm{kV}$ laser requires a deposition rate $>10^{14} \mathrm{~W} / \mathrm{cm}^{3}$, and it is only recently that the appropriate power densities have become available, using high power lasers as the pump. Two general techniques have been proposed, and both have been successfully demonstrated.

The first of these, originally demonstrated at Lawrence Livermore National Laboratory, involves the laser heating of a cylindrical volume with a cross section of several tens of micrometers and several centimeters in length. This requires a sufficiently high temperature to produce neon-like ions of moderate atomic weight, such as selenium. Electrons in the plasma are allowed to cool and recombine to fill the inner shell vacancies at a sufficient rate to produce gain and subsequent lasing. Since this process competes against black-body radiation, as the plasma is optically thick, it is quite inefficient $\left(-10^{-10}\right)$, but the very high power and energy used produces an $X$-ray beam of high intensity. Because of the increasingly strong competition with other modes of energy loss, it appears doubtful that wavelengths $<3 \mathrm{~nm}(400 \mathrm{eV})$ can be produced by this technique, although this wavelength would work in the water window. However, radiation damage to biological material appears to be a severe problem.

A second approach for producing an $X$-ray laser has been demonstrated by a group at the University of Illinois at Chicago, in which the pump energy is coupled only to the electrons, leaving the ions cold. An extremely highintensity UV beam pulsed in femtoseconds produces inner shell excitation of multiply excited states by an efficient multiphoton coherent process. This results in highly selective transitions to either low lying or ground state levels of the ions, ensuring inverted populations. The resulting high gain of the stimulated emission produces a traveling-wave laser which follows the pumping pulse due to the short emission time of the transitions. A number of lasing transitions between 50 and $100 \mathrm{~nm}$ have been observed in argon, krypton, and xenon; and scaling laws have been derived which predict that the present laser pump should produce wavelengths as short as $1 \mathrm{~nm}$. A self-channelling mechanism is predicted which should produce $X$-ray laser beams a few micrometers in diameter.

It is important to note that the $X$-ray laser mechanism postulated to explain these experimental results would result in pulses of $100 \mathrm{fs}$ duration with sufficient intensity to produce $X$-ray holograms of living material in its hydrated form without distortion. The target would be completely destroyed by both thermal and radiation damage effects, but only after the information had been obtained. Other laser processes with longer pulses could not avoid these destructive effects.

\subsubsection{Nonl inear Frequency Extension}

The potential applications of lasers, even with average power in the kilowatts region, is limited unless the radiation can be shifted by nonlinear techniques to a frequency region of interest. Some recent demonstrations have been made of nonlinear optical techniques which allow wavelength extension of the diode-pumped, solidstate lasers which are efficient at the milliwatt-power levels. It has long been recognized that lasers with high peak power can be efficiently converted to other wavelengths by second harmonic generation, sum and difference frequency generation, and optical parametric oscillation. Pulsed lasers with high peak power operating in the near-IR region can be frequency-doubled, -tripled, and-quadrupled with greater than $50 \%$ conversion efficiency in potassium di-hydrogen phosphate (KDP).

What is new is the demonstration in the laboratory of efficient harmonic generation at the milliwatt-level with $\mathrm{cw}$ laser sources. By taking advantage of the frequency stability and single frequency operation of the diode-pumped, solid-state laser, $\mathrm{cw}$ doubling using external resonance techniques has been achieved at greater than $56 \%$ conversion efficiency. For example, $52 \mathrm{~mW}$ of $\mathrm{cW}$ at a $1064 \mathrm{~nm}$ output from a diode-pumped, 
Nd:YAG, ring laser has been converted to $30 \mathrm{~mW}$ of $\mathrm{CW}$ green radiation with an overall conversion efficiency of $56 \%$. The internal conversion efficiency within the $12 \mathrm{~mm}$ long, 1ithium-niobate crystal was $72 \%$. Improvements in the coatings applied to the crystals will likely raise the conversion efficiency to greater than $85 \%$ in the near future.

For efficient nonlinear conversion, the phase velocity of the fundamental and harmonic waves must be matched. Traditionally, this was accomplished by using the birefringence of the nonlinear crystal to offset dispersion. Another approach is to reverse the sign of the nonlinear coefficient each time the phase of the fundamental and second harmonic wave is reversed; accomplished by reversing the sign of the polarization in a ferroelectric crystal such as lithium niobate. Recent results have led to the first demonstration of this periodic poled phasematching for harmonic generation of blue light at $420 \mathrm{~nm}$ in lithium niobate. This technology allows nonlinear processes to be optimized across the entire transparency range of lithium niobate from $400 \mathrm{~nm}$ to beyond $4000 \mathrm{~nm}$ in the IR. For example, a diode laser can be frequency doubled in a lithium-niobate waveguide by using periodic poling to phasematch the interaction. This creates the possibility of using lithographic, planar-processing techniques to build frequency doubling crystals on planar substrates in large quantities and at low cost.

Tunable Parametric 0scillators. The optical parametric oscillator (OPO) converts high frequency radiation into tunable low frequency radiation. For example, green radiation from a doubled Nd:YAG 1aser source can be used to pump a parametric oscillator in lithium niobate to generate tunable output from the 680 to $4000 \mathrm{~nm}$ spectral range. Previous work in parametric oscillators has used pulsed-laser sources for pumping. Recent work has shown that the parametric oscillators can operate $\mathrm{cw}$ with a $910 \mathrm{~mW}$ threshold. When pumped at $20 \mathrm{~mW}$, the $\mathrm{CW}$ lithium-niobate parametric oscillator operated at $80 \%$ conversion efficiency with less than a $20 \mathrm{kHz}$ linewidth.

Parametric oscillators have also been operated in a sync-pumped, modelocked manner with tunable output at 300 fs pulse lengths. Ultraviolet radiation from the third and fourth harmonics of a Q-switched Nd:YAG laser has been used to pump barium borate as OPO's. Using the crystal beta-bariumborate, (first grown in Germany but developed in China), a $355 \mathrm{~nm}$ pumped parametric oscillator has been continuously tuned over a wide range of wavelengths. This widely tunable OPO operated at $30 \mathrm{~Hz}$ repetition rate at over $35 \%$ optical conversion efficiency at greater than $140 \mathrm{~mW}$ of average output power. The tuning range was achieved by rotating the crystal angle by just a few degrees. Upon injection seeding, the parametric oscillator operated at a single frequency.

In summary, $O P O^{\prime} s$ offer the potential for all solid-state, tunable sources with wavelength tuning from the UV to the IR. The improvement in nonlinear materials, coupled with the improvement in laser-pump sources, has led to increased interest in parametric oscillators as solid-state alternatives to the dye laser.

High Average-Power, Nonlinear Processes. High average-power, nonl inear optical conversion is now being investigated. Recent results show that the expected doubling efficiency to green and to the UV is greater than $85 \%$ in each step of the process. For example, second-harmonic generation of a $10 \mathrm{~W}, \mathrm{cw}$, Nd:YAG laser using external resonant doubling in barium borate should give $8 \mathrm{~W}$ of green output. A second barium-borate crystal should allow efficient generation of $266 \mathrm{~nm}$. Thus, based on the theoretical findings, the harmonic generation processes are no longer the limiting factor in producing efficient radiation at wavelengths extending into the UV.

Earlier work has shown that the barium-borate crystal can be used to frequency double even when the average power levels exceed $100 \mathrm{~W}$ without special precautions. There is an opportunity, 
with future research, to extend harmonic generation to the average-power level of kilowatts using barium borate. This, in turn, opens the possibility for development of an all solid-state source of tunable, $\mathrm{cw}$, coherent radiation with greater than $20 \%$ electrical efficiency. Such a laser source could be used to improve the separation rate of isotopes and at lower cost. Further, such a laser source could offer the potential of extending laser processing to other chemical applications since the cost per photon is less than the most efficient incoherent source of radiation--the mercury discharge.

Picosecond and Femtosecond Laser Sources. The 1970's were characterized by the picosecond-time scale. The $1980^{\prime} \mathrm{s}$ ushered in the femtosecond-time scale. To date, the shortest optical pulse generated is approximately 5 optical cycles in length. The pulse was generated in a dye laser by using methods to cancel group velocity dispersion.

In recent work, short pulses have been generated in Nd:Glass, Nd:YAG, and Nd:YLF when these were pumped with diode lasers. The pulse lengths for $\mathrm{cw}$ operation under diode-laser pumping are now 6 ps. However, by using pulse chirping in an optical fiber followed by pulse compression using a grating pair, the pulse length can be reduced to the 10 to $100 \mathrm{fs}$ region. This source of short optical pulses requires no water cooling and can be operated as a simple plug-in to existing sampling scopes. These picosecond and femtosecond, all solid-state, lasers will find applications in electro-optic sampling, chip-to-chip communications, and perhaps in biology for measurements of 1 ifetimes of excited states.

Recent work has also shown that it is possible to generate pulses with terrawatt peak power by using pulse expansion and amplification, followed by pulse compression. A group at the University of Rochester has obtained pulses with terrawatt peak power, i.e., $1 \mathrm{~J}$ in a picosecond pulse, by the use of pulse compression from 1000 ps to 1 ps. This technique is important because it allows efficient extraction of stored energy from a solid-state laser without damage arising from nonlinear selffocusing due to self-phase modulation.

\subsection{DETECTORS FOR LASER SPECTROSCOPY}

\subsubsection{Photon Detectors}

There is a growing need in many fields of research for photon detectors that are faster, have a broader spectral range, and are more sensitive than currently available detectors. Research applications include monitoring fastpulsed lasers and characterization of high-speed optical components such as modulators, detectors for atomic and molecular spectroscopy, and integrated optical detectors for the new generation of compact flow cytometers.

Solid-state photon detectors offer fast response times and small size. At lower frequencies, the most sensitive photon detectors are avalanche photodiodes because of the internal gain of these devices. (Bowers and Burrus 1987). At higher frequencies, a $p-i-n$ photodiode followed by a field effect transistor (FET) amplifier is a better choice. Photoconductive detectors have the edge when response times of less than 1 ps are required, although the detector sensitivity is greatly reduced.

Solid-state photon detectors can be incorporated directly into integrated optical assemblies. Monolithically integrated amplifiers and detectors have been fabricated using standard ionimplanted technology (Wojtczuk et al. 1987). A photoconductor version of the device has a relatively high gain of 27 and a bandwidth of $25 \mathrm{MHz}$. A photodiode version has a maximum sensitivity at $800 \mathrm{~nm}$, making it potentially useful in flow cytometry for detecting fluorescence. Shottky-barrier photodetectors with an interdigital electrode configuration have been fabricated on a silicon-on-sapphire (SOS) substrate (Bruce et al. 1987). The impulse response time is less than $30 \mathrm{ps.}$ The wavelength range is from the IR to the UV. This design provides efficient coupling of light from an optical 
waveguide into the photodetector fabricated on the same substrate.

Measurements of the fluorescence lifetimes of biological molecules require photon detectors with high quantum efficiency, high gain, fast time response, and the ability to do photon counting. Microchannel plate photomultipliers (MCP-PMT) have been developed recently with these characteristics (Kume et a1. 1988). A microchannel plate (MCP) is a secondary electron multiplier constructed from millions of glass capillaries fused together and cut to form a thin disk. The MCP is placed between the photocathode and the anode of the PMT and has a potential across it of perhaps $3000 \mathrm{~V}$. An electron striking the wall of one of the microchannels produces secondary electrons which give rise to a cascade of further secondary electrons. The gain of a single stage MCP-PMT can be as high as $10^{4}$. With an MCP-PMT having $6 \mu \mathrm{m}$ diameter capillaries, it is possible to measure fluorescence decay time constants as small as 6 ps with a precision of 3 ps. Further development of these devices can be expected to result in faster response times and higher gains.

Silicon-intensified target

vidicon photon detectors have found numerous photon detectors have found spectroscopy (0lesik and Walters 1984). An image intensifier multiplies and focuses photoelectrons onto a silicon target. The target is an array of $500 \times 500$ capacitors that store signals related to the number of photoelectrons striking each picture element (pixel). The pixels are read out by a scanning electron beam. Time-resolved emission spectra have been obtained by suitably programming the scanning readout. The two-dimensional capability of the vidicon has been used for fluorescence measurements by dispersing the excitation light along one dimension and the emission light along the other.

Sodium salicylate is commonly used as a photon detector in the wavelength range from $20 \mathrm{~nm}$ to $360 \mathrm{~nm}$. The sodium salicylate, which is usually sprayed directly onto the outside of the photocathode, converts the UV photons into photons with an average wavelength of $443 \mathrm{~nm}$. This longer wavelength radiation is easily detected with a bluesensitive photocathode material.

During the very early years of Raman spectroscopy development, most spectral data were detected by photographic emulsions. With the advent of scanning spectrometers, photoelectric detection, based upon a photomultiplier fabricated from a photoemissive cathode and a current amplifier within a glass envelope, became the usual basis for recording Raman data. The most common photocathodes are the bialkali systems as, for example, (Cs) $\mathrm{Na}_{2} \mathrm{KSb}$. Ramanscattered radiation generally involves low light levels, producing weak electrical currents from these surfaces. When the current is of the order of $10^{-11}$ to $10^{-13}$ amps, photomultipliers are conventionally used in pulse or photon counting modes. For photocurrents in the $10^{-9}$ to $10^{-11}$ amp range, direct current detection methods may be applied. For systems with high background radiation, phase-sensitive detection techniques have proven useful. The low signal levels encountered in Raman studies can also be recorded with a variety of intensified optical multichannel array detectors. These detectors can be cooled to about $-40^{\circ} \mathrm{C}$ to reduce noise levels. The chargecoupled device (CCD) arrays are being applied to Raman spectroscopic studies. Since a CCD may. contain an array of 400 $x 600$ pixels, large amounts of data can be generated with a single exposure of the chip to the Raman radiation. The two-dimensionality of these low-noise CCD detectors will be useful for obtaining both spectral and spatial information.

\subsubsection{Ionization Detectors}

Sensitive detectors of ions (electrical fragments of matter) have been available since the turn of the 20th century. The first electrical counter (the precursor to the proportional counter) was developed by Rutherford and Geiger in 1908. In the succeeding 40 years, this detector was refined by Curran et al. (1949) to such a degree that single electrons could be detected. 
A closely related detector, the GeigerMueller counter, can also count individual electrons, but, unlike the proportional counter, it cannot distinguish one electron from several or a million created inside its gaseous medium at the same instant in time.

The proportional counter was combined with lasers to count single atoms. This was made possible by the development of RIS by Hurst et al. (1975). RIS is a process (see Section 4.5) in which a laser can be "tuned to an atom" so that each atom of the selected type will give up one of its orbiting electrons when subjected to a single pulse of a laser. This illustrates the enormous analytical sensitivity for counting atoms when laser spectroscopy is combined with ionization detectors.

In addition to the proportional and the Geiger-Mueller counters, there are other methods for counting single electrons. In an evacuated enclosure, single electrons can be accelerated to strike the first surface of an "electron multiplier" tube where an electron cascade is initiated. The "channeltron" is another detector in which electrons are amplified sufficiently to record one initial electron.

often it is desirable to do a mass analysis of the atom (or a molecule) which has been ionized with a laser. In this case, it is necessary to detect the positive ion. Fortunately, the positive ions can be accelerated into a vacuum to a modest energy so that on impact with a converter plate, several electrons are emitted. Thus, single electron detectors can be used to detect single ions. By using a combination of lasers with mass spectrometers (see Sections 3.6.1 and $4.5)$, it is possible to achieve nearly single-atom sensitivity, even when both the type of atom and its mass are selected.

\subsection{IMAGING}

A number of recent developments are providing a basis for important contributions of video imaging in areas of interest to OHER. Computer development with increasing capability in graphics and in high-speed processing of vast amounts of data with sophisticated software, together with the steady decrease in computing cost, makes possible the processing and display of a wide variety of sensor data as video images. Internal relationships, structure, and meaning can be recognized and analyzed more easily in the video format. Excellent examples are computeraided, $X$-ray tomography and nuclearmagnetic-resonance scans, both of which can reveal details of the structure of the soft tissues and organs of the human body. New detectors such as the CCD or charge injected devices (CID) record twodimensional, digital images with much higher sensitivity ( -1 photon) and dynamic range or contrast $\left(-10^{5}\right)$ than does film but with somewhat lower resolution $(-2007$ ines $/ \mathrm{mm})$. New video-imaging devices such as flat-screen, full-color, liquid-crystal displays are also appearing. These promise much lower cost, improved convenience, and greater reliability than do cathode-ray tubes.

Sophisticated computer codes permit the two- and three-dimensional and time development of complex data fields such as temperature, density, composition, and structure in real time. Image enhancement can filter, increase contrast, or reduce noise to bring details out of an apparently featureless background in many situations. Computer codes also permit the construction of colored video images of models or simulations in two and three dimensions which can be rotated, stretched, or modified as directed or under the influence of a superimposed environment.

\subsection{RESERVOIRS}

\subsubsection{Introduction}

A key development for trace detection of atoms and molecules by laser radiation is the design of new sources for vaporization into the gas phase. The problems are very different, depending upon whether the sample is atomic or molecular. In the case of atomic reservoirs, the main problem is to 
vaporize a refractory sample (either as a solid or dissolved in a liquid) into free atoms in the gaseous state. The sample must then be transferred into the probing volume of the light source and remain in that volume for a period of time long enough to assure efficient detection by laser-based spectroscopic methods. In the case of molecular reservoirs, the main challenge is the development of methods for volatilizing thermally labile molecules without breaking them apart. This will be critical for the detection of biomolecules, especially in combination with laser ionization for mass spectrometry.

\subsubsection{Classical Atomic Spectroscopic} Methods

With existing atomization techniques, attention must be given to two main areas: (a) the probing volume and temporal duty factor of the laser source in the atomizer and (b) the residence or transit time of the atoms in the probe volume. To achieve high sensitivity and the minimum sample size, atomic sources must be designed to match the shape and extent of the probe volume of the laser. Residence time depends upon the type of atomizer and the specific conditions present, such as gas flow velocity and atomizer probe volume. There are several types of classical atomic sources that have been used with laser-based methods and for which further development would be increasingly useful for trace analyses. Combustion flames and plasmas are widely used for atomic analyses with classical light sources and are known for their reliability, speed of analysis, multielement determination capability, and detection limits. These limits may lie in the $\mathrm{pg} / \mathrm{ml}$ range for some elements when laser-based methods are used (Skoog 1985; Christian and $0^{\prime}$ Reilly 1986). One of the most widely used of the plasma sources is the inductively coupled plasma (ICP) which, because of its high plasma temperature (7000-10,000 K) provides excellent atomization and thus remarkably high sensitivity $(\mathrm{pg} / \mathrm{ml})$ even for the most refractory elements. Classical atomic reservoirs include electrothermal atomizers such as graphite furnaces, boats, crucibles, and metal filaments. Graphite furnaces provide an extended probe time for enhanced sensitivity over flame methods. In addition, pyrolytic graphite furnaces, with an argon buffer, provide rapid atomization in a uniform temperature enclosure with almost negligible matrix interference. This lack of matrix interference is due to a temperature profile in which the background organic material is ashed out before the measurement is performed. Graphite furnaces combined with laser methods (fluorescence and ionization) have reached sensitivities in the attogram range.

\subsubsection{Glow Discharges}

A more recent development in atomic reservoirs is the glow discharge source. In this method, a discharge is formed in a pressure range of $<10$ torr. Cathodic sputtering occurs in the discharge with the result that its negative glow region becomes a reservoir of neutral sputtered atoms plus excited atoms and ions arising from the sputtered flux. In the absence of thermal evaporation, bulk diffusion, or surface diffusion, the composition of the material sputtered from a multielement solid under steady-state conditions reflects the composition of the bulk solid. The work of Harrison and co-workers (Harrison et al. 1986) has shown this source to be a useful method for trace analysis of materials on surfaces in combination with specific laser detection of the sputtered neutrals. This method has outstanding potential for trace analysis of metals and dielectrics which is of considerable interest to the semiconductor industry. Several interesting developments include the pulsed glow discharge which can be used effectively with pulsed laser sources. The second important development is the use of magnetically confined plasmas (McCaig et al. 1989) which can operate at relatively low pressure (10 $10^{-4}$ torr) and at sputtering currents equivalent to or greater than that of the glow discharge described 
above. These devices, called magnetrons, have great potential for rapid sputtering in the semiconductor industry.

\subsubsection{Laser-Induced Evaporation}

A very promising method for vaporizing both atomic solid samples and large biomolecules uses laser-induced evaporation (Lubman 1988a, 1988b; Grotemeyer and Schlag 1988; Hayes 1987; Coates and Wilkins 1987; Beavis et al. 1988). In the case of atoms, the process requires focussing a high-powered pulsed laser to ablate the atoms from the solid, creating a plume that may consist of neutrals and ions. In laser methods of atomic analyses, this type of atomization has several advantages: (a) an improvement in the duty cycle since a pulsed laser is used for vaporization and for subsequent laser excitation, (b) an improvement in the background since bulk heating of the sample is minimal, (c) achievement of high spatial resolution for microprobe experiments, (d) probing of both metals and dielectrics with minimal sample preparation, and (e) absolute sensitivity in the femtogram range.

In the case of molecules, a pulsed laser is used to desorb rapidly a sample from a surface before the molecules have time to kinetically decompose (Lubman 1988b). This is a much more gentle process than is atomic ablation since the sample is simply vaporized off the substrate surface and no apparent damage to the surface occurs. This process is generally thought to depend upon a rapid temperature increase on the surface in which ions and neutrals are produced in a ratio that depends on the temperature reached on the surface. In much of the work, a pulsed $\mathrm{CO}_{2}$ laser produces ions from a surface that are directly detected in a mass spectrometer. This laser has been used to detect compounds such as oligonucleotides and peptides in a mass spectrometer. More recently, UV sources have been used for direct desorption and ionization of bovine albumin at $M / z$ of $66,750 \mathrm{amu}$, other high molecular weight compounds (>10,000 amu) have been detected (Karas and Hillenkamp 1988).
In the desorption process, neutral molecules, rather than ions, generally form the bulk of the desorbed material. Several groups have thus used a second UV laser to induce ionization with the distinct advantages of (a) increased sensitivity due to the efficient multiphoton ionization (MPI) process, (b) enhanced selectivity based upon the spectral absorption features of the molecule in the MPI process, and (c) controlled fragmentation for structural analysis using MPI. Although this method has only been applied to molecules $<1,500 \mathrm{amu}$, it has the potential to have significant impact the detection of large biomolecules. There is considerable potential here for the development of this technique for application with classical enzymatic methods, to the sequencing of proteins and DNA fragments (see Section 5).

\subsubsection{Particle-Induced Vaporization}

Ion sputtering has been shown to be a very promising atomization method with the capability for high spatial resolution, thus making it suitable for the local analyses of solids and thin films with sensitivities reaching sub-ppb levels. The neutral atom yield, as a rule, is 2 to 4 orders of magnitude higher than the ion yield and is only weakly dependent on the matrix. The sputtering yield reaches its maximum when the ion energy is typically in the range of 5 to $50 \mathrm{keV}$. When the sputtering is employed to initiate the resonance ionization process, it is referred to as sputter-initiated resonance ionization spectroscopy (SIRIS) (Parks et al. 1983; for additional detail, see Hurst and Payne 1988). This technique has been used for sensitive depth profiling with important applications in the semiconductor industry. Winograd and his colleagues (Baxter et a1. 1986) have made important contributions to the understanding of ion sputtering and associated surface interactions using RIS.

In the molecular domain, Beavis et a1. (1988) have used fast atom bombardment (FAB), where a beam of 
neutral argon atoms is used as a means to induce desorption of small peptides from a substrate for detection by MPI. Ion desorption and electron-stimulated desorption (ESD) have also been used (by the Becker group at the Stanford Research Institute) to vaporize polymers and biomolecules for detection by MPI and VUV ionization (Schuhle et al. 1988) ESD has been found to be a very effective means of vaporizing fragile molecules with minimum decomposition. These techniques generally provide a much lower flux of material per unit time than does laser desorption, and thus it is necessary to use a high repetition rate for the detection laser in order to obtain reasonable sensitivity. Nevertheless, these methods will provide exciting opportunities for vaporizing large biomolecules and polymers for detection with mass spectrometers (see Section 5).

3.5.6. Supercritical and Liquid Injection in Jets

Liquid injection and supercritical fluid (SF) injection have served as a means of introducing nonvolatile molecules and chelated metals into mass spectrometers for some time (see, for example, Pang and Lubman 1988). The analyte of interest is dissolved in a solvent and injected as a gaseous jet into vacuum. Supercritical fluids present important opportunities since they can dissolve large molecules like a liquid but then expand as a gaseous jet in a vacuum. This presents excellent opportunities for selective detection by MPI or laser-induced fluorescence (LIF) based upon supersonic jet spectroscopy. The combination of SF injection and jet spectroscopy is being studied by several groups. The groups of Lee and Goates at Brigham Young University are developing methodology for $\mathrm{CO}_{2}$ and $\mathrm{N}_{2} \mathrm{O}$ capillary separation of polynuclear aromatic hydrocarbons (PNAH) in coal liquids with selective laser (LIF) spectroscopic detection. In recent work, Lubman and coworkers have used highly polar SF ammonia solvent to solubilize polar biological molecules such as nucleosides and small peptides. There will be important opportunities here for further investigation of polar SF solvents for extending this promising method to biological analyses. Other opportunities for volatilizing biological materials will arise from the use of highly polar liquid solvents for jet introduction. These methods appear particularly promising for interfacing with liquid separation techniques for the study of biological molecules in real matrices (see Section 5).

\subsubsection{Other Methods}

Several special techniques may be applicable where extreme trace detection is required. These include the atom buncher or multistage atomization method where the analyte is preselected by a laser-assisted separation technique and/or mass separator and is accumulated in a foil (ion implantation) before final atomization (by ion sputtering or laser ablation) takes place. These methods may also be applied to more stable molecular systems. Finally, the development of ion traps and laser cooling methods, where atomic or molecular ions or even neutral atoms can be held for long periods of time, would allow spectroscopic detection studies to be performed with impressive levels of accuracy and resolution.

\subsection{SYSTEMS}

\subsubsection{Laser Mass Spectroscopy Systems}

Mass spectrometry has proved to be a powerful tool for chemical analysis based upon exact mass/charge (M/ $q$ ) identification of molecules. The mating of lasers to mass spectrometers (MS) is a natural combination since direct laser desorption and MPI methods are often able to produce molecular ions or quasimolecular ions for identification; these methods have been extended for structural analysis of polymers, peptides, and ol igonucleotides (Lubman 1989; Grotemeyer and Schlag 1988; Campana 1987). In addition, these methods have been used to study PNAH's in coal fluids 
(Dobson et al. 1986) and coal macerals. The main limitation in this methodology is the relatively low repetition rate of most pulsed UV laser systems. In order to use scanning mass spectrometers with laser-induced MPI, continuous jet expansions with high repetition rate lasers $\left(>10^{4} \mathrm{~Hz}\right)$ must be developed. However, the alternative has been to use pulsed injection sources to produce high density pulsed molecular beams coupled with pulsed lasers. This requires the use of mass spectrometers such as timeof-flight (TOF) and fourier-transform mass spectrometry (FTMS) devices which can detect the whole mass spectrum on every laser pulse.

Early TOF devices were basically flight tubes with a diode source acceleration region. These early devices were capable of a resolution of several hundred at best. This may be the MS technology of the future for biological applications and analyses, especially in conjunction with laser desorption, laser microprobe, and laser MPI ionization sources. The most promising TOF-MS technology appears to be the reflectron. In this device, an ion reflector is used to reverse the flight direction of the ion packet. In the process, the initial energy spread of the ion packet is minimized and the resolution increases dramatically. Since its introduction by Mamyrin and co-workers (1973), modifications have increased mass resolving power to 10,000 (Grotemeyer and Schlag 1988; Campana 1988). Recently, one group has introduced a multipass reflection device (Sakurai et a1. 1985). In principle, these devices could provide ultrahigh resolution $(>100,000)$. Supersonic jet expansions used in conjunction with reflectron devices, provide enhanced resolution by further minimizing the energy spread in the initial sample of molecules.

Other methods having enhanced TOF resolution include the use of energy selectors or electric sectors in tandem with the TOF device (Campana 1988). Significant enhancement of resolution has been obtained using this method, especially in conjunction with gas chromatography (GC) and high performance Tiquid chromatography (HPLC) injection techniques. The most recent developments in resolution improvement for TOF have been achieved using velocity compaction. There are real opportunities here for developing sophisticated scanning electric fields for achieving very high resolution as shown by Enke's group at Michigan State (Yefchak et a1. 1989).

A second methodology that has been used with laser ionization and desorption is ion trapping, using ion cyclotron resonance devices (ICR) (Gross and Rempel 1984) and other forms of ion traps (Allison and Stepnowski 1987). The ICRMS utilizes a high magnetic field to cause ions to undergo a cyclotron motion in a small ion storage cell under vacuum ( $10^{-8}$ torr). According to the cyclotron equation, ions are mass sorted according to their frequency of revolution.

Very high resolution (in excess of $\left.10^{6}\right)$ has been obtained in the modern FTMS version of this device (Shomo et al. 1985), and thus it has tremendous capabilities for large biomolecule analysis. This method can be used in a tandem mode to elucidate MPI fragmentation and photodissociation mechanisms and for structural analysis of biomolecules. MPI or laser desorption may initially create several fragment ions, and the resulting fragments can be studied in the tandem mode to understand fragmentation patterns or the structure of a specific fragment of a biomolecule such as a DNA or protein. DOE is presently funding some work using this technique for the study of inorganic gas phase reactions mechanisms in the lab of Ben Freiser at Purdue. Some work on structural analyses using FTMS has been performed by several groups (see, for example, Lubman 1989).

The drawbacks of this method, relative to TOF devices, are the expense of the FTMS $(>\$ 300,000)$ and the lowpressure requirement. The latter has been partially solved using dual cell devices. Simple ion trap devices using combinations of $\mathrm{rf}$ and dc fields are now being used to trap ions in a storage cell at low pressures for hours, in some cases. A number of excellent reviews exist on the available modes of operations of these different traps (Gross and Rempel 1984). They can be 
used in a tandem mode very similar to FTMS. The mass resolution of these devices is not very high; however, they are relatively simple and inexpensive to build. They present excellent opportunities for detection of relatively small (<500 amu) labile biomolecules in conjunction with direct laser desorption and MPI methods.

\subsubsection{Laser Chromatographic Systems}

The development of microcolumn liquid chromatography during the present decade has led to significant improvements and new opportunities in HPLC measurements. Among the advantages provided by the microcolumns are higher efficiencies, improved detection performance, benefits derived from reduced flow rates, and the ability to work with smaller samples (Novotny 1988; Barth et al. 1988). Reduced flow rates have increased the mass sensitivity of concentration sensitive detectors. The microcolumns have also made it possible to exploit certain laser technologies for improving the detection sensitivity (Yeung 1986). These laser properties include high average-power, collimated beams, monochromatic beams, and time resolution (with pulsed beams). Raman scattering and fluorescence can be separated by using the time resolution feature.

Refractive index detectors can be improved significantly with the use of lasers. The reflection type of refractive index detector makes use of the light reflected at the glass/liquid interface when the incident beam is near the critical angle. The amount of light transmitted and reflected depends strongly on the refractive index of the liquid relative to the glass at the interface. As different components elute from the column, the liquid refractive index changes, causing a change in the amount of reflected light. The collimation of the laser beam increases this sensitivity. The size of the interaction region can al so be reduced by focusing the laser beam. Since only the region within a few wavelengths of the optical interface takes part in the interaction, the reflection type of refractive index detector can be used with very small volumes. The deflection type of refractive index detector can also benefit from the collimation provided by a laser beam, as can interferometric detectors. A Fabry-Perot interferometer has been used with a helium-neon laser to achieve a limit of detection (LOD) of $20 \mathrm{ng}$.

Absorption detectors are fundamentally limited by shot noise. The high power available from lasers makes it possible to approach saturation of the absorption transition. Intracavity absorption schemes have been used to obtain a LOD of $5 \times 10^{-5}$ absorbance units (Yeung 1986). Thermal lens calorimetry has been used with laser beams for HPLC detection. The intensity across a single-mode laser beam follows a Gaussian intensity distribution therefore more heating will occur in the center of the beam than at the edges. Observation of changes in the refractive-index gradient resulting from this heating has been used to obtain a $L O D$ of $1 \times 10^{-8}$ absorbance units in an $8 \mu \mathrm{L}$ flow cell. Absorbance measurements in liquid chromatography would be significantly improved by the development of compact, tunable UV lasers.

Laser-induced fluorescence as an HPLC detector is very attractive from the standpoint of sensitivity and versatility (Novotny 1988). High excitation intensity favors laser illumination. However, the major limitations are stray light, fluorescence or Raman scattering from the solvent, and fluorescence from cell walls and windows. The collimation of the laser beam helps to reduce the stray light problem. The monochromatic light from a laser helps discriminate between fluorescence and Raman light. A sheath-flow cuvette can be used to move the cuvette walls away from the column effluent. With this geometry, a LOD of $53 \mathrm{pg}$ has been reported for $8 \mathrm{~mW}$ of excitation at $488 \mathrm{~nm}$. (Herschberger et al. 1979). A flow cell using fiber optics in a fluorescence detection system has been developed and used to obtain a LOD of $1 \mathrm{pg}$ (Yeung 1986). A laser-based detector for HPLC has been developed that enables simultaneous absorbance, fluorescence, and refractive index 
detection for microcolumn liquid chromatography (Wilson and Yeung 1985). The refract ive-index detector can measure $6 \mathrm{ng}$ of benzene, the absorbance detector can measure $48 \mathrm{pg}$ of bromocresol green, and the fluorescence detector can measure $0.8 \mathrm{pg}$ of propylamine-NBD derivative. An argon laser at $488 \mathrm{~nm}$ is the light source. Another detection system uses a helium-cadmium laser at $325 \mathrm{~nm}$ and can measure the fluorescence from femtogram amounts of the dye coumarin 440 (McGuffin and Zare 1985). Fluorescence detection would be greatly improved with the development of compact, tunable lasers with wavelengths ranging from the UV to the IR.

Optical activity has been used as a chromatography detector (Yeung 1986). When an optically active eluent is used, all of the eluted components are detected unless they have the same optical activity as the eluent. If the same sample is first eluted with an optically active eluent and then eluted with a racemic mixture of the eluent, the eluted material can be quantified without the need for standards and without prior identification of the components.

Electrophoretic separations have been revolutionized by the development of capillary zone electrophoresis (Virtanen 1974; Mikkers et al. 1979; Jorgenson 1981,1983 ). In their review of capillary electrophoresis (Gordon et al. 1989), the authors state that the use of capiliaries as the electrophoretic migration channel will put CZE on the same footing as HPLC. In electrophoresis, molecules are separated on the basis of their electrophoretic mobility. The molecules to be separated are loaded into a tube filled with an electrolyte buffer, and a potential difference is applied across the ends of the tube. The capillaries in CZE have thin walls and inside diameters of $5.75 \mu \mathrm{m}$. The high surface-to-volume ratio means that heat is easily dissipated through the walls and convection does not occur. Resolutions equivalent to 1 million theoretical plates have been obtained (Lauer and McManigill 1986). Because of the small quantities of material that can be analyzed by CZE, the development of appropriate detectors is the major challenge.

Detection schemes for CZE include absorption, fluorescence, conductivity, electrochemical, mass spectroscopic, and radioisotopic methods. Direct absorbance in the UV has a detection limit of $10^{-6} \mathrm{M}$. Yu and Dovichi (1989) have recently reduced this limit by two orders of magnitude, using photothermal absorbance with a $130 \mathrm{~mW}$ argon-ion laser. Conductivity detection has a detection limit of $10^{-8} \mathrm{M}$ and has been used in CZE to detect metal ions, organic acids, and inorganic ions (Foret et al. 1986). Electrochemical detection limits with CZE have been pushed down to 200 to 400 attomoles for catecholamines in a $26 \mu \mathrm{m}$ inside-diameter capillary tube (Wallingford and Ewing 1987, 1988). An electrospray-ionization interface has been developed between a CZE apparatus and a mass spectrometer (Mack et al. 1970; Whitehouse et a7. 1985; 01 ivares et al. 1987; Smith et al. 1988). A CZE separation of ${ }^{32} \mathrm{p}-1$ labeled guanosine triphosphate and adenosine triphosphate injected at $5 \times 10^{-8} \mathrm{M}$ has been detected with an on-line radioisotope detector (Pentoney et a1. 1989). Cheng and Dovichi (1988) have coupled a flow cytometer to a CZE apparatus and used a fluorescence detector to push the limits of detection for fluorescing derivatives of amino acids to $5 \times 10^{-12} \mathrm{M}$ of alanine.

\section{REFERENCES}

Allison, J. and R. M. Stepnowski (1987) Anal. Chem. 59, 1072A-1086A.

Barth, Howard G., William E. Baker, Charles H. Lochmuller, Ronald E. Majors, and F. E. Regnier (1988) Anal. Chem. 60, $387 R-435 R$.

Baxter, J. P., J. Singh, G. A. Schick, P. H. Kobrin, and N. Winograd (1986) Nucl. Instrum. Methods in Phys. Res. B8, 345. Beavis, R. C., J. Grotemeyer, and E. W. Schlag (1988) paper presented at the 1988 
ASMS Conference on Mass Spectrometry and Allied Topics, San Francisco, CA.

Bowers, John E. and Charles A. Burrus (1987) J. Lightwave Tech. T-5, 1339-1350.

Brau, C. A., RECENT DEVELOPMENTS IN FREEELECTRON LASERS, Laser FocUs/ElectroOptics, Feb. 1987.

Bruce, D. M., R. J. Seymour, D. Cheong, P. E. Jessop, and B. K. Garside (1987) Can. J. Phys. 65, 924-928.

Campana, J. E., Ed. (1987) ANALYTICAL INSTRUMENTATION, Vol. 16 (Special issue on Time-of-Flight Mass Spectrometry Marcel Dekker, Inc.).

Campana, J. E., Ed. (1988) ANALYTICAL INSTRUMENTATION, Vol 17 (Special issue on Lasers and Mass Spectrometry - Marcel Dekker, Inc., New York).

Cheng, Y. F. and N. J. Dovichi (1988) Science 242, 562 .

Christian, G. D. and J. E. O'Reilly (1986), INSTRUMENTAL ANALYSIS (Allyn and Bacon, Boston), 2nd edition.

Coates, M. L. and C. L. Wilkins (1987) Anal. Chem. 59, 197-200.

Curran, S. C., A. L. Cockroft, and J. Angus, (1949) Philos. Mag. 40, 929.

Dobson, R. L. M., A. P. D'Silva, S. J. Weeks, and V. A. Fassel (1986) Anal. Chem. 58, 2129-2137.

Foret, F., M. Deml, V. Kahle, and P. Bocek (1986) Electrophoresis I, 430.

Gordon, M. J., X. Huiang, S. I. Pentoney, Jr., and R. N. Zare (1989) Science 242, 224-228.

Gross, M. L. and D. L. Rempel (1984) Science 226, 261.

Grotemeyer, J. and E. W. Schlag (1988) Angewandte Chemie 27, 447-592.
Harrison, W. W., K. R. Hess, R. K. Marcus, and F. L. King (1986) Anal. Chem. 58, 341A-356A.

Hayes, J. M. (1987) Chem. Rev. 87, 745-760.

Herschberger, L. W., J. B. Callis, and G. D. Christian (1979) Anal. Chem. 51, 1444.

Hurst, G. S. and M. G. Payne (1988) PRINCIPLES AND APPLICATIONS OF RESONANCE IONIZATION SPECTROSCOPY (Adam Hilger, Bristol, UK), Chapter 8.

Hurst, G. S., M. G. Payne, M. H. Nayfeh, J. P. Judish, and E. B. Wagner (1975) Phys. Rev. Lett. 35, 82.

Jorgenson, J. W. and K. K. Lukacs (1981) Anal Chem. 53, 1298.

Jorgenson, J. W. and K. D. Lukacs (1983) Science 222, 266.

Karas, M., and F. Hillenkamp (1988) Anal. Chem. 60, 2301-2303.

Kume, H., K. Koyama, K. Nakatsugawa, S. Suzuki, and David Fatlowitz (1988).

Lauer, H. H. and D. McManigill (1986) Anal. Chm. 58, 166.

Lubman, D. M. (1988a) Mass Spec. Reviews Z, 535-554.

Lubman, D. M. (1988b) Mass Spec. Reviews I, 559-592.

Lubman, D. M., Ed. (1989) LASERS AND MASS SPECTROMETRY (Oxford University Press, Oxford), in press.

Mack, L. L., P. Kralik, A. Rhonde, and M. Dole (1970) J. Chem. Phys. 52, 4977.

Mamyrin, B. A., V. I. Karataev, D. V. Shmikk, and V. A. Zagul in (1973) Sov. Phys.-JETP (Eng7. Trans1.) 37, 45-48.

McCaig, L., R. Sacks, and D. M. Lubman (1989) "Radiative and Electrical Properties of a Planar Magnetron Glow 
Discharge Device," Applied Spectroscopy (in press).

McGuffin, V. L. and R. N. Zare (1985) Appl. Spectroscopy $\underline{39}$, 847-853.

Mikkers, F. E. P., F. M. Everaerts, and Th. P. E. M. Verheggen (1979) J. Chromatogr. 169, 11.

Novotny, Milos (1988) Anal. Chem. 60, 501A-510A.

Olivares, J. A., N. T. Nguyen, C. R. Yonker, and R. D. Smith (1987) Anal. Chem. 59, 1230 .

0lesik, John W. and John P. Walters (1984) Appl. Spectros. 38, 578-585.

Pang, H. M., and D. M. Lubman (1988) Rev. Sci. Instrum. 59, 2460-2463.

Parks, J. E., H. W. Schmitt, G. S. Hurst, and W. M. Fairbank, Jr. (1983) in PROCEEDINGS SPIE CONFERENCE ON LASERBASED ULTRA-SENSITIVE SPECTROSCOPY AND DETECTION V, 426, Richard A. Keller, Chairman/Editor (SPIE - The International Society for Optical Engineering, Bellingham, WA), pp. 32-39.

Pentoney, S. L., Jr., J. F. Quint, and R. N. Zare (1989) Anal. Chem. 61, 1642.

Sakurai, T., Y. Fujita, T. Matsuo, H. Matsuda, and I. Katakuse (1985) Int. J. Mass Spectrom. Ion Processes 66, 283-290.

Schuhle, U., J. B. Pallix, and C. H. Becker (1988) J. Vac. Sci. Technol. A $\underline{6}(3), 936-940$.
Shomo, R. E., A. G. Marshall, and C. R. Weisenberger (1985) Anal. Chem. 57, 29402944.

Skoog, D. A., PRINCIPLES OF INSTRUMENTAL ANALYSIS (1985) (Saunders College Publishing, Philadelphia), 3rd edition.

Smith, R. D., J. 01 ivares, N. T. Nguyen, and H. R. Udseth (1988) Anal. Chem. 60, 436.

Virtanen, R. (1974) Acta Polytech. Scan. Chem. Incl. Metall. Ser. 123, 1-67.

Wallingford, R. A. and A. G. Ewing (1987) Anal. Chem. $\underline{59}, 1762$.

Wallingford, R. A. and A. G. Ewing (1988) Anal. Chem. 60, 258.

Whitehouse, C. M., R. N. Dreyer, M. Yamashita, and J. B. Fenn (1985) Anal. Chem. 57, 675 .

Wilson, Steven A. and Edward S. Yeung (1985) Anal. Chem. 57, 2611-2614.

Wojtczuk, S. J., J. M. Ballantyne, S. Wanuga, and Y. K. Chen (1987) J. Lightwave Tech. LT-5, 1365-1370.

Yeung, Edward S. (1986) in ANALYTICAL APPLICATIONS OF LASERS, edited by Edward H. Piepmeier (John Wiley, New York), pp. 557-586.

Yefchak, G. E., G. C. Enke, and J. F. Holl and (1989) Int. J. Mass Spectrom. \& Ion Processes (in press).

Yu, M. and N. Dovichi (1989) Mikrochim. Acta (in press). 


\section{STATUS OF LASER SPECTROSCOPY}

\subsection{INTRODUCTION}

"Spectroscopy" is the study of the energy levels of atoms, molecules and solids, as revealed in the characteristic optical lines which are absorbed or emitted in transitions between these levels. Before the invention of the laser in 1958, these spectral lines were studied with one of the two methods of classical spectroscopy -- (a) "emission spectroscopy" where the light emitted from an electrically or thermochemically excited sample is spectrally analyzed to reveal the characteristic emission lines, and (b) "absorption spectroscopy" where a continuum light source is passed through an absorbing sample and then spectrally analyzed to show the reduced intensity at the characteristic wavelengths. With the invention of the laser, light became available in a new form - - a highly directional, spectrally narrow and very intense beam -- and this has led to the development of many new methods of spectroscopy which have greatly increased our ability to study and to utilize atomic and molecular spectra. Many of the applications of these new techniques, such as sensitive detection of chemical pollutants and radioisotopes, are directly relevant to the OHER mission. The rapid pace of development of new laser sources and new methods of spectroscopy continues today, and judging from past experience, it is quite likely that many techniques which will be widely used in ten years time have not yet been invented. With this in mind, this section gives a brief review of the current status of laser spectroscopic methods, with an emphasis upon recent developments and trends which suggest future directions.

Almost all methods of laser spectroscopy involve a narrow-band tunable laser which interacts with a sample of atoms or molecules and which may, if its frequency matches one of the characteristic atomic frequencies, cause excitation of the sample. There are a variety of ways in which the presence of this excitation may be detected, however, and each defines a method of spectroscopy. Generally, while each method has advantages in some circumstances, none is preferable in all circumstances. In "absorption spectroscopy" the presence of sample excitation is detected through the consequent loss in intensity of the laser beam. In "fluorescence spectroscopy," the excitation is detected by collecting the photons which are spontaneously emitted as the excited sample decays. In RIS and its many variants, the laserinduced excitation has the effect of ionizing the sample atom, thus allowing for very efficient detection of the excited species in a mass spectrometer. "Photoacoustic spectroscopy" is a technique where a modulated laser beam generates periodic absorption of laser energy which is converted by collisions to a sound wave at the modulation frequency. "Photothermal spectroscopy" is somewhat similar except that the collisionally dissipated excitation energy is detected by the temperature rise which it causes in the sample. This can be sensitively detected by the thermally induced change in the sample's index of refraction. There are many variations on each of these schemes, and recent developments affect all of them. These are discussed briefly below.

In addition to the many new methods which have been developed to detect laser-induced atomic transitions, the high intensity of laser light has made it possible to induce atomic transitions which were unknown to classical spectroscopists, and which typically involve simultaneous absorption or emission of many photons. New light sources have spawned a whole area of investigation known as "nonl inear optical spectroscopy." Many of the new techniques developed in these investigations, such as "multiphoton 
absorption" and "stimulated Raman scattering," have become important spectroscopic tools in their own right and are also discussed below.

\subsection{FLUORESCENCE}

\subsubsection{Introduction}

Lasers have had the most profound impact on the techniques of atomic and molecular fluorescence, not only by pushing the detection sensitivities down to impressive levels -- close to single atom and molecule -- but al so by allowing the exploitation of entirely new experimental approaches in which photon irradiance, narrow spectral bandwidth, and temporal resolution combine to provide a unique tool suitable for chemical analysis as well as for fundamental spectroscopy studies. As a result, the entire field of basic and applied fluorescence has been revolutionized by the advent of lasers.

In combustion diagnostics, laserinduced fluorescence provides the attractive possibility of achieving simultaneously spatial and temporal resolution, addressing fundamental physical parameters such as temperature and number densities in the plasmas investigated (Lucht 1988). In chemical analysis, one reaches both sensitivity and selectivity which are essential in the field of microtrace analysis, i.e., the analysis of samples of limited sizes and of in situ microdistribution analysis. For example, it might be desired to study the distribution of an element in the various protein fractions rather than simply determine its natural ubiquitous concentration in the blood serum. The determination of an element at a concentration of $1 \mathrm{ng} / \mathrm{g}$ in an individual cell (ca. $1 \mu \mathrm{g}$ ) would then need a method with a detection power of $1 \mathrm{fg}$ (Tölg 1988).

An overview of the status and current trends in atomic and molecular fluorescence with laser excitation is reported below.

\subsubsection{Atomic Fluorescence}

Atomic and ionic fluorescence with laser excitation is a very versatile technique (Omenetto 1989). Several excitation/detection schemes are available by which the large majority of elements, including nonmetals, can be investigated. To accomplish this goal, the technique needs (a) one or two lasers, tunable from the low UV to the visible, with power sufficient to optically saturate the excitation transition chosen; (b) low background, efficient atomizers characterized by high values of quantum efficiency, i.e., low quenching; and (c) a highly efficient optical collection system since the fluorescence is isotropically emitted over $4 \pi$ steradians. The most favorable analytical scheme usualiy involves the use of nonresonance fluorescence transitions, i.e., different excitation and emission wavelengths, so that any nonselective scattering originating in the atom reservoir and occurring at the laser excitation frequency is avoided.

Detection limits for aqueous solutions nebulized in flames and plasmas are in the low nanogram-high picogram per milliliter range. Although this sensitivity is adequate for dedicated biological/bioclinical applications (e.g., the determination of some toxic elements like lead and cadmium in blood), in many instances higher absolute detection powers are necessary -- down to the femtogram level. The use of the technique has, therefore, been monopolized by atomizers which would allow reaching such powers of detection. Thermal vaporization with furnaces at atmospheric pressure or under vacuum has practically superceded flames and plasmas in the analysis of biological samples at ultratrace levels. For a few elements (e.g., silver, lead, thallium, and cadmium), absolute detection limits are in the low femtogram range and concentration detection limits are well below $1 \mathrm{pg} / \mathrm{ml}$ (assuming a sampling volume of a few tens of microliters). Indeed, for a given experimental setup, including pulsed laser excitation and atomization in a furnace tube surrounded by an argon atmosphere, the experimentally obtained 
detection limits are close to those which can be calculated theoretically, i.e., the technique is close to the ultimate detection sensitivities obtainable.

Two major trends can currentiy be identified. First with furnace atomizers, new methodologies are constantly being developed, aimed at avoiding furnace background emission and scattering. For example, the use of twostep excited fluorescence with detection in the low VUV has been successfully tested. Also, background correction techniques based on the Zeeman effect, the polarization of scattering, and nonlinear intermodulated fluorescence have been reported. Secondly, many elements, e.g., the refractory metals, are still not detectable at ultratrace levels. Therefore, increasing attention is devoted to atomizers such as pulsed glow discharges or to atomization methods such as laser ablation and ion sputtering. These atom reservoirs are coupled with the nonresonance, timeresolved fluorescence technique, thus avoiding scattering problems.

\subsubsection{Gas Phase Molecular Fluorescence}

The state of the art in gas phase fluorescence measurements is LIF in supersonic jets (SSJ) (Zare and Dadigian 1974, Hayes 1987, Smalley et a1. 1977 , Miller 1984, Levy 1980, 1984). The LIF method involves using a high-powered pulsed laser to selectively excite a molecule from the ground electronic state $\left(S_{0}\right)$ to an excited electronic state $\left(S_{1}\right)$. The total integrated fluorescence is then monitored as a function of wavelength. Although high sensitivity is obtained by monitoring the total fluorescence, selectivity is based upon the absorption of the narrow band laser radiation. The end result is that an excitation spectrum is obtained. Further, in the LIF method, dispersed emission obtained line by line in excitation provides a state distribution of the ground state molecules obtained from, for example, a reaction. Since most polyatomic molecules have broad unresolvable spectral features at room temperature, the SSJ method has been used to provide ultracold molecules with sharp spectral features for analysis (Hayes 1987, Lubman, 1987, 1988a, 1988b). This involves seeding a small percentage of the large polyatomic molecules in a large bath of a light carrier such as argon or helium and expanding through a pinhole orifice into vacuum. In the expansion, the energy of the internal molecular modes is relaxed through two-body collisions with the carrier gas, resulting in ultracold molecules. Since a pulsed laser is used for excitation, the SSJ is also generally pulsed to reduce vacuum pumping requirements. These methods have revolutionized detection and measurement in the gas phase, allowing for incredibly high sensitivity while obtaining selectivity and wavelength resolution.

To enhance fluorescence detection selectivity, a number of additional techniques have been interfaced to these measurements. These include dispersed fluorescence where an emission spectrum of the fluorescence is obtained. This increases the selectivity but lowers the sensitivity. Another method often used involves time-resolved measurements (Hayes 1987) for enhanced molecular identification.

Several trends can be noted in LIFSSJ. Thus far, most of the systems studied were PNAH's which have already been well characterized (Imasaka 1988). However, more recent work has extended this method to understand the basic spectroscopy of other classes of molecules such as $\mathrm{N}$ - and S-heterocycles, quinones, linear hydrocarbons and alcohols, and carboxylic acids. Several groups are interfacing chromatographic methods to supersonic jets for detection and identification of molecules in real matrices. This method appears particularly promising for identifying and quantifying components in complicated matrices such as PNAH's in coal (Hayes and Small 1982) or even small metabolites in blood and urine (Lubman 1988b). A number of methods have been developed for interfacing GC to LIF, including (a) direct capillary chromatography injection, (b) a pulsed injection method, and (c) a sheath flow method where the effluent of the GC is entrained within a 
fast flow of argon. In the case of nonvolatile compounds, limited work using supercritical fluids, liquids, or pulsed laser desorption has been used to entrain labile molecules into jets for LIF detection. However, much more extensive work, including instrumentation development, needs to be performed to utilize the capabilities inherent for detection of small biomolecules using these techniques.

\subsubsection{Condensed Phase Fluorescence}

The state of the art for obtaining selectivity in condensed phase fluorescence measurements appears to be matrix isolation spectroscopy at $4 \mathrm{~K}$ combined with fluorescence 1 ine narrowing (FLN) spectrometry (Wehry 1983, 1984; Perry et a1. 1983; Personov 1983; Sanders et al. 1986; Cooper et a1. 1988). The matrix may involve dissolving the material directly in liquid helium (or neon) or in amorphous glasses. The cold matrix is used to simplify the spectra of large molecules by cooling out the internal modes of vibration and rotation. However, solute molecules in the matrix still exhibit inhomogeneously broadened vibronic absorption bandwidths since the molecules locate a large number of sites. Thus, if a narrow band laser is used, it will select only a narrow subset of the solute molecules that absorb the laser radiation (Personov 1983). The selected set of molecules will fluoresce, and relatively narrow lines characteristic of the molecules are obtained.

This technique has been quite effective as a selective tool for identifying PNAH's which have relatively high fluorescent quantum yields and relatively sharp spectral features using FLN in matrices. Recently, work at Ames Laboratory has been performed on DNA-PNAH adducts using $F L N$ in amorphous glass matrices. Several adducts in laboratory mixtures were directly identified using the selectivity capabilities of this method. Using an intensified diodearray, optical multi-channel analyzer, the Ames group was able to detect an adduct of benzo[a]pyrene for a damage level of -5 bases in $10^{\circ}$. In more recent work, this group has used this technique to detect a PNAH-nucleoside adduct.

A second method widely used to obtain selectivity of fluorescent molecules in the condensed phase is synchronous fluorescence spectroscopy in which the excitation and emission frequencies $(\Delta \lambda)$ are scanned together to maintain a constant wavelength difference between them (Vo-Dinh 1978, Vo-Dinh and Martinez 1981). In this method, a signal is observed only when $\Delta \lambda$ matches the interval between one absorption band and one emission band. If one selects the $\Delta \lambda$ correctly to match one unique pair of absorption and emission bands, the synchronous spectrum will show only a single peak in an otherwise complicated spectrum. The resulting selectivity has been used to separate mixtures of PNAH's $^{\prime}$ using fluorescence spectroscopy, which otherwise could not be separated by this method. The excellent sensitivity of this method has been used to allow the measurement of a single adduct in at least $10^{7}$ DNA bases (Rahn et a1. 1982).

One of the present trends in solidstate fluorescence work appears to involve investigating microstructures in solid materials. Light microprobe techniques are diffraction limited so that new methods will be needed to study microstructures below $200 \mathrm{~nm}$. One new method being developed to overcome this limit utilizes exciton fluorescence and migration in solids. Exciton migration and fluorescence in the solid state has been a very active field for many years now. Recent work has shown that a pipette filled with anthracene can transfer energy to a target fluoroprobe in a spot of $<100 \mathrm{~nm}$. This method apparently uses UV laser light to excite one end of an anthracene crystal in which excitons are caused to migrate to the other end of the crystal in the tip of a pipette $<100 \mathrm{~nm}$ in diameter. The energy is confined to a very narrow region of space and is transferred to a fluorescing molecule at the pipette tip. If energy can be transferred in a region of space $<10 \mathrm{~nm}$, then this may be significant for detecting small structures in biological studies and even pinpointing the position of large fluorescence adducts in PNAH's 
in situ without excessive sample preparation.

\subsection{ABSORPTION SPECTROSCOPY}

While absorption spectroscopy is certainly not the most sensitive method of Taser spectroscopy, it has other advantages which make it preferable in many applications. For one thing, it is extremely general in its applicability. The use of absorption spectroscopy is not limited, for instance, to optical transitions whose upper state decays primarily (and rapidly) by fluorescence, but is equally effective regardless of the decay path of the excited levels. Secondly, it is a relatively simple method, in which the signal information is conveniently concentrated in a collimated laser beam. Finally, it is a nonintrusive method which is conveniently applicable to remote sensing applications. For all these reasons, absorption spectroscopy continues to be widely used. Actually, the fundamental sensitivity limits with absorption spectroscopy are much better than those usually achieved in practice. Shot noise in the laser intensity will determine the minimum detectable absorption, even in an otherwise ideal experiment, since these fluctuations will be mistaken for a real absorption signal. This, however, corresponds to very small absorptions. For instance, a $10 \mathrm{~mW}$ visible laser contains about $10^{16}$ photons/second; so with a one-second averaging time, absorptions as small as $10^{-8}$ should be observable within the shot noise limit. In practice, much larger absorptions are often difficult to observe, leaving considerable room for improvement in experimental methods of absorption spectroscopy.

One important recent development in absorption spectroscopy is the invention of techniques which circumvent the sensitivity limit due to the spectrum of amplitude noise of the laser source. Especially at low frequencies ( $<10 \mathrm{MHz}$ ) these intensity fluctuations can be many orders of magnitude greater than the shot-noise limit (Gehrtz et al. 1985). The basic idea of what has been called
"FM spectroscopy" is that by frequency modulating (FM) the laser source, the frequency derivative of the sample absorption is converted into an amplitude modulation of the transmitted laser intensity at the FM frequency. Since the FM frequency can be chosen to lie well above the frequency where 7 aser amplitude fluctuations are important, this allows the observation of shot-noise-limited absorption signals. This basic idea has now been well established by experiments using a wide variety of laser sources, and has made it possible to clearly observe absorptions as small as $10^{-7}$, consistent with the shot-noise limit. These relatively recent experimental advances in absorption spectroscopy should stimulate a reexamination of the relative merits of absorption spectroscopy in many applications.

A variation on simple absorption spectroscopy is "intracavity absorption spectroscopy, " where the absorbing sample is placed within the optical cavity of the laser oscillator. Since the output power of the laser is extremely sensitive to small losses in the cavity, this method is very sensitive to weak absorptions. A further variation is "laser magnetic resonance" where the intracavity sample is placed in a strong magnetic field. Through the Zeeman effect, tuning the magnetic field tunes the atomic transitions through resonance with the laser, without changing the laser frequency. This technique has found wide application in regions of the spectrum, such as the far-infrared (FIR), where tunable laser sources are unavailable or inconvenient but where many fixed-frequency lasers are known. Since the FIR is a rich spectral region, especially for molecules, laser magnetic resonance has been widely applied since its invention and has been used to study and characterize the spectrum of many molecules and molecular ions (Evenson 1981).

\subsection{RAMAN SPECTROSCOPY}

Spontaneous Raman spectroscopy, a weak, two-photon, inelastic scattering phenomenon, represents a general 
molecular effect that is applicable to any physical state and characteristic of all materials. Although electronic, vibrational, and rotational transitions can be probed by Raman spectroscopic techniques, laser-excited Raman spectra are most commonly used to provide information on the ground-state vibrational modes of systems spanning the size between diatomic molecules to macromolecular assemblies (Levin 1984). Raman spectra, arising from the intrinsic molecular polarizability changes that occur during nuclear motion, often complement the vibrational information obtained from IR absorption spectroscopy. IR data, however, are generated by a different molecular process in which the observed vibrational transitions are determined by gradients of the molecular dipole moment treated as functions of the molecular normal coordinates.

The Raman effect is described in terms of the molecular vibrational levels, as shown in Fig. 4-1. That is, laser photons, whose frequencies $\nu_{0}$ are generally in the visible region (for example, $514.5 \mathrm{~nm}$ or $19,436 \mathrm{~cm}^{-1}$ ) and nonresonant with any electronic level, excite the sample and create a "virtual" state from which photons are spontaneously (and inelastically) scattered at frequencies $\nu_{0} \pm \nu_{1}$. Ramanshifted radiation at $\nu_{0}-\nu_{1}$, which is recorded in "wavenumbers" $\left(\mathrm{cm}^{-1}\right)$, is termed Stokes-scattering and is the event that is commonly measured; radiation at $\nu_{0}+\nu_{1}$ represents the weaker, blueshifted anti-Stokes scattering. The numerical values of the Raman shifts correspond to the molecular vibrational modes found in the IR region of the spectrum from about 4000 to $10 \mathrm{~cm}^{-1}$. Thus, we recognize that the interaction of the laser radiation with the molecule is manifest by an increase in the natural vibrations of the system and that the Stokes scattered radiation simply specifies the normal modes of the vibrating molecule. Figure 4-1 also depicts both the anti-Stokes transitions and the Rayleigh scattering process in which the scattered light undergoes no change in wavelength.

The observed Raman intensity is proportional to the fourth power of the frequency of the scattered light, the magnitude of the exciting radiation, and the quantum mechanical transition probability for the vibrational levels under observation. As the exciting radiation approaches that of an electronic transition of a given chromophore within a molecule (see Fig. 4-1), the intensities of the vibrational displacement coordinates, within the specified structural moiety, that are involved in displacing the ground state geometry to the excited state conformation are selectively enhanced (Moore 1974). This phenomenon describes the resonance Raman effect, where increases in scattering enhancement can readily approach a factor of $10^{4}$, allowing systems to be probed at $10^{-3}$ to $10^{-7} \mathrm{M}$ concentrations. Resonance Raman approaches using both visible and UV tunable dye laser sources have been exploited both in analytical chemistry, where decreasing detection limits become critical, and in macromolecular systems in which chromo-phoric groupings representing loci for biological functions are conveniently probed. Although the Raman effect was discovered in 1928, the versatility of the technique -- coupled with the advent of a variety of laser sources, CCD, and diode-array detectors -- has literally led to multiple renaissances in the application of the method to problems in both physics and chemistry, as well as in the multidisciplinary biophysical and biochemical areas. Even today, we are again witnessing an explosion in applications of the technique as near-IR Nd:YAG lasers are coupled with Michelson interferometers in order to obtain Raman spectra of highly fluorescing molecular species. (In general, if fluorescence is induced by visible laser excitation, it will swamp the weak Raman signal and thus preclude an observation of the vibrational spectrum.) Since most biological materials exhibit strong fluorescence signals in the UV and visible, Fourier-transform Raman spectroscopy using near-IR laser sources offers an enormous potential for investigating entirely new classes of molecules. In addition, the spectroscopist now has at his disposal a 


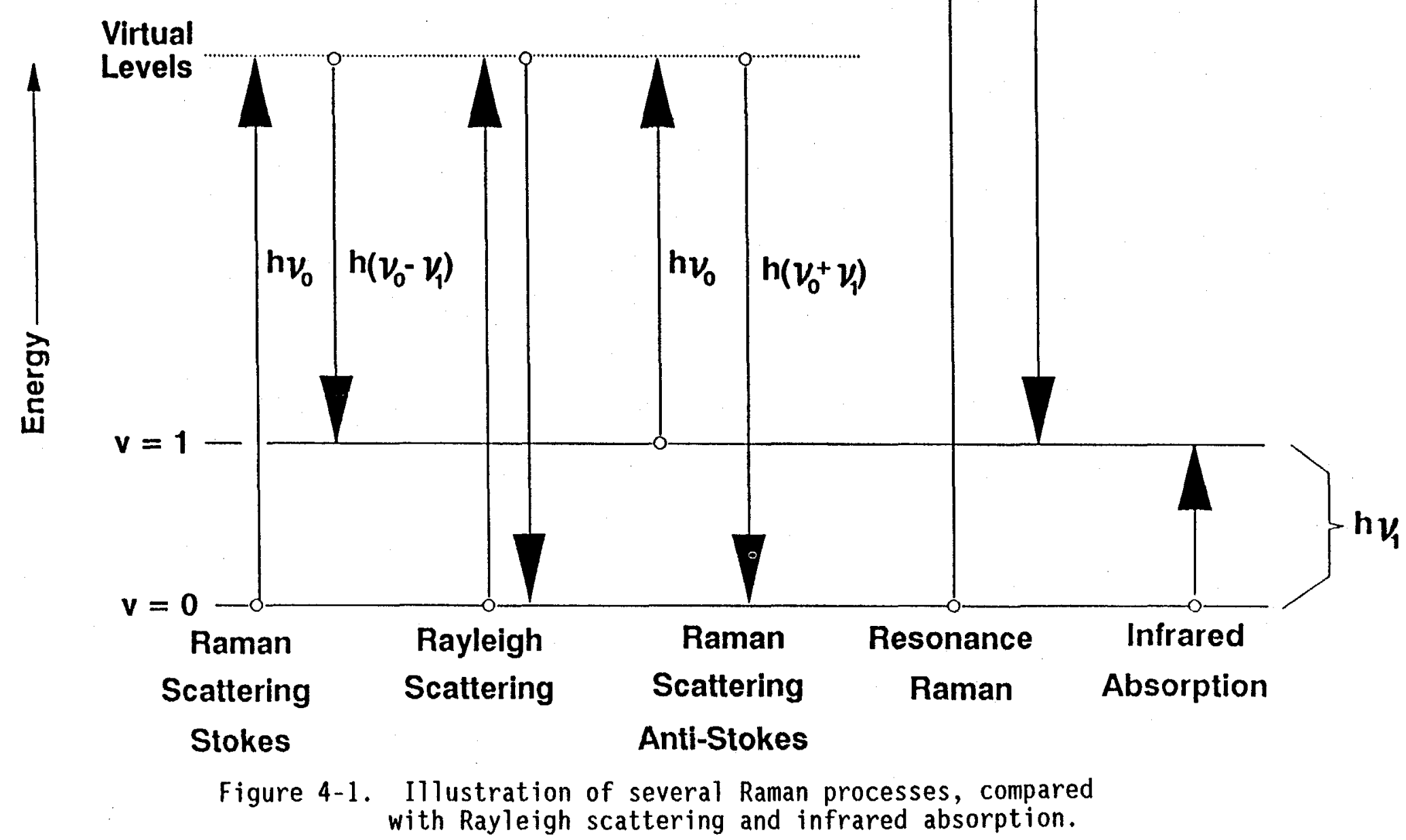


considerable number of new Raman spectroscopies, other than spontaneous and resonance Raman scattering, involving higher order Raman effects (Harvey 1981). These novel techniques have been used for a variety of diagnostic purposes and for observing spectral transitions not amenable to conventional Raman approaches.

\subsection{RESONANT AND MULTIPHOTON IONIZATION OF ATOMS}

Laser spectroscopy has dramatically improved the prospects for efficient ionization of atoms in the gas phase (Hurst and Payne 1988). Since these ions may be counted with essentially unit efficiency, a broad range of spectroscopic methods aimed toward exploiting this remarkable phenomenon are currently under development. These include basic spectroscopic studies of free atoms, detection of very small numbers of atoms in the gas phase, and, perhaps most importantly, development of methods to create gas phase atoms from solid or liquid targets which may then be subsequently counted by laser-induced ionization.

Figure 4-2 illustrates four elementary forms of laser ionization which are used for atoms and for molecules. For comparison, note that Fig. 4-2(a) illustrates the case of nonselective ionization with a single photon. In order to employ this process for the ionization of most atoms, it will be necessary to develop soft $X$-ray lasers with energies exceeding $10 \mathrm{eV}$. Process 4-2(b) is the most elementary example of RIS. In this case, it involves the excitation of a ground-state electron to a bound excited state, followed by the absorption of another photon to induce electron ejection or photoionization. More generally, the RIS process can include excitation via one or more discrete transitions, so that another photon of some convenient wavelength can complete the resonance ionization process. One remarkable feature of RIS is that the photoabsorption cross sections are generally quite large, allowing the use of defocused, low-power lasers. Also, selectivity factors approaching $10^{20}$ are possible since multiphoton absorption only occurs when the wavelength of the incident laser field is in proper resonance with the target atom. Using commercially available pulsed dye lasers, nearly 70 different elements have been ionized in the RIS mode (Parks and Lucatorto 1988). In many of these cases, the efficiency and selectivity have been shown to be near theoretical limits. There is also much excitement about recent successful attempts to resonantly ionize atoms with $\mathrm{Cw}$ lasers (Miller et al. 1982). Although the efficiencies are not as high as for pulsed lasers, with narrow bandwidths it has been possible to selectively ionize different isotopes of the same element (Miller et a1. 1985).

Another type of laser-based ionization process often used with atoms is illustrated in Fig. 4-2(d). This process involves the use of a high-power, tightly focused pulsed laser to unselectively pump enough energy into the target atom to cause photoionization (Becker and Gillen 1984). Usually, the MPI process of case (d) involves no resonance. However, in other cases, resonances may be accidental or deliberate to increase sensitivity and selectivity. Resonance-enhanced MPI, illustrated in (c), is an example of both RIS and MPI.

Multiphoton techniques provide new opportunities to learn about the basic behavior of atoms as they interact with strong laser fields. Line splittings due to the ac Stark effect, line broadening, and excited state lifetime measurements are leading to a more detailed understanding of nonlinear optical phenomena. For example, with this important information, it is now possible with near resonant transitions to routinely generate $115 \mathrm{~nm}$ radiation using "4-wave mixing."

In 1977, it was first demonstrated that a single atom of cesium could be observed in a background of $10^{19}$ argon atoms, using RIS and a proportional counter to detect the photoionized electron. The possibility of one-atom detection has created an avalanche of new 


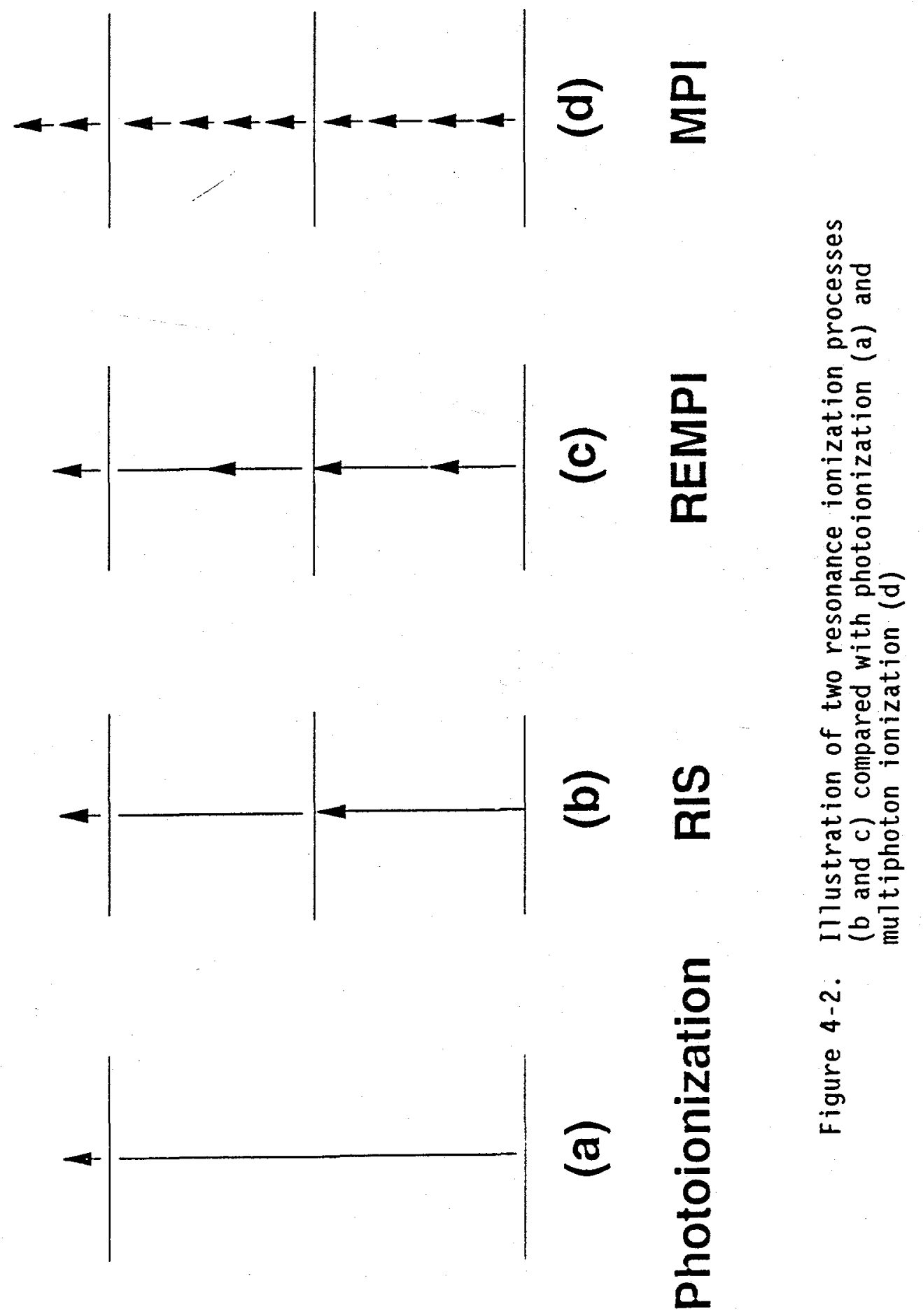


applications to exploit this unique experiment. It has been possible to observe the fluctuations of a few atoms in an inert gas and to examine a number of statistical mechanical concepts resulting from a small collection of atoms, including the ergodic hypothesis. Other applications include the detection of daughter atoms resulting from radioactive decay, the detection of short-lived radioactive isotopes such as ${ }^{221} \mathrm{Fr}$ with a half-life of $4.8 \mathrm{~min}$, and the determination of the solar neutrino flux. In this latter experiment, neutrino absorption by 862 tons of ethylene bromide is expected to yield 500 atoms of ${ }^{a 1} \mathrm{Kr}$. Because of its long half-life of $2.1 \times 10^{9}$ yrs, ${ }^{4} \mathrm{Kr}$ may be counted directly by RIS rather than by radioactive decay methods. The ${ }^{81} \mathrm{Kr}$ isotope has also been used in a number of geological applications.

The high selectivity and efficiency of RIS for atoms has spurred a great deal of research into controllable methods for atomizing solids. The earliest experiments involved simply heating a solid matrix in contact with a heated filament. The atomized sample is then ionized via RIS and detected with a mass spectrometer. The major problem with this scheme is that there is poor spatial and temporal overlap between the pulsed laser and the continuously evaporating source. Atomization using pulsed laser desorption (Hahn et al. 1988) or pulsed keV ion bombardment (Winograd et al. 1982; Parks et a1. 1983) has improved the efficiency tremendously. The latter method yields only atoms from the target surface, allowing the original gas-phase atom-counting experiments to be extended to surfaces (Pappas et al. 1989). These atomization techniques have also been coupled with nonresonant atomic ionization experiments. Measurements of this sort have proven to be important for the analysis of many chemical species simultaneously and are highly complementary to RIS (Becker and Gillen 1984). Al1 of these methods have not only been important new materials characterization techniques but have also allowed fundamental studies of the mechanism of laser ablation and ion/solid interactions.
An important application to analysis of solids is emerging via the use of $\mathrm{Cw}$ dye lasers. For atomized barium, it has been possible to achieve $6 \%$ ionization efficiency, using a doubly resonant, three-photon scheme where the ionization step was performed using a $1.7 \mathrm{~W} \mathrm{CO}_{2}$ laser (Bushaw et al. 1985). The narrow-band properties of $\mathrm{CW}$ lasers may be exploited to achieve a degree of isotope separation, using the hyperfine structure to distinguish each component. It has been possible to measure the isotope ratio of ${ }^{173} \mathrm{Lu}$ and ${ }^{174} \mathrm{Lu}$ on samples of less than $10^{-10}$ grams in which these isotopes are $10^{6}$ times less abundant than the more stable isotopes (Miller et al. 1985). The $\mathrm{CW}$ measurements are clearly exciting because of their enhanced selectivity and their improved "duty cycle" when compared to pulsed lasers. Developments in this area are particularly important to drive even more sensitive and demanding measurement applications. One-atom detection is of great importance to analytical chemistry. A related but inherently less sensitive method than RIS is the optogalvanic effect (OGE), which refers to the effect of light upon the electrical properties of a plasma. Though the earliest manifestations of the effect were observed in the 1920's and $1930^{\prime} \mathrm{s}$, it has been rendered far more general and useful by the advent of tunable lasers (Travis and Devoe 1981). The effect is functionally equivalent to collisionally ionized RIS, where the final ionization step is attributed to collisional effects in a buffer gas instead of photoionization. However, since ions are produced in the buffer gas, the attendant electrical background ensures that single atom events cannot be observed as they can with RIS in favorable environments.

The OGE in analytical flames is normally referred to as laser-enhanced ionization (LEI) in flames, and is sensed by impressing a high voltage across the flame and monitoring the increase in current synchronous with laser irradiation (Green 1986). For common 5 ns lasers, with powers capable of atomic optical saturation, a practical rule of thumb guarantees total ionization for population of an energy level within 
$1 \mathrm{eV}$ of the ionization limit. For the large portion of the periodic table with ionization potential (IP) values between 7 and $8 \mathrm{eV}$, this normally mandates a twostep excitation with a UV photon followed by a visible photon. Best-case detectabilities are about $1 \mathrm{pg} / \mathrm{mL}$ for metals in water aspirated into the flame, corresponding to number densities of around $10^{5}$ atoms $/ \mathrm{cm}^{3}$ in the flame. Detectability is degraded by sample components which ionize readily and increase the 1 imiting background current, or by nonresonant MPI of species in the flame. Unfortunately, LEI has not proved as adaptable to the atom reservoirs in current vogue in spectrochemical analys is as it is to the flame. The graphite tube furnace emits copious quantities of thermionic electrons (Sjostrom et al. 1988), and the inductively coupled plasma exhibits electrical incompatibilities and low sensitivities. Perhaps the most promising development for LEI since the flame is the thermionic-diode approach of Niemax et al. (1987). Sample vaporization is from a resistive filament, and ions produced by laserenhanced collisional ionization are detected with gains of several orders of magnitude due to space-charge amplification. The low-buffer gas pressure also permits high-resolution isotopic selectivity with Doppler-free cw laser spectroscopic techniques.

$$
\text { In a plasma, not in local }
$$
thermodynamic equilibrium (LTE), such as the low-pressure glow discharge, prediction of the sensitivity of the OGE is not as straightforward as in the LTE flame. Compared with the LTE model for flames, transitions between excited states and transitions involving lowenergy photons may be surprisingly sensitive in the glow discharge. The accessibility of high-lying transitions is attractive for $\mathrm{cw}$ laser excitation in the visible, and even IR diode-laser excitation in the near IR. Glow discharge OGE provides a convenient reservoir for atomic spectroscopy because natural sputtering of the discharge cathode by buffer gas ions may be used to provide free atoms directly from a solid electrode. The same feature might also be useful for solid sampling in chemical analyses.

Molecular and radical OGE signals have been obtained in both flames and discharges. Many radicals are most readily formed in discharges, and are thus particularly amenable to OGE spectroscopy. Similarly, many refractory metals form metal oxides in the analytical flame. Infrared, rovibrational transitions have also been detected optogalvanically in low-pressure discharges, including laser plasma tubes.

\subsection{MULTIPHOTON IONIZATION OF MOLECULES}

Note in reference to Fig. 4-2(a), it is possible to ionize many of the molecules with one photon. Photon energies exceeding $8 \mathrm{eV}$ or $155 \mathrm{~nm}$ are needed; therefore, the process must be performed in a vacuum. In practice, lasers are used for MPI of molecules in a manner similar to that in our discussion for atoms (Section 4.5) (Lubman 1988a, 1988b; Bernstein 1982; Gobeli et a7. 1985; Grotemeyer and Schlag 1988). In Fig. 4-2(d), where the photon is not in resonance with an electronic state in the molecule, the efficiency for ionization becomes small and high irradiance is needed to drive this process.

The truly unique property of resonance enhanced multiphoton ionization (REMPI) or RIS for molecules is the ability to obtain wavelength selectivity in the ionization process. Although ions are detected as the final product, the ionization cross section may reflect the absorption-excitation spectrum of the intermediate resonant electronic state of the molecule in the absence of other quenching mechanisms. In combination with the SSJ technique (see Section 4.2.2) which provides sharp spectral features, high optical selectivity can be achieved in the ionization process prior to mass spectral analysis. Thus, the possibility exists for highly selective optical-mass spectrometry which can be used to solve problems where sufficient selectivity cannot be easily achieved with conventional techniques. Such 
problems that are currently being investigated include trace detection of target compounds in a complicated matrix. This would have direct implications for detection of metabolites in blood and urine or for detection of trace toxic impurities in pharmaceutical syntheses where a discrimination of $1: 10^{\circ}$ may be required against a complicated matrix. Selective MPI (or RIS) has also proven valuable for detection of both isomeric and isobaric interferences which are difficult to distinguish by conventional methods. The most impressive demonstration of selectivity, however, has been in the area of isotopic detection, which is based upon the fairly sizable (several $\mathrm{cm}^{-1}$ ) vibrational shifts in the spectroscopy of isotopic molecules. Isotopic selectivity on the order of $1: 10^{8}$ has been demonstrated in the case of $\mathrm{I}_{2}$ isotopes using 1 aser REMPI in combination with mass selective techniques. The use of highly selective laser REMPI (or RIS) methodology may yet revolutionize current ideas held by the mass spectrometry community about how to perform mixture analyses.

A second important trend in this field is in the study of MPI-induced fragmentation (Bernste in 1982; Gobeli et a]. 1985). The processes for MPI-induced fragmentation are still not well understood, although studied by many groups. However, there may be an important opportunity here to use MPIinduced fragmentation of biomolecules (Lubman 1988b; Grotemeyer and Schlag 1988; $L i$ and Lubman 1988), such as peptides and nucleotides, for structural analyses and quality control in biotechnology or for detection of mutagenesis. MPI-induced fragmentation patterns are caused by absorption of photons by the molecular ion or by neutral fragments of the parent molecule. These fragment patterns are similar to those of electron impact (EI). MPI fragmentation in the near UV has been used to study aromatic-based peptides, and unique structural information can be obtained that is not easily available by alternate techniques. A real opportunity may afford itself here with the use of multicolor ionization-fragmentation schemes such as those demonstrated by Gobeli et al. (1985), where the fragmentation and spectroscopic states accessed can be selectively controlled for sequencing, based upon the precise energy of the photons used.

Another important trend is the use of direct VUV ionization of large biomolecules and polymers. Although direct VUV ionization has been used for some time with flashlamp sources, the development of VUV lasers which have high peak power and are tunable have already impacted this field. In recent work by Becker at the Stanford Research Institute (SRI) (Schuhle et a1. 1988), it has been shown that laser-generated VUV radiation at $118 \mathrm{~nm}$ can be used to obtain relatively soft ionization of large polymer units that have been desorbed using electron beam bombardment. Thus, relatively large polymers up to several thousand mass units can be studied in a mass spectrometer. VUV laser ionization may also allow direct examination of large biological molecules such as oligonucleotide strands for defects and, thus, may have significant impact on biotechnology quality control. The direct VUV method, however, is generally nonspecific and lacks selectivity (Arps et a1. 1989).

A new direction that is being employed is direct atmospheric pressure detection in situ, using MPI. Since near UV-1ight is transmitted through air, MPI can be performed in air at 1 atm and the ions detected by charge collectors or interfaced to MS. These atmospheric pressure MS methods have been shown to be capable of ppt detection and thus may be very important for detection of pollutants from coal burning plants and their subsequent transport through the air as one example.

Thus, the combination of tunable selectivity, high sensitivity due to the efficiency of ionization, controllable fragmentation for structural analysis, and the ability to interface MPI to mass analysis will make 1aser-MPI methods an invaluable tool for providing novel solutions to many problems in detection, separation, and structural analysis in various areas of chemistry and biology. 
4.7. PHOTOACOUSTICAL AND PHOTOTHERMAL SPECTROSCOPY

\subsubsection{Introduction}

These techniques represent outstanding evidence of the impact of lasers in absorption spectroscopy, allowing on one hand the attainment of unprecedented sensitivities and on the other hand the study of many physicochemical properties of the sample. Both techniques are based upon the generation of heat due to thermal de-excitation processes following an initial absorption step. The heat generated may be detected as a pressure change (optoacoustic or photoacoustic effect) or as a variation in the refractive index of the absorbing (adjacent) medium (photothermal effect).

To the extent that the matrix containing the analyte is nonabsorbing, we are dealing with zero-background measurement techniques, with capabilities of reaching absorption detection 1 imits as low as $10^{-8} \mathrm{~cm}^{-1}$, which could transiate into concentrational detection limits of $10^{-12}$ to $10^{-13} \mathrm{M}$, for strongly absorbing complexes. At these exceptionally low absorbencies, solvent absorption and its associated noise will limit the ultimate detection power available. In principle, chemical speciation is also possible and in some cases, e.g., actinide ions in water, at concentration levels which are inaccessible to other conventional, nonlaser techniques. Although not an intrinsic characteristic of the techniques, spectral selectivity can be improved by coupling these methods with gas, liquid, and thin-layer chromatographic methods.

\subsubsection{Photoacoustical Spectroscopy}

Several

applications of photoacoustics (PA) have been described (Tam 1986). PA imaging is concerned with the detection of subsurface thermoelastic property variations in a sample. If little lateral resolution is required, one speaks of PA depth-profiling, while PA microscopy is reserved for the case where lateral resolution is also achieved. PA depth-profiling may be uniquely suited for certain in vivo studies in medicine and biology (e.g., dermatology and cancer diagnostics), while PA microscopy has been used for nondestructive imaging of a variety of subsurface features (e.g., holes in metals, defects in integrated circuits, foreign inclusions in biological samples). PA techniques are becoming increasingly useful for the measurement of spectroscopic properties of biomolecules in vitro and in vivo (Moore et a1. 1983).

In fact, other techniques (e.g., transmission and reflectance) are not suitable for the study of biological materials due to opacities, scattering, heterogeneous surface properties, etc. The PA technique can be used to measure concentration gradients, e.g., the sedimentation of humic acids (Wylie and Lai 1986).

The sensitivity of the PA signal to the size of particles (within a given range) was exploited in the photoacoustic immunoassay method (Kitamori et al . 1987), anticipated as being applicable to determinations for trace tumors and cancer marker antigens, such as $\alpha$ fetoprotein, basic protein, and carcinoembryonic antigen.

of particular relevance is the possibility to couple PA measurements with fluorescence to study the status of green matter, and thus the efficiency of the photosynthetic system as a function of various types of stress (water and nutrient deficiency, herbicides, gaseous pollutants, toxic metals).

In the analytical spectroscopy field, PA methods have been applied to a variety of gaseous and liquid samples, with sensitivities at the parts per billion/parts per trillion levels. As an example, the speciation of actinides in underground water (Pollard 1985) is of utmost relevance for understanding the transport and migration of these species in the soil/water system (see also Section 5.2.3).

An extremely attractive way to enhance drasticaliy the photoacoustic effect is by initiating or terminating a chemical chain reaction (amplification or quenching effect) (Choi and Diebold 
1985). In this way, nanomole and picomole sensitivities have been demonstrated for several gases.

PA methods are well established. Discernible trends are found in the efforts made to further improve the sensitivity and to increase the number of applications, including spectral regions other than visible or near UV.

\subsubsection{Photothermal Spectroscopy}

In these methods, the heated sample acts as an optical element, leading to a rich lexicon of techniques. For example, a lens in thermal lensing, a grating in thermal diffraction, or a prism in thermal deflection (Dovichi 1987).

One or two beams can be used. Crossed beams provide excellent spatial resolution: for two beams crossing at right angles, the probed intersection volume may be as small as femtoliters if the laser spot size is of the order of micrometers, leading to sensitivities in the low femtomole/high attomole range (e.g., for amino acids). Such resolution and detection capabilities are of clear value in chromatographic, electrophoretic, and microscopic imaging and detection.

Supercritical fluid chromatography is another promising area, in which more than 100-fold sensitivity improvements have been reached. Surface analys is is possible by the so-called "mirage effect" (photothermal deflection of the probe beam) or by "reflection" measurement probing the surface deformation. Studies of thermal properties of materials have been reported.

As in the photoacoustic case, speciation of actinides and Ianthanides in solution is possible. Colloidal solutions can also be investigated. As a typical example, the absorption properties for dissolved organic carbon, which plays an important role in the speciation of trace metals, was studied in fresh waters (Power and Langford 1988).

Miscellaneous applications include the detection of ultratrace metals in liquids (e.g., phosphorous, cadmium, copper, iron, and cobalt), the analysis of gases at sub-ppb concentration levels, and the quantification of proteins separated by polyacrylamide gel electrophoresis.

The same expectations and conclusions made for photoacoustic techniques hold here. A clear trend in photothermal spectroscopy is to reduce the size and complexity of the experimental setup (e.g., compacting the apparatus and using single-beam configuration, in which pump and probe experiments are feasible).

\section{REFERENCES}

Arps, J. H., C. H. Chen, M. P. McCann, and I. Datskou (1989) "Ionization of Organic Molecules Using Coherent Vacuum Ultra- violet Light," Applied Spectroscopy (submitted).

Becker, C. H. and K. T. Gillen (1984) Ana1. Chem. 56, 11.

Bernstein, R. B. (1982) J. Phys. Chem. 86, 1178-1184.

Bushaw, B. A., T. J. Whitaker, B. D. Cannon, and R. A. Warner (1985) J. Opt. Soc. Am. B2, 1547 .

Choi, J. G. and G. J. Diebold (1985) Anal. Chem. 57, 2989.

Cooper, R. S., R. Jankowiak, J. M. Hayes, L. Pei-qi, and G. J. Small (1988) Anal. Chem. 60, 2692-2694.

Dovichi, N. J. (1987) CRC Crit. Rev. Anal. Chem. 17, 357.

Evenson, K. M. (1981) Discuss. Faraday Soc. $71,7$.

Gehrtz, M., G. C. Bjorklund, and Edward A. Whittaker (1985) J. Opt. Soc. Am. B 2, 1510. 
Gobeli, D. A., J. J. Yang, and M. A. ElSayed (1985) Chem. Rev. 85, 529-559.

Green, Robert B. (1986) in ANALYTICAL APPLICATIONS OF LASERS, edited by E. H. Piepmeier (John Wiley \& Sons, New York), pp. 75-105.

Grotemeyer, J., and E. W. Schlag (1988) Angewandte Chemie 27, 447-592.

Hager, J. W., and S. C. Wallace (1988) Anal. Chem. 60, 5-10.

Hahn, J. H., R. Zenobi, J. L. Bada, and R. N. Zare (1988) Science 239, 1523 .

Harvey, A. B., Editor (1981) CHEMICAL APPLICATIONS OF NONLINEAR RAMAN SPECTROSCOPY (Academic Press, New York).

Hayes, J. M. (1987) Chem. Rev. 87, $745-760$.

Hayes, J. M., and G. J. Small (1982) Anal. Chem. 54, 1202-1204.

Hurst, G. S. and M. G. Payne (1988) PRINCIPLES AND APPLICATIONS OF RESONANCE IONIZATION SPECTROSCOPY (Adam Hilger Ltd., Bristol, U.K. and Philadelphia).

Imasaka, T. (1988) Spectrochimica Acta 43B, 661-669.

Kitamori, T., K. Suzuki, T. Sawada, and Y. Gohshi (1987) Anal. Chem. 59, 2519.

Levin, I. W. (1984) in ADVANCES IN INFRARED AND RAMAN SPECTROSCOPY, Edited by R.J.H. Clark and R. E. Hester (Wiley Heyden, New York), Vol. 11, pp. 1-48.

Levy, D. H. (1980) Ann. Rev. Phys. Chem. 31, $197-225$.

Levy, D. H. (1984) Sci. Am. 250, 96-109.

Li, L., and D. M. Lubman (1988) Anal. Chem. 60, 1409-1415.

Lubman, D. M. (1987) Anal. Chem. 59, $31 \mathrm{~A}-40 \mathrm{~A}$.

Lubman, D. M. (1988a) Mass Spec. Reviews I, 535-554.
Lubman, D. M. (1988b) Mass Spec. Reviews I. 559-592.

Lucht, R. P. (1988) in LASER SPECTROSCOPY AND ITS APPLICATION, edited by Radziemski, R. W. Solarz, and J. A. Paisner (Marcel Dekker, Inc., New York), Chapter 9.

Miller, C. M., R. Engleman, and R. A. Keller (1985) J. Opt. Soc. Am. B2, 1503.

Miller, C. M., N. S. Nogar, A. J. Gancarz, and W. R. Shields (1982) Anal. Chem. 54, 2377.

Miller, T. A. (1984) Science 223, 545-553.

Moore, C. Bradley, Editor (1974) CHEMICAL AND BIOCHEMICAL APPLICATIONS OF LASERS (Academic Press, New York).

Moore, T. A., E. P. O'Hara, D. M. Aujo, R. Tom, and D. Benin (1983) J. Physique, Colloque C6, Suppl. No. 10,339.

Niemax, K., J. Lawrenz, and A. Obsrebski (1987) in RESONANCE IONIZATION SPECTROSCOPY 1986, edited by G. S. Hurst and $C$. G. Morgan (Institute of Physics, Bristol, U.K.), pp. 45-50.

Omenetto, N. (1989) Spectrochim. Acta $\underline{44 \mathrm{~B}}, 131$.

Pappas, D. L., D. M. Hrubowchak, M. H. Ervin, and N. Winograd (1989) Science, in press.

Parks, J. E. and T. B. Lucatorto, Eds. (1988) RESONANCE IONIZATION SPECTROSCOPY 1988, Conference Series No. 94 (Institute of Physics, Bristol, England).

Parks, J. E., H. W. Schmitt, G. S. Hurst, and W. M. Fairbank Jr. (1983) Thin Sol. Films 108, 69.

Perry, M. B., E. L. Wehry, and G. Mamantov (1983) Anal. Chem. 55, 1893-1896.

Personov, R. I. (1983) in SPECTROSCOPY AND EXCITATION OF CONDENSED MOLECULAR SYSTEMS, edited by V. M. Angranovich and 
R. M. Hochstrasser (North-Holland Publishing, New York, NY).

Pollard, P. M. (1985) Harwell Report AERE-R11496.

Power, J. F. and C. H. Langford (1988) Anal. Chem. 60, 842.

Rahn, R. 0., S. S. Chang, J. M. Holl and, and L. R. Shugart (1982) Biophys. Res. Commun. 109, 262-268.

Sanders, M. J., R. S. Cooper, R. Jankowiak, G. J. Small, V. Heisig, and A. M. Jeffrey (1986) Anal. Chem. 58, 816-820.

Schuhle, V., J. B. Pallix, and C. H. Becker (1988) J. Vac. Sci. Technol. A $6(3), 936-940$.

Sjostrom, S., J. Lejon, and H. Rubinsztein-Dunlop (1988) in RESONANCE IONIZATION SPECTROSCOPY 1988, edited by T.B. Lucatorto and J.E. Parks (Institute of Physics, Bristol, U.K.), pp. 151-154.

Smalley, R. E., L. Wharton, and D. H. Levy (1977) Acc. Chem. Res. 10, 139-145.
Tam, A. C. (1986) Rev. Mod. Phys. 58, 381.

Tölg, G. (1988) Fresenius Z. Anal. Chem. 329, 735 .

Travis, John C. and James R. DeVoe (1981) in LASERS IN CHEMICAL ANALYSIS, edited by Gary M. Hieftje, John C. Travis, and Fred E. Lytle (Humana Press, Clifton, NJ), pp. 93-124.

Vo-Dinh, T. (1978) Anal. Chem. 50, 396-401.

Vo-Dinh, T., and P. R. Martinez (1981) Anal. Chim. Acta 125, 13-19.

Wehry, E. L. (1983) Trends Anal. Chem. (Pers. Ed.) 2, 143-147.

Wehry, E. L. (1984) Anal. Chem. 56, 156R-173R.

Winograd, N., J. P. Baxter, and F. M. Kimock (1982) Chem. Phys. Lett. 88, 581.

Wylie, I. W. and E. P. Lai (1986) Rev. Sci. Instr. 57, 1185 .

Zare, R. N. and P. J. Dadigian, (1974) Science 185, 739-747. 


\section{POTENTIAL IMPACTS ON THE OHER PROGRAMS}

\subsection{INTRODUCTION}

In this section we discuss the impacts that the present status and especially the trends of laser technology (Section 3) and laser spectroscopy (Section 4) are having on programs with in the OHER mission. A number of suggestions are made which can be very useful to those engaged in laser-related research and development. Further, most of the prioritized recommendations made in this report are based on these suggestions. For convenience of identification, the suggestions are italicized.

\subsection{LASER-BASED METHODS FOR ELEMENTAL ANALYSES}

Samples which are normally solids, liquids, or molecular gases must be rendered into free atoms or atomic ions before identification of the atomic species is possible. Thus, among the earliest applications of the laser to atomic spectrochemical analyses was the optical laser microprobe, in which laser ablation was employed to sample a small portion of a sample for selective detection by optical emission spectroscopy (Laqua 1979). In spite of continuing research in the use of laser ablation for spectro-chemical analyses (Cremers and Radziemski 1987), the commercial optical laser microprobe has been displaced by the Laser Microprobe Mass Analyzer, commercially offered by Leybold-Heraeus as the LAMMA and by Cambridge Consultants as the LIMA (Houk 1986). In these instruments, ions generated directly in the laser-induced plasma are mass-analyzed by TOF mass spectrometry. Laser microprobes can be used to advantage for microanalytical applications to biological specimens, inclusions in alloys, ceramics, plastics, polymers, glasses, and to dust particles (Piepmeier 1986). They are considered to be primarily qualitative (or semiquantitative) instruments, at best, with reasonably high sensitivities, but subject to matrix effects and sensitivity variations among analyte species. A significant advantage of the laser microprobe over charged particle microprobes is the applicability to the study of nonconducting samples.

The selectivity provided by the interaction of tunable laser radiation with the characteristic energy levels of atomic species is the critical aspect of many of the most important applications of Tasers to chemical analysis. RIS, LEI, and LIF selectively detect atoms of the analyte species in the presence of large excesses of concomitant atoms.

From the discussion of atomic ionization methods in Section 4.5, it may be seen that ongoing research needs for analytical application are:

1) Studies of the basic physical mechanisms of the laser ablation process for its use in the laser microprobe. Laser ablation is widely researched for a variety of applications beyond chemical analyses, but a thorough understanding of the mechanisms for a wide variety of complex materials is missing. Many artifacts which hamper sensitive and accurate analyses may be related to fluctuations in energy per pulse, and in spatical and temporal mode instabilities.

2) Adaptation of new laser sources, especially continuous wave, to resonance ionization. The widespread analytical application of RIS and resonance ionization mass spectroscopy (RIMS), as well as the commercialization of the method, has been severely limited by the nature of available tunable lasers. With the approaching revolution in tunable lasers, as envisaged in Section 3.2, a new era for analytical laser spectrometry may be beginning. 
3) Development and characterization of excitation and ionization schemes for all elements amenable to spectrochemical analysis. Broadly tunable, all-solidstate systems with reasonably accurate and simple wavelength control would both raise the demand for proven, tabulated RIS schemes, and greatly assist in the exploration of potential schemes.

\section{4) Further development of techniques} for the vaporization of samples of arbitrary composition in vacuum and controlled atmospheres, yielding accurate representation of the bulk sample and efficient geometric and temporal overlap with pulsed lasers as well as $\mathrm{CW}$ lasers. To the extent that new laser sources can be continuous, instead of pulsed, the potential analytical impact is even greater. However, certain excitation schemes will probably cont inue to require the extended wavelength range and/or multiphoton capability of pulsed lasers. For these cases, suitably pulsed sampling schemes are essential to attaining the ultimate efficiency in sample use. Laser ablation/desorption and pulsed ion beam sputtering are among the prime candidates.

5) Development of isotopically selective (high resolution) ionization methods for hybridization with mass spectrometry and/or LIF for detection of low abundance, long-lived radioisotopes in the presence of large excesses of stable species. Again, isotopic selectivity may be aided in many cases by the use of new Cw laser systems, with implications for chemical analysis as well as isotope separation (Bushaw et al. 1987; Bushaw and Gerke 1988). A7so, Niemax et al. (1987) propose the use of optical isotope-dilution quantitation without the use of mass spectrometry.

6) Further implementation and characterization of alternate ionization methods (e.g., $d c$ and rf field ionization, collisional ionization) for RIS. Though fully capable of saturating resonant atomic transitions in vacuum, $\mathrm{CW}$ sources may need the assistance of highpowered IR lasers for efficient photoionization (see Section 4.5) or may benefit from alternate ionization methods. Pulsed-dc field ionization, as widely practiced in the Soviet Union, is of particular interest to reduce some of the 1 aser requirements.

7) Continued development of technology unique to RIS of rare isotopes of inert gases. The "Maxwell's demon" approach to isotopically selective raregas detection has been successfully adapted from the work at Oak Ridge National Laboratory (Hurst et al. 1985) by Atom Sciences (Lehmann et al. 1987; Thonnard et al. 1987), and is presently capable of quantifying a few hundred atoms of ${ }^{81} \mathrm{Kr}$ in the presence of $10^{11}$ atoms of ${ }^{82} \mathrm{Kr}$ and $10^{25}$ molecules of water. The process relies entirely on mass spectrometric isotopic selectivity, requiring several stages of isotopic enrichment prior to the final atom count using RIS. Future generation devices have been separately proposed by Payne (1988), Hurst et al. (1988), and Hardis et a1. (1988) to reduce the number of stages required for the process.

8) Develop position sensitive
ionization detectors.
Ionization
electors such as proportional counters,
dectron multipliers, and single-ion
setectors can be provided with "position
sensitivity". Therefore, laser
ionization processes can, in principle,
be used to construct spatial images
showing the locations of the atoms or
molecules that were ionized. This
capability has not been exploited in the
past, yet it illustrates another
interesting potential use of lasers in
the future.
Another major impact of lasers on
atomic analysis is in the area of LIF or
laser excited atomic fluorescence
spectrometry (LEAFS) as it is often known
when applied to analytical atom
reservoirs (see Section 4.2 .2 ).
Consideration of Section 4.2 .2 suggests
a number of important development areas


which should be supported to enhance the analytical future of LEAFS:

1) The development of new methodologies to avoid background emission and scattering with furnace atomizers. The use of two-step excited fluorescence, with detection at shorter wavelength than either excitation, should be extended to more elements. Zeemaneffect background correction techniques should be applied to a variety of real samples. Polarization-based rejection methods and nonlinear techniques such as intermodulated fluorescence, used widely in laser spectroscopy, need to be fully implemented in the analytical context.

2) UV sources for initial excitation. Many elements of critical importance, such as arsenic, selenium, tellurium, and phosphorus, have evaded analytical laser spectrometrists, because their principal resonance excitation lines at energies above $40,000 \mathrm{~cm}^{-1}$ were not conveniently accessible to lasers. The recently developed BBO crystal is solving this problem. This area is relevant to RIS as we 11 as LEAFS.

\section{3) Multiphoton fluorescence} investigations. With pulsed laser excitation, the photon irradiance is high enough for efficient excitation (often to "virtual" levels) of many nonmetals (oxygen, fluorine, chlorine, nitrogen, and carbon) which can then be detected by measuring their red fluorescence emission.

4) New atomizers. Many elements, such as the refractory metals, are still not detectable at ultratrace levels. Atomizers such as pulsed glow discharges or atomization methods such as laser ablation or ion sputtering should be tested with careful time-gated detection of nonresonant emission. Requirements for atomization and light collection should be met in one device, designed to provide the maximum efficiency for both.
5) Standardless analyses. In some cases, modern graphite furnace technology and atomic absorption can provide absolute (standardless) analyses in several complex matrices to with in $10 \%$ to $20 \%$ accuracy. The same technology could possibly be applied to optically saturated fluorescence measurements with calibrated detectors.

6) Continued development of the photon-burst method for single atom detection by LIF. Photon-burst methodology is being developed with DOE funding at Los Alamos National Laboratory and at Pacific Northwest Laboratory. Keller et al. at Los Alamos National Laboratory propose the use of isotopically selective photon burst detection on an ion beam which has already undergone magnetic mass discrimination, thus convoluting the selectivities of the two methods (Fairbank et al. 1988). Whitaker and Cannon (1988) at Pacific Northwest Laboratory are applying photon burst methods to rare gases excited to metastable states in discharges.

\subsection{ENVIRONMENTAL RESEARCH}

\subsubsection{Global Effects and Active Remote Sensing}

The fact that human activities are now altering the content of the global atmosphere justifies increased measurement. Fortunately, measurements can now be made that will allow us to investigate and learn about the global environment and perhaps eventually learn to model the global climate. The tools at hand are observation from aircraft and space craft platforms, using both active and passive monitoring approaches. NASA has recently completed a detailed study of measurements that should be performed from space platforms as part of the Earth observation system proposal. This study was used as one scientific component of the proposal placed before Congress this year called the Mission to Planet Earth. The program called for measurements on a 
global scale not only of atmospheric parameters such as wind, temperature, pressure, humidity, cloud height, carbon dioxide and other gases, and atmospheric turbidity but also for measurements of global sea conditions, including temperature, sea state, and sea height, and also for measurements of global geodesy with a precision not before possible. In other words, over the next decade the earth would be studied as a system from aircraft platforms or orbiting polar satellites and from the space station.

The Department of Energy may have the opportunity to cooperate with NASA in the Mission to Planet Earth program and support measurements of atmospheric parameters that are impacted by energy generation. The goal of the program would be to provide guidance to long-term energy production policy that is in the interest of the United States and the world community.

Active remote sensing includes microwave sounding, radar imaging of clouds and rain, and height measurements to the ground. A great number of parameters can be measured by active sensing in which a signal of electromagnetic radiation is transmitted, scattered or absorbed by the object, and detected in either transmission or in reflection. Opportunities for active laser remote sensing include the detection of clear air turbulence (CAT) from aircraft to enhance the safety of the vehicle and its passengers, the measurement of wind shear near airports, the measurement of atmospheric temperature, pressure, humidity, turbidity, tropopause height, global dust dispersion from either volcanic activity or other large events, and global wind measurements. All of the laser remote sensing approaches involve either inelastic scattering processes such as absorption and remission of optical radiation on or off resonance, or elastic scattering as a return from a solid object such as the ground or from particles in the atmosphere. In the former case, tunable laser radiation is required to selectively monitor the molecule or atom of interest. In the latter case, highly coherent radiation is desired, especially if wind velocity is to be determined from Doppler-shifted return signals.

To date, remote sensing using tunable laser sources has been limited to ground-based or to aircraft platforms. However, laser technology is now being developed by NASA that will allow early test measurements to be made from the shuttle bay. The laser technology is based on flashlamp-pumped Nd:YAG followed by harmonic generation to the green to pump a tunable solid-state laser or a tunable dye laser. It is expected that the laser source will transmit approximately $1 \mathrm{~J}$ of energy at a repetition rate approaching $10 \mathrm{~Hz}$. The receiver optics is a $1 M$ diameter reflector mirror. The first system will use direct detection of the received signal with time resolution providing the vertical spatial resolution for the measurement.

The wind sensing measurements are being approached using coherent detection to resolve the Doppler-shifted frequency. The first generation system is based on the $\mathrm{CO}_{2}$ laser at the $10 \mathrm{\mu m}$ wavelength band. Studies based on ground observations have shown that the $\mathrm{CO}_{2}$ laser system is of adequate sensitivity for wind sensing using currently available laser amplifier technology. However, there remains some question about the feasibility of packaging the $\mathrm{CO}_{2}$ laser for satellite-based global observations because of the operational lifetime of the laser, the high voltage required for the excitation of the gas, and the lack of return scattered signal at the $10 \mu \mathrm{m}$ wavelength from particles in the atmosphere. These issues have opened the possibility that a solid-state laser transmitter could be developed for global wind measurements.

The diode-pumped, Nd:YAG laser oscillator followed by a sixty dB gain laser amplifier was the basis for the first coherent laser radar measurement in the $1 \mu \mathrm{m}$ wavelength range. The first experiments of coherent laser radar used the coherence of the diode-pumped, nonplanar, Nd:YAG, ring-laser oscillator in a ground-based demonstration. With the rapid development of the diode-laser technology, diode-pumped Nd:YAG lasers 
now appear to be quite capable of making the transition to coherent laser radar in both aircraft and spacecraft platforms. However, the 1 um wavelength of the Nd:YAG transition is not eye-safe which places severe constraints on the ability to use the laser in an uncontrolled environment. Work began three years ago to define a diode-pumped laser system that would meet the eyesafe requirements. The work led to the demonstration of a diode-pumped Tm:Ho:YAG laser which operates at the $2.1 \mu \mathrm{m}$ wavelength region. Also Er:YAG lasers operating at $1.54 \mu \mathrm{m}$ are currently used for eyesafe applications. This wavelength is eyesafe and is transmitted through the atmosphere free of absorption. Further, work on the laser system showed the potential for efficient operation and for high peak power operation in a Q-switched mode. There is now considerable effort to develop both the efficiency and the coherence of the diode-laser-pumped Tm:Ho:YAG laser source for wind sensing or clear air turbulence applications. In addition, this laser source could also form the basis for coherent laser radar for near-distance measurements of both speed and target size and location.

In summary, the motivation for diodepumped, solid-state laser research conducted in the early 1980's was global remote sensing of atmospheric parameters including chemical constituents, density, temperature, humidity, aerosol concentration, and wind velocity. The requirements for the laser transmitter operating from an $800 \mathrm{~km}$ altitude orbiting satellite include a 4 year operational lifetime, greater than $10 \%$ electrical efficiency, $100 \mathrm{~W}$ of average output power in a linewidth of less than $10 \mathrm{kHz}$, and operation at an eyesafe wavelength. With the recent demonstration of a diode-laser-pumped Tm:Ho:YAG operating at room temperature at the eyesafe $2.1 \mu \mathrm{m}$ wavelength, a crucial step has been taken to achieve the above goals. It now appears feasible to define the all solid-state laser transmitter that will allow depthresolved global wind measurements by the mid 1990's.
The Mission to Earth program proposed by NASA for the next decade is now recognized as one of the most important space missions ever defined. By participating with MASA to develop the remote sensing technology for global remote sensing, DOE has the opportunity to play a major role in seeking answers to questions of global importance related to the effects of energy on the climate.

An attractive application of active remote sensing methods is the use of LIF of green plants in order to gain some insight about the functioning of their photosynthetic apparatus, i.e., the possibility to challenge LIF as an early detector of a potential status of stress. In fact, under optimum conditions of photosynthesis, the proportion of absorbed light which is emitted as red chlorophyll fluorescence is rather low, while under various conditions of stress (e.g., water deficiency, lack of nutrients, presence of herbicides, toxic metals, and gases) the fluorescence emission increases considerably. There is, therefore, an inverse relationship between in vivo chlorophyll fluorescence and photosynthetic activity (Lichtenthaler and Rinderle 1988; Chappelle and Williams 1986).

The incorporation of lasers into oceanographic technology is well documented in a NASA report ( $\mathrm{Kim}$ and Ryan 1975) where several hydrographic light detection and ranging (LIDAR) applications involving the fluorescence technique are reported, including the mapping of phytoplankton and oil spills. Airborne fluorescence emission signatures obtained from vegetation were also reported in the literature (Hoge et a1. 1983). Work at NASA/Goddard Space Flight Center (Chappelle and Williams 1986) involved the use of a nitrogen laser $(337 \mathrm{~nm}, 30 \mathrm{~Hz}$, and $9 \mathrm{~mJ} /$ pulse with a pulse width of $10 \mathrm{~ns}$ ) to excite, in a laboratory configuration, the fluorescence spectra of all major plant types (i.e., herbaceous, dicots, monocots, conifers, hardwoods, and algae) with the aim to correlate the fluorescence measurement with the manifestations of specific environmental 
changes. Fluorescence spectra not only provided a means for plant type identification but also reflected the status of physiological stresses.

Since LIF is amenable to aircraft and, potentially, to space platform observations, there is an opportunity to complement already existing standard remote sensing methodology, e.g., passive reflectance measurements, with the addition of a fluorosensor package (which can be operated with a fixed frequency UV laser as excitation source and an optical multichannel analyzer for the simultaneous detection of the spectral information.)

\subsubsection{Transport Studies Involving Actinides and Heavy Elements}

As noted in the previous section (5.3.1), modern laser spectroscopy and laser technology can make a significant impact on the important studies concerning the fate and transport of many elements and/or chemicals, both natural and synthetic, throughout the atmosphere/soil/water system. This is essentially due to the fact that lasers provide an analytical laboratory capability, as described in Section 5.2, with much improved characteristics of sensitivity and selectivity. As a result, the range over which measurements can be made is considerably extended.

Laser techniques can be used in remote sensing to trace directly gaseous pollutants (e.g., $\mathrm{SO}_{2}, \mathrm{NO}_{x}, \mathrm{O}_{3}$ ); these measurements help to elucidate the dynamical response patterns of the global tropospheric chemical system (National Academy Press 1984). Here, many additional opportunities exist to increase the selectivity. For example, the use of artificial intelligence to unscramble complex signals may be of particular value. Moreover, air samples can be collected at various sites and analyzed in the laboratory, producing data from which atmospheric transport patterns can be discerned.
Large quantities of transuranic elements are produced from commercial nuclear technology as burn-up products of uranium fuel. The disposal of long-lived radioactive waste has largely involved the need of finding deep, geologically stable sites (e.g., clay and granite) with long pathways to the biosphere and good absorptive properties for the actinides that might be leached out of the waste itself (Kim 1986; Saunders and Wilkins 1987). Any assessment of the consequences of a potential release of actinides from such repositories relies upon the ability to predict the migration behavior of these elements in soil/water systems, i.e., it requires the characterization of their absorption process by many potentially receptive organisms.

The key word common to these studies is "chemical speciation," i.e., in its broadest meaning, the capability to identify and quantify inorganic and organometallic compounds, as well as organic compounds, present in the environment (Braman 1983). Speciation studies should be stressed when preparing environmental impact statements, since, due to large differences in volatility and solubility, some chemical forms can dominate the physical exchanges occurring in global geochemical cycles so that the rates of decomposition and transportation of the various elements are strongly affected. Moreover, the biological activity (e.g., toxicity) of an element can vary significantly from one compound to another. Since the solubility of transuranic compounds in near neutral solutions is very small $\left(<10^{-8} \mathrm{~mol} / \mathrm{L}\right)$, their direct speciation under natural conditions seems to be feasible only by laser spectroscopic methods.

Thermal lensing spectrophotometry and photoacoustic spectroscopy (see Section 4.7) have been applied to the determination of uranium, plutonium, americium, and neptunium in natural waters under varying conditions of $\mathrm{pH}$, Eh, and carbonate concentrations with sensitivities attaining $10^{-8} \mathrm{~mol} / \mathrm{L}$, i.e., absorption coefficients of approximately $10^{-7} \mathrm{~cm}^{-1}$.

The combination of laser spectroscopy and chemical sensors seems 
to offer the most promising new method of in situ chemical analysis of the environment (see Section 5.3.4). The inherent miniature dimensions of these sensors have elicited considerable interest not only in the environmental field (e.g., air samples, potable waters) but also in the biomedical field (e.g., body fluids) where useful applications for direct "dip-in" operations have been found.

Remote monitoring of groundwater contaminants can be successfully tackled with fiber optic-based probes and fluorescence detection, especially when coupled with time resolution (Kenny and Jarvis 1987; Vodacek and Philpot 1987). In this case, naturally fluorescent contaminants can be detected directly; in situ, specific fluorescent-based optrodes can be developed; or, finally, a fluorescent tracer can be injected in the water and the motion of the fluorescent plume followed. In the case of uranium, the fluorescence lifetime persists for a comparatively long time (few tens of microseconds) as opposed to that of most organic molecules present in natural waters. Therefore, time gating of the signal allows rejection of the interfering species with very high detection sensitivities. Aromatic groundwater constituents (e.g., barkpile and landfill leachates, phenol, cresol, gasoline, and organochlorides) can be detected.

Particularly important are the complex organic acids found in natural waters and variously called humic substances, dissolved organic carbon, Gelbstoffe, or, more specifically, fulvic or humic acids. Because of their affinity with metal ions in general, they provide an important mechanism for the transport, dispersion, and sedimentation of metals in natural waters. The biological availability of these ions, both as nutrients and as toxins, is altered by complexation to humic substances.

RIS (see Section 4.5) has provided a new method for dating water which has applications to both marine and terrestrial environments. Very sensitive methods for counting individual atoms with isotopic selectivity have been employed in these applications. For instance, ${ }^{85} \mathrm{Kr}$ has been used to date polar ice caps to gain information on climatic history, and ${ }^{81} \mathrm{Kr}$ could be used in a very similar way for extensive analyses of groundwater. These methods could be used on a much wider scale for tracing the migration of pollutants which could affect our water supply. of particular importance is the use of ${ }^{81} \mathrm{Kr}$ to date water in wells up to 1 million years. The question of whether these wells have been recently vented to modern sources can be addressed by measuring ${ }^{85} \mathrm{Kr}$ with a half-life of 10.7 yrs.

\subsubsection{Fossil Fuel Pollutants}

As a result of either natural pyrolysis processes or fossil fuel burning, PNAH's are widely distributed in our environment. Their release into air and water can directly affect the health of individuals since the formation of adducts between DNA and PNAH's may be important in initiating carcinogenesis (Butler 1979; Reddy et al. 1984). Although the level of PNAH concentration can be monitored in the environment, a more useful indication of the risks of mutagenesis and carcinogenesis can be obtained by actually measuring the level of DNA-PNAH adducts found in the body. In particular, the ability to accurately identify the adduct can tell investigators something about the source of the contaminant, i.e., whether it came from a coal-burning plant, an oil spill, a forest fire, etc. In turn, the major source of mutagenesis in any one region may be pinpointed and controlled by such methodology.

A number of chemical methods have been developed to study the extent of PNAH-DNA adduct formation, including radiolabelling methods (Reddy 1984). However, the accurate identification of DNA-PNAH adducts requires high selectivity using ultrasensitive detection techniques. One laser-based method which has been used to achieve this goal is synchronous fluorescence spectroscopy (Vo-Dinh 1978). This method 
can provide enhanced selectivity based on relatively narrow linewidths for absorption and sensitivity based on fluorescence detection to allow the measurement of a single adduct in $10^{7}$ DNA bases. However, the measured linewidths are still too broad to distinguish between chromophores that have severely overlapped absorption bands. A more selective method is the use of fluorescence line narrowing spectrometry in combination with liquid helium or glass matrices at $4.2 \mathrm{~K}$. This method was used to distinguish DNA-PNAH adducts ind various mixtures with an estimated detection of 5 bases in $10^{6}$ (Sanders et a). 1986). The latter has also been combined with enzymatic degradation and TLC separation to further separate the nucleotides from the adduct-nucleotide pairs (Cooper et al. 1988).

The methods described above may be sufficiently selective for a limited number of DNA-PNAH adducts; however, if a large variety of adducts from different fossit fuel sources are present, then more selective techniques need to be developed. This may require actual DNA sequencing procedures (see Section 5.4). Because of the large number of base pairs in human DNA $\left(3 \times 10^{\circ}\right)$, rapid sequencing methods need to be developed which should be able to provide unambiguous identification and to distinguish against modified nucleosides, which is difficult by conventional methods. The ability to obtain unique identification and to distinguish modified nucleosides can be obtained using mass spectrometric methods. Future directions in this field will require development of efficient introduction and volatilization methods for these highly polar molecules following enzymatic degradation. New developments in continuous flow FAB are now having a major impact on peptide sequencing and identification and with modification should be applicable to nucleotide sequencing (Caprioli et al. 1986). other techniques to be investigated include various particle desorption methods which can be used to volatilize nucleotide strands. These can then be analyzed by a combination of mass spectrometry and fragmentation pattern recognition.
Recently published work (Nuwaysir and Wilkins 1989; Lam et al. 1988) has demonstrated the ability to volatilize, detect, and identify nuclei acid strands up to 10 base sequences long. In combination with enzymatic methods that are selective towards specified cleavages, this method may yet provide the additional speed needed to achieve rapid sequencing.

\subsubsection{Chemical Sensors (Optrodes)}

As already reported in Section 5.3.2, there is a significant need for accurate measurement of the concentrations of a variety of chemical species associated with energy production. Lasers and fiber optics led to the invention of the chemical optrode, the optical equivalent of a chemical sensing electrode. The optrode concept is simple. The tip of a fiber optic is coated with a chemical that undergoes some optically measurable change on exposure to another chemical in its immediate environment. This change is measured, for example, by transmitting a light beam down the fiber and sensing the amount of light at a specified wavelength that returns from the tip.

Optrodes have been developed to measure the concentrations of a wide variety of chemical species (Goldman 1988; Saari 1987; Wolfbeis 1986). Many optical parameters, such as absorbance reflectance, chemiluminescence, and Raman scatter have been used up to now; others, including 1 ight polarization, decay time, and refractive index can still be exploited. Metal ions such as beryllium, aluminum, magnesium, zinc, and cadmium can now be detected using fluorescencebased optrodes. Concentrations of oxygen, carbon dioxide, and hydrogen ion (pH) can also be measured. Optrodes have been developed to measure hydrogen sulfide, methanol, formaldehyde, phenols, and various chlorinated hydrocarbons.

It is now possible to measure the concentrations of chemicals at sites that were not feasible only a few years ago.

optrodes could be placed in water wells to continuously monitor the 
concentrations of a variety of pollutants. Optrodes could be buried at various depths surrounding a chemical waste disposal site to monitor the concentrations of the waste chemicals and track their movement in the soil. Some pollutants are transported in the soil by anaerobic bacteria. Movement of these pollutants might be monitored by using buried optrodes to measure waste products of bacteria that are transporting to the pollutant. Optrodes could be implanted in laboratory animals exposed to chemical pollutants and the concentrations of the pollutants and their metabolites monitored in vivo. This might significantly reduce the number of animals needed in such research projects.

Enzyme-based optrodes, which are a recent development, greatly increase the specificity of chemical measurements. As an example, an optrode has been developed for measurement of the concentration of p-nitrophenylphosphate (Arnold 1985). Alkaline phosphatase is immobilized on a nylon mesh at the tip of a fiber optic. The alkaline phosphatase catalyzes the hydrolysis of p-nitrophenylphosphate, producing $p-n i t r o p h e n o x i d e$, which absorbs light at $404 \mathrm{~nm}$. Light is transmitted down the fiber optic and the amount of light backscattered at $404 \mathrm{~nm}$ is monitored. When the enzymatic reaction occurs, some of this light is absorbed by the p-nitrophenoxide, enabling the measurement of the concentration of p-nitrophenylphosphate.

The concentration of penicillin can now be measured with an enzyme-based optrode (KuTp et al. 1987). Penicillinase and the fluorescent compound fluorescein are mixed in polyacrylamide gel covalently attached to the end of a fiber optic. When penicillin diffuses into the gel from the bulk solution, penicillinase catalyzes the cleavage of the B-lactam ring of penicillin, producing penicillic acid which dissociates into penicillate and a proton, producing a $\mathrm{pH}$ change in the sensor medium and a consequent decrease in the fluorescence of the fluorescein. This change in fluorescence is detected synchronously, using a chopped light source and a lock-in amplifier. The minimum detectable concentration is
$250 \mu \mathrm{M}$ of penicillin $\mathrm{G}$. There are opportunities for development of new sensor types to add more selectivity to the measurement (while still maintaining adequate sensitivity) and to increase the number of applications, especially since small, tunable lasers and high transmittance of fiber optic cables have made many new wavelengths accessible. In particular, the development of new enzyme-based optrodes should be encouraged. Many enzyme-based reactions are well understood. In addition, the enzyme attached to the end of the fiber is not consumed in the reaction, so the detectors should have a reasonably long lifetime.

\subsection{BIOLOGICAL RESEARCH}

\subsubsection{Imaging}

Biological and medical sciences have made the greatest advances when a new tool was developed to reveal biological structures in more detail. The first major advance was the invention of the microscope which revealed for the first time the world of microorganisms and the cellular nature of living organisms. The next leap was the discovery of $X$ rays which permitted a view of the internal structure of large organisms. This was followed by the electron microscope which permits a view of external structures on a much finer scale but only after the structures are immobilized and stained or coated with metal films. The organisms are no longer in their living form, but still great progress has been made with this very important capability. A more recent advance is the MRI technology which has similar or somewhat better resolution than does the medical $X$-ray technology, but it permits the visualization of the internal structure of hydrogen-containing or soft tissues and organs of the human body. The most recent advance is the scanning tunneling microscope which can determine surface profiles to subatomic dimensions.

$A$ new technology is now under development which should permit the next 
stride in biology and medicine -- that of $X$-ray holography. Holograms in the extreme ultraviolet (XUV) region in the water window with resolution of 3 to $5 \mathrm{~nm}$ could reveal internal structure of living matter the size of a cell. Later developments should permit holograms with .5 to $1 \mathrm{~nm}$ resolution (at the level of larger molecules). However, the difficulties increase very rapidly below $1 \mathrm{~nm}$, making the achievement of such resolution very speculative.

Even the achievement of resolutions of 1 to $10 \mathrm{~nm}$ requires the development of two separate technologies. The first is an X-ray source of the appropriate intensity and coherence length. The second is an imaging system based on holography.

The brightest synchrotron light sources now planned have enough intensity, even after selecting a narrow enough spectral width with a monochromator, to give the desired coherence length (about $1 \mu \mathrm{m}$ ) by using an expanded exposure time ( $-5 \mathrm{~min}$ ) for $1 \mathrm{~nm}$ resolution. This means, of course, that any sample must be completely immobilized for that time, and radiation damage will be limiting in many cases. $X$-ray lasers under active development, as discussed in Section 3.2.4, should be able to provide a source capable of producing a hologram of living material in a single exposure which is too short in duration to permit blurring (the order of $-10^{-13} \mathrm{~s}$ ).

Holographic systems are of two general types. The first is the Fresnel or the Gabor hologram which uses the original wave as a reference to interfere with the scattered wave from the target to form the hologram and so produces no magnification. This means that the recording medium or device must have the desired resolution. Photo resists have the best resolution $(-10 \mathrm{~nm})$, a major limitation. The second is the Fourier transform hologram. This uses a spherical reference wave so that the magnification can be chosen to match the recording medium. The resolution is set by the magnitude of the scattering angle accepted by the recording medium $(a=\lambda / 2 \theta)$. The required reference wave can be generated by focusing part of the primary beam through an appropriate pinhole or by the use of a metal scattering sphere of the appropriate size to give the desired reference wave intensity. Pinholes and zone plates for focusing limits resolution to $20 \mathrm{~nm}$. However, the resolution of $1 \mathrm{~nm}$ with a source wavelength of $.6 \mathrm{~nm}$ should be obtainable with the sphere technique. A system is under development by a group in Chicago which uses a reference sphere, an $X$-ray sensitive array CCD with a computer readout, and appropriate software to reconstruct the desired video images from the resulting hologram. Optical reconstruction techniques have also been developed.

If successful, this technique could be used on many other important problems in biology. The most important development can only be achieved if both the $X$-ray laser and the higher resolution holographic schemes are developed and used to obtain three dimensional structural information on biological molecules and living systems such as viruses and organelles. By selecting the wavelength, the resulting image would be atomic-species selective, greatly increasing the information obtained and improving the effective resolution. This technique would also freeze the action $\left(\sim 10^{-13} \mathrm{sec}\right)$ and thus reveal structure and conformational changes. These appear to be a key to the understanding of biological activity. Other groups are known to be exploring direct imaging techniques, but information on these activities is not generally available.

\subsubsection{Fast Chemical Kinetics}

The development of mode-locked lasers at wavelengths ranging from IR to the UV; fast, high sensitivity detectors; and picosecond streak cameras have made it possible to investigate chemical kinetics on the nanosecond to femtosecond time scales. These developments have the potential for making major contribution toward understanding some of the basic physics, chemistry, and biology programs of OHER. 
In general, the techniques are the time-dependent measurements of optical absorption by use of variable delay between an exciting beam and a time-locked probe beam to determine saturation and relaxation rates, or by photon-excited fluorescence which is then observed by streak cameras to resolve very short time scales. Channel plates and diode detectors are used for longer time scales, together with a sampling oscilloscope or transient digitizers.

Areas of biological interest where such fast time scales are important are the end use of photon energy in photosynthesis, the mechanisms of vision, electron transfer reactions, the transport of oxygen and $\mathrm{CO}_{2}$ used in metabolic processes, and structural or configurational changes by isomeric transformations induced by sudden energy additions.

The photosynthetic process is under intensive study in order to understand the various mechanisms by which photons are harvested by antenna fields, the energy transport to the reaction center, and the energy use in photosynthesis. An example is photosynthetic bacteria which absorb photon energy in carotenoid proteins to produce singlet state excitation which is transferred by singlet-to-singlet excitation to the reaction center of bacteria chlorophyll. A separation of charge would then exist across a transmembrane of the chlorophyl1-prote in complex. The charge separation then drives proton transport across the membrane which powers the photosynthetic process. The reaction kinetics, which is extremely complex, is studied by the bleaching rates and recovery time for different wavelengths, using double-pulse, femtosecond laser systems. Since some of the electron transfer times have been shown to be less than $50 \mathrm{fs}$, the development of ultrashort pulse lasers is very important. Studies include attempts to develop synthetic photosynthesis for energy production. Rhodops in found in the rods of the retina and three similar compounds found in the cones form the reaction centers for vision. The simplest to separate and use is rhodopsin which also occurs in purple bacteria. In studies of bacterial rhodopsin, the initiation of proton and ion transport across a membrane -- which is essential for biological activity -. is shown to result from structural and conformational changes caused by light absorption in which a trans- to cis-isomeric transition takes place in a few picoseconds. Much has been learned about vision mechanisms by such studies.

Subpicosecond absorption spectroscopy is used to study the interaction of hemaproteins with $\mathrm{O}_{2}$, NO, and $\mathrm{CO}$ in which it is found that the photodissociation of the ligand from the hemoprotein occurs in less than $250 \mathrm{fs}$, while the recombination occurs on the subnanosecond timescale for $\mathrm{O}_{2}$ and NO. Femtosecond IR spectroscopy is used to observe directly ligands trapped in the protein. Subpicosecond resonance Raman and IR spectroscopy is used to monitor structural changes in the heme after photodissociation and to study ligand dynamics. Prior to the advent of current short-pulse laser methods, it was necessary to carry out low temperature experiments which showed there are conformational substates, each with a different barrier height, of the photodissociated hemo-protein which controls the rate of 1 igand bonding. At higher temperature, all the substates are sampled to give an average reaction rate.

Picosecond studies have been made on internal motion in DNA molecules, using laser-excited fluorescence and streak camera detectors. The ultrashort (ca. 13 ps) excited state lifetimes of the purine and pyrimidine bases in DNA indicate that nonradiative decay dominates over energy loss by fluorescence.

Most of the DOE-sponsored work in fast chemical kinetics for biology is supported in the Chemical Division of the Basic Energy Sciences Office. The supported work is quite substantial in photosynthesis, mechanisms of vision and heme-protein, and porphryn dynamics in oxygen and carbon dioxide transport. While the area of structural changes and excited state chemistry using laser excitation have received somewhat less emphasis, the latter area should be important in studies of radiation effects on biological systems, particularly with 
regard to possible prophylaxis against excessive exposure to radiation.

Although the structure of the chemical bond has been well characterized in a variety of systems, there is a clear need to understand the dynamics of transition states in chemical reactions. Achieving this goal will help answer questions about how enzymes work and will contribute to making enzyme engineering a reality.

Transition states in chemical reactions can now be analyzed experimentally using femtosecond transition state spectroscopy (FTS). This is a technique for observing reaction fragments on their way to becoming products (Dantus et al. 1987). For a dissociation reaction, a femtosecond laser pulse is used to excite the reactant to an energy level above that needed for bond dissociation. A second collinear laser pulse is used to search for absorption by one of the reaction products. The time delay between the excitation pulse and the second (probe) pulse determines the duration of the bond breaking event. The FTS method has been used recently (Rosker et al. 1988) to measure the dissociation of ICN into $I$ and $C N$ at $205 \pm 30 \mathrm{fs}$. Theoretical work on transition-state dynamics has also moved ahead rapidly.

The principle governing the interactions of enzymes and their substrates is now well established (Kraut 1988). The principle of transition-state stabilization asserts that an enzyme binds most strongly to the reactants in their activated transition-state geometry. This significantly increases the concentration of the transition state and drives the reaction forward. The transition-state stabilization principle has been used to assist in the development of a model in two zinc proteases. Carboxypeptidase and thermolysin are zinc proteases that have very different amino acid sequences and three-dimensional structures. Yet they appear to have a common transition-state geometry. It is likely that numerous other enzymes will be found to have similar transition-state geometries. As more of these are understood, enzyme engineering will become more than a dream. Other model developments are leading to a deeper understanding of basic photobiological processes.

Certain aromatic compounds have bimodal fluorescence emission spectra. The bimodality has been explained by recent models in terms of the twisting of single and double bonds in excited-state molecules (Rettig 1986). These models have increased our understanding of the photophysical behavior of many organic, inorganic, and biologically relevant compounds. Continued research in these areas is warranted.

Excited states of molecules of all types have well defined lifetimes and decay functions that span a range of 12 decades in time. By means of laser excitation, these excited states can be used to clock the dynamics of the interaction of a fluorophore with its environment. The fluorescence measurement can al so provide information on orientational changes, collisional effects, energy and electron transfer, and the rates of chemical reactions specific to a particular location of the biomolecule. The major ways of measuring fluorescence decays involve synchronously pumped dye lasers, streak cameras, or nonlinear optical (upconversion) techniques, depending on the required time resolution.

A great deal has been learned about fast chemical kinetics using the methods discussed here. Advances in technology can be expected to open many new areas for research and to lead to fundamental understanding of the kinetics of chemical reactions.

\subsubsection{Flow Cytometry}

Flow cytometry (FCM) is a laser-based method for making rapid multivariate measurements on biological cells (Steinkamp 1984). In a flow cytometer, one or more laser beams are focused into a flow chamber containing a sample stream of biological cells surrounded by a cell-free sheath. The sample stream is a suspension of biological cells that may be stained with 
fluorescent dyes which are stoichiometric with respect to various subcellular organelles or surface antibodies. The sheath and sample streams pass through a 50 to $100 \mu \mathrm{m}$ diameter orifice and into the optical measurement region of the flow chamber. The orifice constricts the sample stream to a diameter of around $10 \mu \mathrm{m}$ so that the cells pass through the laser beams one at a time at rates of 1000 to 5000 per second. As the sample and sheath streams leave the flow chamber, a piezoelectric crystal causes the stream to break up into uniform droplets, some of which contain cells. Droplets containing cells which have given rise to specific signals can be charged and electrostatically deflected onto slides or into special containers for further analysis. This is the process of cell sorting. Multiple laser beams are focused along the sample stream at different points within the optical measurement region. Flow cytometry has been a major user of large frame gas lasers.

Typical flow cytometers have one or two cw gas lasers. One of these lasers is usually an argon ion laser with a total power output of 5 to $10 \mathrm{H}$. Typical laser lines used are a group in the UV near $360 \mathrm{~nm}, 457 \mathrm{~nm}, 488 \mathrm{~nm}$, and $514 \mathrm{~nm}$. Other gas lasers used are krypton ion $(413 \mathrm{~nm}, 531 \mathrm{~nm}, 568 \mathrm{~nm}, 647 \mathrm{~nm}$, and $753 \mathrm{~nm}$ ), hel ium-cadmium (325 $\mathrm{nm}$ and $442 \mathrm{~nm})$, and helium-neon (633 nm). An argon- or krypton-pumped dye laser is used when excitation wavelengths are needed that fall between the laser lines. Mercury arc lamps are al so sometimes used on smaller flow cytometers.

The most common measurement made with a flow cytometer is the DNA content of a mammalian cell (Merkel et al. 1987; Barlogie et al. 1980). In normal cells, DNA content is precisely regulated as cells grow through the cell cycle and divide. In cells perturbed by mutagens or that have progressed to cancer, the DNA content may no longer be euploid. This aneuploid state is readily detected and quantitated by FCM measurements (Andreeff et al. 1980). Common laser wavelengths used for these measurements are $360 \mathrm{~nm}, 457 \mathrm{~nm}$, or $488 \mathrm{~nm}$. Bacterial growth, the effects of antibacterial agents, and sometimes bacterial identification can be done with FCM (Bailey et al. 1977; Bercovier et al. 1987; Boye et al. 1983), using DNA content measurements.

Laser-based flow cytometry has had a major impact on the study of the immune system. Lymphocytes can be distinguished from other formed elements in the blood using combined forward (parallel to the incident laser beam) and $90^{\circ}$ light scattering from cells in a flow cytometer (Salzman 1982). With the lymphocytes selected by two-parameter light scattering, fluorescent monoclonal antibodies to various lymphocyte subsets can be used to identify the subsets (Hoffman et al. 1980). FCM is the major research tool for modern immunology. Continued development of FCM technology is likely to provide more new approaches to studies of the immune system.

The time dependence of the effects of physical or chemical agents on biological cells can be studied using FCM. The time at which a cell is analyzed in a flow cytometer can be measured as part of the multiparameter data (Martin and Swartzendruber 1980). Enzyme kinetics and drug transport in cells have been studied, using time as a parameter in FCM measurements. FCM is being used in studies involving transmembrane signaling in cells (Lazzari et a1. 1986). Other functional studies have involved calcium ion concentration (Rabinovitch et al. 1986) and intracellular pH measurements (Bassoe et a1. 1983). Flow cytometers are being used for preparation sorting of human chromosomes as part of the human genome initiative (Gray et al. 1987). They have also been used to detect specific DNA sequences (Trask et al. 1985).

FCM technology is tightly coupled to research into new types of lasers and photodetectors. Research support of these technologies has had a broad and beneficial impact on biological research and on human health care.

A laser technique for the rapid sequencing of large fragments [ -40 kilobases (kb)] of DNA based upon the detection of single, fluorescently tagged nucleotides cleaved from a single DNA fragment has been proposed. The 
projected rate of sequencing is -1000 bases per second. The technique is based upon the projected ability to detect single chromophores by laser-inducedfluorescence in flowing sample streams (Nguyen et a1. 1987a, 1987b). The technique involves (a) labeling the nucleotides with base-specific tags suitable for fluorescence detection, (b) selecting a desired fragment of DNA, (c) suspending the single DNA fragment in a flowing sample stream, (d) sequentially cleaving labeled bases from the free end of the DNA fragment using an exonuclease, and (e) detecting and identifying the cleaved, labeled bases as they flow through a focused laser beam. The rate that bases can be sequenced is determined by the kinetics of the exonuclease cleavage reaction and the time required for detection and identification of the labeled bases. Based upon results for the detection of rhodamine-6G18, the investigators anticipate sequencing rates of -1000 bases per second on a single strand of DNA several tens of kilobases (kb) in length. In order to detect true single molecules, it is necessary to improve the sensitivity for fluorescence detection. With improvements in the apparatus, the investigators envision single molecule detection with excellent signal-to-noise ratio.

\subsubsection{Biosensors}

Fiber sensors find their greatest use in monitoring clinically and biochemically important analytes such as serum electrolytes, metabolites, co-enzymes, immunoproteins, and inhibitors. Biosensors are available which respond selectively and reversibly to the concentration or activity of chemical species in biological samples. In some cases, a biologically active material is incorporated to make intimate contact with an appropriate transduction element for detecting, reversibly and selectively, a chemical species in any type of sample (Arnold and Meyerhoff 1988). New breakthroughs in optical sensor technology are likely, and this is an area of potential importance to several of the OHER programs. Potential biomedical sensor applications include critical care (in vivo determination of $\mathrm{O}_{2}, \mathrm{CO}_{2}, \mathrm{pH}$, temperature, $\mathrm{K}^{+}$), chronic maintenance (in vivo and in vitro determinations of glucose, therapeutic drugs), and acute diagnosis (in vitro studies of blood chemistry, abnormal function, infection, diagnostic metabolites) (Wolfbeis 1987). Laser-induced fluorescence is considered to be one of the most promising approaches in this field because of its detection sensitivity and versatility. For example, with fiber optic sensors based on fluorescence, in situ determination of immunoglobulin, IgG, and benzo[a]pyrene was performed with detection sensitivities of $25 \mathrm{fmol}$ and 1 fmol, respectively. Among the bioanalytical applications of the fluorescence technique (Bright 1988), one includes (a) assays directed at the determination of DNA and RNA; (b) enzymatic methods, e.g., for the determination of glucose, sucrose, and fructose and for the evaluation of kidney function; (c) studies of protein-ligand interactions, e.g., the understanding of the stoichiometry and affinity of protein-binding sites is of critical importance in elucidating protein functions and dynamics; and (d) fluoroimmunoassays (FIA). This last concept deserves special attention. In its simplest form, it involves a competitive binding reaction between an antigen ( $\mathrm{Ag}$ the target analyte), a labeled antigen $\left(\mathrm{Ag}^{\circ}\right)$, and a highly selective antibody (Ab) to form $A g A b$ and $\mathrm{Ag}^{\circ} \mathrm{Ab}$. Basically, the immunoassays exploit some difference between the free labeled antigen $\left(\mathrm{Ag}^{*}\right)$, and the antibody-bound labeled antigen $\left(A g^{\circ} A b\right)$ to quantify the target analyte. FIA is expected to be more sensitive than is radioimmunoassay (RIA), where radioisotopes are used as labels.

Polarization-based fluoroimmunoassay (PFIA) and fluorescence anisotropy selective technique - fluoroimmunoassay (FAST-FIA) constitute attractive detection methods in FIA. In the first case (PFIA) developed for many drugs, antibiotics, and steroids, the fluorescent label can be used to track 
rotational diffusion which manifests itself in a large polarization for a larger species versus a small species. Thus $\mathrm{Ag}^{\circ}$ and $\mathrm{Ag}{ }^{\circ} \mathrm{Ab}$ are easily distinguishable by measuring the fluorescence polarization. In the last detection method (FAST-FIA) applied to the determination of bovine serum albumin, the individual rotational rates of the molecular species $\mathrm{Ag}^{\circ}$ and $\mathrm{Ag}^{\circ} \mathrm{Ab}$ could be directly resolved.

Time-resolved fluorescence (TRF) is a key technique for discriminating against background fluorescence which can be due to other matrix components. For example, fluorescein in serum-containing samples suffers from interference of bilirubin because of spectrally overlapping emissions. However, fluorescein and bilirubin decay times are about $4 \mathrm{~ns}$ and $0.1 \mathrm{~ns}$, respectively, so that time gating can be successfully used.

Rare earth chelates are of special interest. FIA with lanthanide labels is a fast moving research field and is likely to grow in the future (Soini and Lövgren 1987). A directly fluorescent lanthanide label can be used in which both the fluorescent and the chelating properties are incorporated into one Tigand-lanthanide complex. When such complex is bound to an immunoreactive component, completely new assay designs based on TRF become real. In other cases, the lanthanide is bound to one of the immunocomponents in an essentially nonfluorescent form. After completion of the immunoreaction, the lanthanide is dissociated from the immunocomponent and brought into solution, where a highly fluorescent chelate is formed. This technique is called dissociation enhanced fluoroimmunoassay.

Presently, fiber optic sensors have some limitations, associated mainly with matrix effects. Nevertheless, the future of fiber optic sensors is extremely promising. The ultimate goal would be the development of real time, in vivo chemical and immunochemical sensors of the determination at trace and ultratrace levels of specific biomolecules and bioactive species, with simple sensors taking over essentially any optical measurement that one can make in a cuvette.

\subsubsection{U7trasensitive Detection and Analysis for Biological Research}

Many biological research activities require the detection and analysis of micro-quantities of biological materials. Examples include FCM, cytochemical analyses of single cells, detecting trace chemicals or particulates of health and environmental concern, measuring cellular uptake of chemicals, and analyzing microquantities of proteins or nucleic acids via spectrophotometry, chromatography, or e]ectrophoresis.

Several laser-based technologies could usefully be considered for these applications. Zeeman interferometry (Johnston 1987, 1989; Johnston et al. 1988) is a relatively new technique that looks particularly promising. It exploits the two-frequency, Zeeman effect laser for simple and high resolution interferometry. Many of the stability and complexity problems that plague conventional interferometric or optical heterodyne techniques are not present (Johnston 1987, 1989; Johnston et al. 1988). A suitably developed Zeeman interferometer could be used as an ultrasensitive refractive index detector for chromatography or electrophoresis. The Zeeman interferometer is substantially superior to conventional refractive index detectors in its sensitivity, inherent absence of $\mathrm{drift}$, and its ability to be applied to extremely small volumes. Micro-quantities of proteins or nucleic acids could be detected in real time as they are eluted from chromatography or electrophoresis columns, without the need for fluorescent or radioactive tags or the damaging effects of UV radiation.

Photothermal spectroscopy (Harris 1982; Dovichi 1988) is another laser-based technology that warrants consideration. The technique measures the small temperature rise (or refractive index change) in a sample when it absorbs small amounts of incident 7 ight (Johnston 1987; Harris 1982; Dovichi 1988). Zeeman 
interferometry may again play an important role in photothermal applications (Johnston 1987, 1989; Johnston et al. 1988).

Photothermal spectroscopy has many experimental advantages (simplicity, sensitivity, relative freedom from model dependence) over photoacoustic spectroscopy and can be better applied to biological samples and small volumes. Photothermal spectroscopy can be used for microchemical analyses (on small volumes or on large volumes with trace contaminants) and for linear or circular dichroism measurements.

Surprisingly, dichroism applications of photothermal spectroscopy have received little attention. They are, however, of great potential value for studying trace samples and for dichroism measurements at very short wavelengths. The latter are of much structural interest, but are difficult due to the strong absorption of water and the shortage of suitable light sources at wavelengths below $180 \mathrm{~nm}$ (Williams et a1. 1986).

Light scattering has always been a powerful tool in biological studies. In recent years, there has been increased interest in the polarization properties of scattered light (see, for example, Johnston et a1. 1988; Bustamante et al. 1982). This is evidenced by measurements of the Mueller scattering matrix and amplitude scattering matrix for biological samples and by polarized Raman scattering experiments. These measurements are all experimentally difficult, yet should be encouraged because of the large amount of structural information that is theoretically available. $A$ third area, polarized dynamic light scattering, may not have received the attention it deserves. Autocorrelation (unpolarized) scattering measurements have proven to be useful for understanding translational and rotational diffusion coefficients (Berne and Pecora 1985). Polarized dynamic scattering measurements have the potential for providing important information on structural and conformational fluctuations in biological macromolecules. Such information is, for example, of great importance in understanding protein thermodynamics, biopolymer chemistry, and protein-drug interactions.

Finally, experimental advances in pulsed-laser technology make it practical to study the elastic and Raman scattering of femtosecond laser pulses from biological cells (Chang and Chang 1988; Biswas and Chylek 1988). Both polarized and unpolarized measurements are of interest. Such measurements can provide additional size and compositional and structural information about a cell not readily accessible from steady-state scattering measurements.

5.4.6. Structural Analysis and Sequencing of Biomolecules

Structural analysis and sequencing of nucleic acids and peptides (Landegren et al. 1988; Maugh 1984; Garnick et al. 1988) will be a key accomplishment in biological research over the next decade. The ability to rapidly determine DNA sequences would facilitate the identification of disease-associated genes which cause inherited disorders such as cystic fibrosis, phenylketonuria and Huntington's disease. In some of these illnesses, large deletions of sequences in the genes may occur or just a single nucleotide substitution may be responsible for the disorder. Nucleic acid sequencing is also important for determining damage to the DNA due to mutagenesis from external agents such as chemicals or radionuclides. Peptide sequencing and identification is an equally important problem for studying critical metabolic disorders in the body and for quality control in the biotechnology industry (Garnick et al. 1988).

Both the peptide-protein and nucleic acid sequencing problems are complicated by the large number of units to be sequenced. This is especially true in the nucleic acid sequencing problem where there are $>10^{\circ}$ base pairs in DNA. Some DNA sequencing techniques have been automated (Landegren et al. 1988; Maugh 1984; Garnick et a1. 1988); however, 
these are too slow and are prone to a number of errors. Also, these techniques fail when there are a large number of modified or mutant nucleosides as in RNA, for example (McCloskey and Nishimura 1977). In order to identify uniquely each of the four base units and to distinguish them from the modified or mutant nucleosides that may be present, a technique such as mass spectrometry or laser spectroscopy is required. Fluorescent tags can be used to identify each of the four regular base pairs, but cannot easily identify mutant or modified species in the sequence. Mass spectrometry is universal in that any substituted unit can be identified.

To accomplish sequencing rapidly, continuous flow techniques need to be developed (Covey et al. 1986). This would involve biochemical degradation of a nucleic acid strand followed by a subsequent continuous injection of the effluent into a mass spectrometer. Continuous flow FAB (Caprioli et al. 1986) has proven to be valuable in the sequencing of peptides. As solutions are injected into the $M S, F A B$ is used to vaporize and ionize the peptides for identification. Techniques such as laser-induced MPI may be interfaced to this method for enhanced selectivity against the liquid matrix which often provides background interference in FAB-MS. Laser-induced MPI can also provide versatility in identification based upon induced soft ionization or extensive fragmentation.

Light microprobe techniques (Kopelman and Lewis, unpublished) might be a second possible method for DNA sequencing. Each different nucleic acid base would be labeled with a different fluorescent probe. If a light source that could be confined to $.5 \mathrm{~nm}$ (the dimension of the nucleoside plus fluorescent probe) were developed, then the light probe could be scanned along the intact nucleic acid strand to obtain sequence information. This procedure would obviate the problems of chemical degradation, but would be accurate if only the four usual bases were present. Development of a $.5 \mathrm{~nm}$ light source would be the main problem since light in the near UV and visible would be diffraction limited by several hundred namometers. However, recent work by Kopelman (Michigan) and Lewis (Jerusalem) has utilized the properties of exciton migration to transfer UV light energy to a target fluorophore in contact with the crystal in a region $<100 \mathrm{~nm}$.

\subsubsection{Genome Sequencing}

Laser technology could have a direct and impressive impact on genome sequencing. Several approaches are being taken, including various imaging methodologies. In order to sequence the base units along a DNA segment, a spatial resolution of $1 \mathrm{~nm}$ or better is required as well as a tagging scheme to distinguish the base units from each other. Several methodologies are being explored, including coherent electron microscopy, acoustic imaging, and scanning tunneling microscopy which may be able to provide the desired resolution.

One very promising method for sequencing may be $X$-ray microholography. This novel $X$-ray technique, under development by a research group at the University of 111 inois at Chicago (Boyer 1989), is performed by tagging each type of base unit with a separate atomic species. The prepared tagged DNA segment is exposed to a specific $X$-ray radiation wavelength from a synchrotron light source or an X-ray laser, corresponding to a resonance (near an absorption edge) of each atomic tag labelling each base unit. By using an X-ray CCD for detection, it is estimated that a Fourier transform hologram could be obtained with $1 \mathrm{~nm}$ resolution with a $200 \mathrm{~s}$ exposure time for each type of base unit of a 100,000 string. A reconstruction algorithm has been developed which permits a computer-generated video image to be obtained. Optical reconstruction techniques have also been developed. Superimposing four video images for each base type at the appropriate wavelength would provide the sequence for the DNA string at an overall rate of 100 base units per second or greater. Such a rate is essential if DNA sequencing is to 
become a general tool for biology and medicine. The DNA string has to be fossilized and contained within supporting films (probably diamond) to limit the effect of radiation damage from the synchrotron beam so that the relative positions of the markers are not affected. The use of a short-pulse, Xray laser would eliminate the damage problem and the necessity of protecting the DNA string.

other interesting methods for genome sequencing include light microprobe techniques (Kopelman 1989). This method involves exciton migration and fluorescence in solid-state materials. This technique can be coupled with micropipette technology to confine energy to a very small ( $<100 \mathrm{~nm})$ spatial volume from which the energy can be transferred directly to another species. If this technique can be further developed, such that energy transfer can be confined to a probe tip of $<1 \mathrm{~nm}$, the required resolution would be available for distinguishing tagged fluorescent probes specific to each base in a long DNA strand. The probe could be scanned rapidly along the DNA molecule to obtain the specific sequence.

One of the most powerful methods available for sequencing is analysis via mass spectrometry, especially when a large number of modified or mutant nucleosides are present, as, for example, in RNA (MCCloskey and Nishimura 1977). In this case, positive mass identification of the nucleic acid components is required. The problem of rapidly sequencing a large number of nucleic acid base pairs still remains. One developing trend in mass spectrometry is the use of exonucleases to sequentially break down the DNA strand followed by continuous flow injection methods into a MS. As each base pair is injected into the MS device, it can be sequenced rapidly and accurately. Current research is actively engaged in continuous liquid injection methods interfaced to various MS ionization sources. A relatively new method which has proven to be valuable in the sequencing of peptides and oligonucleotides is continuous flow $F A B$ (Caprioli et al. 1986). In this method, a mixture of peptides is separated by microbore HPLC and the eluted product mixed with glycerol. As the solution is injected into the MS, FAB is used to vaporize and ionize the peptides for identification. Techniques such as 1aser-induced MPI may be interfaced to this method for enhanced selectivity against the liquid matrix which often provides background interference in FABMS. Laser-induced MPI can also provide versatility in identification based upon induced soft ionization or extensive fragmentation. Using high repetition rate excimer lasers $(>100 \mathrm{~Hz})$, the main limitation for speed in sequencing DNA strands will be the degradation and introduction processes.

\subsection{NUCLEAR MEDICINE USING STABLE ISOTOPES}

Isotopes have enormously important roles to play in the diagnosis and treatment of human diseases. The basic idea is to attach these isotopes to such diverse tags as neuroreceptors and monoclonal antibodies and to use the characteristic radiation emitted from these isotopes to trace their flow within the human organism. Radionuclides are by far the most commonly found agents employed in these medical applications since their location may be directly imaged. There is currently an important thrust within DOE, for example, to develop radiopharmaceuticals including monoclonal antibodies for improved methods of diagnosis and treatment. This strategy may assist the early detection of cancer, for following the response of patients to treatment, and for general cancer therapy. Radionuclides themselves, however, pose a significant health threat to the patient.

With the advent of advanced laser spectroscopic techniques, it appears feasible to find a number of complementary approaches to utilize stable isotopes to examine these processes, completely eliminating the risks of radiation. There are currently few examples of the use of stable 
isotopes for these medical applications. These tags are not as convenient to trace through the body since they must be examined from blood, urine, or other samples extracted from the body. The ultralow detection limits and the isotopic selectivity of laser-based analytical schemes, however, offers potential for major new clinical approaches.

A critical issue among health physicists is to provide rapid, low-cost methods for the detection of uranium and transuranic species in biological samples. Currently, uranium or plutonium analyses in urine require extensive sample pretreatment, long counting times, or an intense source of neutrons. In a series of preliminary experiments (Gerke et al. 1988), it has been possible to volatilize uranium atoms directly from a urine sample and to collisionally ionize the atom after 2-photon resonant excitation to a Rydberg level using inexpensive $\mathrm{N}_{2}$-pumped dye lasers. The experiments were performed at low pressure (1 to 10 torr), and the ions could be counted with high efficiency using just a thermionic diode. Detection limits in the few femtogram range have been demonstrated for calcium, and these 7 imits appear feasible for the actinides. Much research into this promising approach is needed to improve isotopic selectivity, sampling methods, and laser excitation schemes. If these approaches could be generalized, wide screening of patients for a wide variety of stable isotopes may be feasible.

Sampling is a continual problem for any biological assay involving heavy atoms. Converting the target isotope quantitatively into the gas phase with high efficiency from a variety of matrices is a topic of intense research interest. The methods which utilize laser desorption or $\mathrm{keV}$ ion-desorption from a complex solid matrix are particularly attractive since there is efficient temporal and spacial overlap between the atomized sample and the laser ionization probe which makes it possible to use smaller samples. Further, with RIS the problem of interferences is greatly reduced, allowing more quantitative schemes to be easily developed for many stable isotopes. Recently (Moore et al. 1987) this method has been used to determine the copper and molybdenum concentrations in microliter samples of blood by an isotope dilution procedure. The present accuracy of $15 \%$ at the femtogram range suggests this approach may be important in following isotopic enrichments in small samples for metabolism studies.

As research into the laser detection of stable isotopes continues to increase in sophistication, there are many new avenues of application which may open. Direct analysis of inhaleable particulates may provide early warnings of toxic dust. The role of trace metals in nutrition, toxicity, and disease etiology could be more clearly evaluated.

\subsection{RADIATION DOSIMETRY}

Laser technology and spectroscopy make it attractive to consider new methods for the solution of old problems in radiation dosimetry. Actually, laser technology has already been used to make more convenient systems for gamma ray dosimetry. Thermoluminescent dosimeters are used to assess the exposure of personnel who wear these devices. After exposure the small samples are heated with a laser beam to initiate fluorescence from the dosimeter. Here we explore laser spectroscopy and speculate on more novel approaches by considering two examples. The first example is appropriate for new research in radiation dosimetry based on the use of resonance ionization spectroscopy. When ionizing radiation of any type ( $X$ rays, gamma rays, neutrons, etc.) interact with matter, both excited states and ion pairs are created and in about equal numbers. However, nearly all systems of radiation dosimetry, and even the most sophisticated of detectors like those used in particle physics, measure the ionization created and neglect the rich amount of information which could be retrieved from a study of the excited species.

In radiation dosimetry, even in the most advanced form such as microdosimetry, the entire emphas is is on 
the creation of ion pairs. However, it is usually assumed that when charged particles interact with matter, e.g., in a gas, the particle loses as much energy in creating excited states as in ion pairs. Further, in most theories of charged particle interaction, the assumption is made that the excited state population compared to the number of ion pairs is independent of the energy of the primary charged particle or the secondary particles created. These assumptions could be tested in actual experiments by using the techniques of laser spectroscopy.

We note that, historically, the RIS method was invented for just these studies on excited states. However, the demand for RIS in analytical chemistry to detect atoms and molecules in their ground states diverted attention from these studies. There is considerable evidence that the study of excited states would yield new information. Certainly, as electrons slow down in matter, they reach an energy where low-lying metastable states become more important than other types of excited states or ion pairs. To the extreme, it is noted that the magnetic monopole (if it exists according to current grand unification theories) would interact, for example, in helium gas to create only metastable states. In research where attempts are made to correlate track structure with the effects of radiation on biological cells, it is appropriate to consider the role of excited states.

Another example where the quantum state selectivity of RIS could be used is found in neutron dosimetry (Hurst et al. 1987). Workers at power reactors are possibly exposed to neutrons in an energy range (about 10 to $100 \mathrm{keV}$ ) which cannot be detected with any presently available personnel badge. It turns out, however, that charged particles sputter atoms from a solid target, with the cross section for the process peaking in the few keV region. This process could be incorporated into a neutron badge to give just the desired response in the energy region that is most critical for the power reactors. The ability to measure small numbers of these sputtered atoms is provided with RIS. For instance, it has been shown that the incorporation of xenon atoms into amorphous metal alloys should lead to a personnel dosimeter which would have excellent energy dependence from about $1 \mathrm{keV}$ to about $20 \mathrm{MeV}$ and would be so sensitive that a few mrad of exposure could be reliably recorded. In practice, amorphous metal alloys containing xenon would be ground into fine particles and enclosed in a smallenvelope which would retain the sputtered xenon atoms.

The above examples indicate that methods of laser spectroscopy could be used for more definitive research on the interaction of radiation with matter, and there is every reason to believe that such research would Jead to improved practices in personnel dosimetry, even in the near term.

\section{REFERENCES}

Andreeff, M., Z. Darzynkiewicz, T. K. Sharpless, B. D. Clarkson, and M. R. Melamed (1980) Blood 55, 282-293.

Arnold, M. A. (1985) Anal. Chem. 57, 565.

Arnold, M. A. and M. E. Meyerhoff (1988) CRC Crit. Rev. Anal. Chem. 20, 149.

Bailey, J. E., J. Fazel-Madjlessi, D. N. Quitty et a1. (1977) Science 198, 1175.

Barlogie, B., B. Drewinko, J. Schumann et a1. (1.980) Amer. J. Med. 69, 195.

Bassoe, C. F., 0. D. Laerum, J. Glette et al. (1983) Cytometry 4, 254.

Bercovier, H., M. Resnick, D. Kornitzer, and L. Levy (1987) J. Microbiol. Methods I, 167 .

Berne, B. and R. Pecora (1985) DYNAMIC LIGHT SCATTERING (Plenum Press, New York, NY).

Biswas, A. and P. Chylek (1988) Appl. Phys. Lett. $\underline{52,1642 .}$ 
Boye, E., H. B. Steen, and K. Skarstad (1983) J. Gen. Microbiol. 129, 973.

Boyer, Prof. Keith, University of Illinois-Chicago (1989) personal communication.

Braman, R. S. (1983) in Analytical Aspects of Environmental Chemistry, Vol. B758F, Chapter 1.

Bright, F. W. (1988) Anal. Chem. 60, 1031A.

Bushaw, B. A., B. D. Cannon, G. K. Gerke, and T. J. Whitaker (1987) in RESONANCE IONIZATION SPECTROSCOPY 1986, edited by G. S. Hurst and C. G. Morgan (Institute of Physics, Bristol, U.K.), pp. 103-108.

Bushaw, B. A. and G. K. Gerke (1988) in RESONANCE IONIZATION SPECTROSCOPY 1988, edited by T. B. Lucatorto and J. E. Parks (Institute of Physics, Bristol, U.K.), pp. 277-280.

Bustamante C., I. Tinoco, and M. F. Maestre (1982) J. Chem. Phys. 76, 3440.

Butler, J. D. (1979) AIR POLLUTION CHEMISTRY (Academic Press, New York).

Caprioli, R. M., T. Fan, and J. S. Cottrel1 (1986) Anal. Chem. 58, 2949.

Chang, R. K. and J. Z. Chang (1988) Opt. Lett. 13, 270.

Chappelle, E. W. and D. L. Williams (1986) in PROCEEDINGS OF THE IGARSS'86 SYMPOSIUM (ESA Publications Division ESA254), p. 1591.

Cooper, R. S., R. Jankowiak, J. M. Hayes, L. Pei-qui, and G. M. Smal1 (1988) Anal. Chem. 60, 2692.

Covey, T. R., E. D. Lee, A. P. Bruins, and J. D. Henion (1986) Anal. Chem. 58, $1451 \mathrm{~A}$.

Cremers, David A. and Leon J.Radziemski (1987) in LASER SPECTROSCOPY AND ITS APPLICATIONS, edited by Leon $J$. Radziemski, Richard $W$. Solarz, and
Jeffrey A. Paisner (Marcel Dekker, New York), pp. 351-415.

Dantus, M., M. J. Rosker, and A. H. Zewail (1987) J. Chem. Phys. 87, 2395.

Dovichi, N. J. (1988) Prog. Anal. Spectrosc. 11, 179.

Fairbank, W. M. Jr., R. D. LaBelle, R. A. Keller, C. M. Miller, J. Poths (1988) and B. L. Fearey, in RESONANCE IONIZATION SPECTROSCOPY 1988, edited by T. B. Lucatorto and J. E. Parks (Institute of Physics, Bristol, U.K.), pp. 53-56.

Garnick, R. L., N. J. Solli, and P. A. Papa (1988) Anal. Chem. 60, 2546-2557.

Gerke, G. K., B. A. Bushaw, and T. J. Whitaker (1988) in RESONANCE IONIZATION SPECTROSCOPY 1988, edited by T. B. Lucatorto and J. E. Parks (Conf. Series No. 95, Institute of Physics, Bristol, U.K.), p. 311 .

Goldman, D. S. (November 1988) American Laboratory. Gray, J. W., P. N. Dean, J. C. Fuscoe, D. C. Peters, B. J. Trask, G. J. van den Engh, and M. A. Van Dilla (1987) Science 238, 323.

Hardis, J. E., W. R. Peifer, C. L. Cromer, A. L. Migdall, and A. C. Parr (1988) in RESONANCE IONIZATION SPECTROSCOPY 1988, edited by $T$. B. Lucatorto and J. E. Parks (Institute of Physics, Bristol, U.K.), 237-240.

Harris, T. D. (1982) Anal. Chem. 54, $741 \mathrm{~A}$.

Hoffman, R. A., P. C. Kung, P. Hansen et a) (1980) Proc. Nat. Acad. Sci. USA 77, 4914 .

Hoge, F. E., R. N. Swift, and J. K. Yungel (1983) Appl. Opt. 22, 2991.

Houk, Robert S. (1986) in ANALYTICAL APPLICATIONS OF LASERS, edited by E. H. Piepmejer (John Wiley \& Sons, New York), pp. 588-626.

Hurst, G. S., Miroslaw Bialkowski, and D. $H$. Lowndes (1988) in RESONANCE IONIZATION 
SPECTROSCOPY 1988, edited by T. B. Lucatorto and J. E. Parks (Institute of Physics, Bristol, U.K.), pp. 229-232.

Hurst, G. S., M. G. Payne, S. D. Kramer, C. H. Chen, R. C. Phillips, S. L. Allman, G. D. Alton, and J.W.T. Dabbs (1985) Repts. Prog. Phys. 48, 1333-1370.

Hurst, G. Samuel, Harold W. Schmitt, Norbert Thonnard, and Tom J. Whitaker (1987) "Neutron Dosimeter," U.S. Patent No. $4,699,751$.

Johnston, R. G. (1987) TECHNICAL DIGEST, LASER APPLICATIONS TO CHEMICAL ANALYSIS (Optical Society of America, Washington, D.C.), pp. 136-139.

Johnston, R. G. (1989) Appl. Phys. Lett. 54, 121 .

Johnston, R. G., S. B. Singham, and G. C. Salzman (1988) Comments on Molecular and CelTular Biophysics 5,171 .

Kenny, J. E. and G. B. Jarvis (1987) Anal. Instr. 16423 .

Kim, J. I. (1986) in HANDBOOK ON THE PHYSICS AND CHEMISTRY OF THE ACTINIDES, edited by $A$. J. Freeman and C. Keller (Elsevier Science Publishers), Chapter 8, p. 413 .

Kim, H. K. and T. Ryan, Eds. (1975) "The Use of Lasers for Hydrographic Studies," NASA Report SP-375.

Kopelman, Prof. Raoul, University of Michigan (1989) personal communication.

Kopelman, R. and A. Lewis (unpublished results).

Kraut, Joseph (1988) Science 242, 533-540.

Kulp, T. J., I. Camins, S. M. Angel, C. Munkholm, and D. R. Watt (1987) Anal. Chem. 59, 2849.

Lam, Z., M. A. Comisarow, and G. G. S. Dutton (1988) Anal. Chem. 60, 2306. Landegren, V., R. Kaiser, C. T. Caskey, and L. Hood (1988) Science 242, 229-242.
Laqua, K. (1979) in ANALYTICAL LASER SPECTROSCOPY, edited by Nicolo Omenetto (John Wiley \& Sons, New York), p. 48.

Lazzari, K. G., P. J. Proton, and E. R. Simons (1986) J. Biol. Chem. 261, 9710.

Lehmann, B. E., D. F. Rauber, $N$. Thonnard, and R. D. Will is (1987) in RESONANCE IONIZATION SPECTROSCOPY 1986, edited by G. S. Hurst and C. G. Morgan (Institute of Physics, Bristol, U.K.), pp. 81-84.

Lichtenthaler, H. K. and U. Rinderle (1988) CRC Crit. Rev. Anal. Chem. 19, Supp 1 1, 529 .

Martin, J. C. and D. E. Swartzendruber (1980) Science 207, 199.

Maugh, T. H. II (1984) Science 226, 11831184.

McCloskey, J. A., and S. Nishimura (1977) Acc. Chem. Res. 10, 403.

Merkel, E., L. G. Dressler, and W. L. McGuire (1987) J. Clin. Oncol. $\underline{5}$, 1690-1703.

Moore, L. J., J. E. Parks, E. H. Taylor, D. W. Beekman, and M. T. Spaar (1987) in RESONANCE IONIZATION SPSECTROSCOPY 1986, edited by $G$. S. Hurst and C. Grey Morgan (Conf. Series No. 84, Institute of Physics, Bristol, U.K.), p. 239.

National Academy Press (1984) "Global Tropospheric Chemistry - A Plan for Action" (National Academy Press, Washington, DC).

Nguyen, D. C., R. A. Keller, J. H. Jett, and J. C. Martin (1987a) Anal. Chem. 59, 2158 .

Nguyen, D. C., R. A. Keller, and $M$. Trkula (1987b) J. Opt. Soc. Am. B 4, 138.

Niemax, K., J. Lawrenz, and A. Obrebski (1987) in RESONANCE IONIZATION SPECTROSCOPY 1986, edited by G. S. Hurst and C. G. Morgan (Institute of Physics, Bristol, U.K.), pp. 45-50. 
Nuwaysir, L. M. and C. L. Wilkens (1989) in LASERS AND MASS SPECTROMETRY, edited by D. M. Lubman (Oxford University Press, Oxford, U.K.), Chapter 13.

Payne, M. G. (1988) in RESONANCE IONIZATION SPECTROSCOPY 1988, edited by T. B. Lucatorto and J. E. Parks (Institute of Physics, Bristol, U.K.), pp. 221-224.

Piepmeier, E. H. (1986) in ANALYTICAL APPLICATIONS OF LASERS, edited by E. H. Piepmeier (John Wiley \& Sons, New York), p. 627-669.

Rabinovitch, P. S., C. H. June, A. Grossmann, and J. A. Ledbetter (1986) J. Immunol. 137, 9952.

Reddy, M. V., R. C. Gupta, E. Randernath, and K. Randernath (1984) in CARCINOGENESIS (London, U.K.), p. 231.

Rettig, Wolfgang (1986) Angew. Chem. Int. Ed. Engl. 25, 971-988.

Rosker, Mark J., Marcos Dantus, and Ahmed H. Zewail (1988) Science 241, 1200-1202.

Saari, L. A. (1987) Trends Anal. Chem. $\underline{6}$, 85.

Salzman, D. C. in CELL ANALYSIS (1982) edited by $N$. Catsimpoolas (Plenum, New York, NY).

Sanders, M. J., R. S. Cooper, R. Jankowiak, G. J. Small, V. Heisig, and A. M. Jeffrey (1986) Anal. Chem. $58,816$.
Saunders, P. A. and J. D. Wilkins (1987) Chemistry in Britain 23, 448.

Soini, E. and T. Lövgren (1987) CRC Crit. Rev. Anal. Chem. 18, 105.

Steinkamp, John A. (1984) Rev. Sci. Instrum. 55, 1375-1400.

Thonnard, N., R. D. Will is, M. C. Wright, and W. A. Davis (1987) in RESONANCE IONIZATION SPECTROSCOPY 1986, edited by G. S. Hurst and C. G. Morgan (Institute of Physics, Bristol, U.K.), pp. 75-80.

Trask, B. J., G. J. van den Engh, J. Landegent, $N$. Jansen in de Wal, and $M$. van der Ploeg (1985) Science 230, 1401.

Vodacek, A. and W. D. Philpot (1987) Remote Sens. of Environ. 21, 83.

Vo-Dinh, T. (1978) Anal. Chem. 50, 396.

Whitaker, Tom J. and Bret D. Cannon (1988) in RESONANCE IONIZATION SPECTROSCOPY 1988, edited by T. B. Lucatorto and J. E. Parks (Institute of Physics, Bristol, U.K.), pp. 225-228.

Williams, A. L., C. Cheong, and L. B. Clark (1986) Nucleic Acids Res. 14, 6649.

Wolfbeis, D. S. (1987) Pure Appl. Chem. 59, 663 .

Wolfbeis, D. S. (1986) Fresenius Z. Ana?. Chem. $325,387$. 


\section{APPENDIX I}

\section{LASER ASSESSMENT STUDY ACRONYMS}

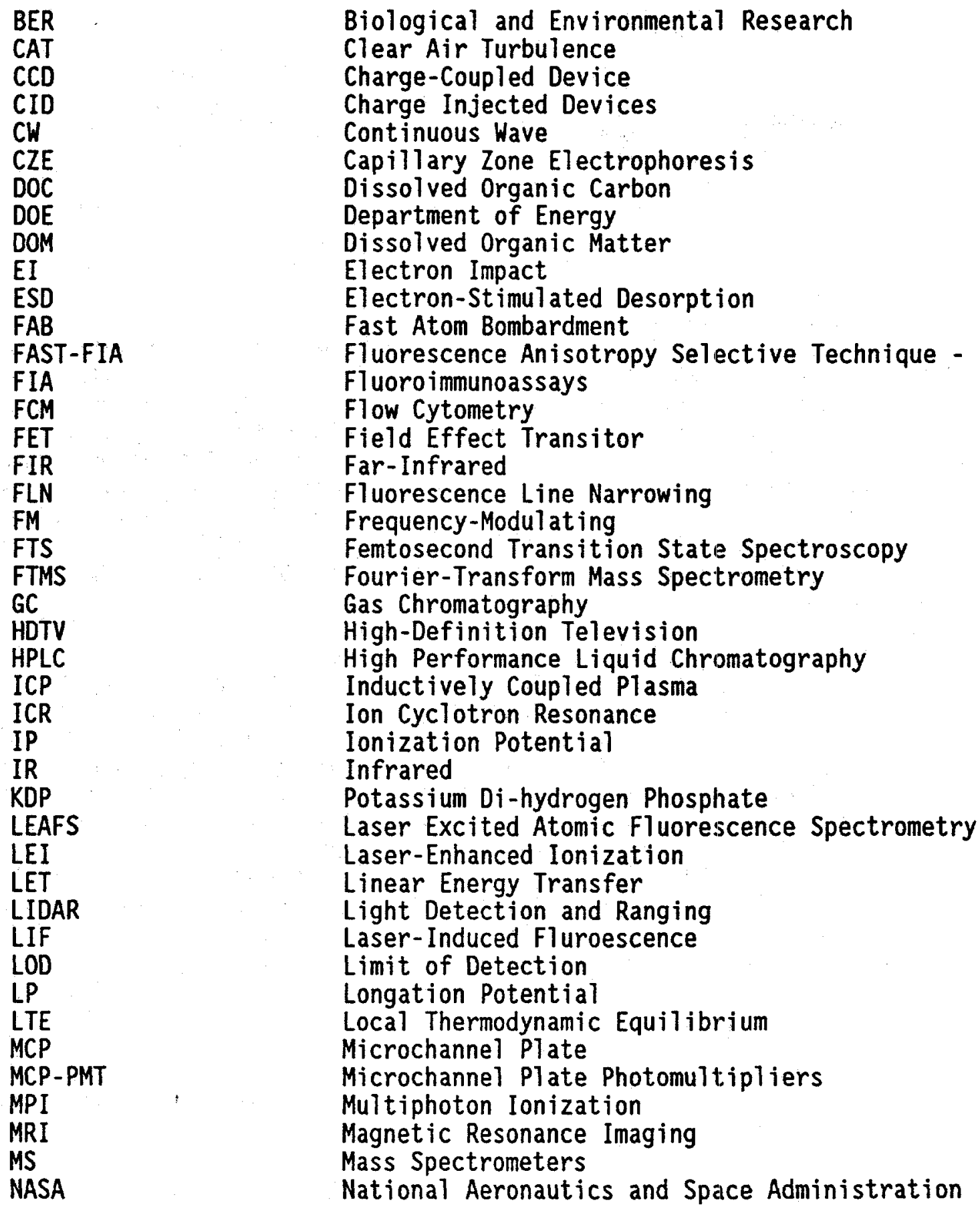


OGE

OHER

OPO

$P A$

PET

PFIA

PMT

PNAH

RBE

REMPI

RF

RFS

RIA

RIMS

RIS

SERS

SF

SIRIS

SIT

SOS

SRI

SSJ

STEM

TOF

TRF

UV

VUV

$X U X$
Optogalvanic Effect

Office of Health and Environmental Research

Optical Parametric Oscillator

Photoacoustics

Positron Emission Tomography

Polarization-based Fluoroimmunoassay

Photomultiplier

Polynuclear Aromatic Hydrocarbons

Relative Biological Effectiveness

Resonance Enhanced Multiphonic Ionization

Radio Frequency

Remote Fiber Spectroscopy

Radioimmunoassay

Resonance Ionization Mass Spectroscopy

Resonance Ionization Spectroscopy

Surface Enhanced Raman Spectroscopy

Supercritical-Fluid

Sputter-Initiated Resonance Ionization Spectroscopy

Silicon-Intensified Target

Silicon-On-Sapphire

Stanford Research Institute

Supersonic Jets

Scanning Transmission Electron Microscope

Time-of-Flight

Time-Resolved Fluorescence

UTtraviolet

Vacuum UItraviolet

Extreme U1traviolet 


\section{APPENDIX II}

\section{INTRODUCTION TO TERMINOLOGY}

LASER: The acronym laser is derived from "Light Amplification by Stimulated Emission of Radiation" and is a light source with some unique properties. Most are familiar with the directional property of a laser beam and know that a laser beam has such a small divergence that it can be used to illuminate a small spot on the moon from a point on the earth. For the purposes of this report it is even more significant that lasers are a very intense light source and that they can be monochromatic, having a single wavelength or color. A variety of laser systems are now commercially available that can be "tuned" to almost any desired color. Currently these tend to be "dye" lasers which use liquids similar to those used to dye clothing. However, these are being replaced with solid-state lasers which are much more convenient to use.

ABSORPTION: When a laser beam strikes a material, some of its energy may be absorbed by the material. As a consequence, the material may be heated or other physical processes as described below may occur.

SCATTERING: Some of the energy striking a material can be radiated away immediately or scattered from the target. In other cases (see below), this radiation can be delayed and is not usually considered to be a scattering process.

TRANSMISSION: When a radiation beam such as a laser strikes a material of finite thickness, some of the beam can pass through the material without interaction. Consistent with these definitions and the conservation of energy principle, the incident energy is equal to the sum of the absorbed energy, the scattered energy, and the transmitted energy.

EXCITATION: The energy absorbed by a material may cause the excitation of the material. Of course, the nature of this excitation depends critically on the type of material. For an atomic gas this excitation is simply the promotion of an electron in its lowest or ground state to some higher state allowed by a quantum mechanical description of the atom. For molecular gases, this excitation process is more complex; in addition to electronic excitation, there is vibrational excitation as atoms in the molecule move with respect to each other, and there is rotational excitation as the entire molecule rotates about some axis of symmetry. In condensed matter such as a liquid or a solid, collective processes--often described in terms of phonons or plasmons--add to the complexity of the excitation processes.

FLUORESCENCE: When matter in any of its forms is excited, some of the energy can subsequently be emitted as fluorescence due to the tendency for matter to relax to its ground state following excitation. The lifetime for excited states depends critically on the type of matter and on the particular state which was excited. With most forms of radiation, such as electrons, $X$ rays, or gamma radiation, a variety of types of excited states are formed during the excitation process and the specturm of fluorescence radiation is complex. With monochromatic lasers it is possible to select just one of the many types of excited states that may be desired. This choice depends on the wavelength tunability of the laser source. In this manner, the fluorescence spectrum may be simplified with laser excitation. 
IONIZATION: Another physical process which may occur when radiation is absorbed in matter is ionization, i.e. the complete removal of an electron from the atom. The process of excitation, usually refers to the promotion of an electron only to discrete levels allowed by quantum mechanics, while for ionization the electron is removed from the atom and can have a continuous range of energy. Sometimes, the ionization process is referred to as a process of excitation into the ionization continuum.

SPECTROSCOPY: Any light source, other forms of radiation, or beams of charged particles such as electrons or positive ions can be characterized with an energy associated with the particles. These energies can be discrete or have a continuous range. In any case the description of the number of particles having particular values of energy is referred to as the spectrum. Spectroscopy in its many forms is derived from this basic concept of the spectrum. Spectroscopy is extremely useful because the spectrum can serve as a most valuable fingerprint. For instance, the spectrum of the light emitted from a gas discharge 1 amp such as a neon sign gives a positive identification of the types of atoms in the lamp. Lasers are used in many forms of spectroscopy based on absorption, emission, scattering, and ionization phenomena. Some of these are described more fulty below, and all of the principal forms of laser spectroscopy are mentioned frequently in this report.

ABSORPTION SPECTROSCOPY: If a continuous spectrum of light illuminates a sample, the transmitted light may be modified; in general, some wavelengths will be more strongly absorbed than others. This absorption spectrum is very useful in identifying the types of atoms or molecules making up the sample.

PHOTOTHERMAL AND PHOTOACOUSTICAL SPECTROSCOPY: Intense laser beams have made possible a form of spectroscopy based on the selective heating of a sample due to the absorption of light at different wavelengths. Actually, this is a subset of absorption spectroscopy and is even more directly indicative of the energy absorbed than is the case just described, where the transmitted light is measured. Photoacoustical or PA spectroscopy is based on the measurement of pressure changes with a sensitive microphone.

RAMAN SPECTROSCOPY: When light scatters from a molecular material, the scattered light can carry away the fingerprints of the molecules present. This is a weak process but provides a valuable method of spectroscopy when using the light intensities available in lasers. The spectrum of scattered light consists of the fundamental or incident wavelength modified to contain other wavelengths that depend on the structure of the molecules. Raman spectroscopy is frequently incorporated into laser systems for remote sensing.

SURFACE-ENHANCED RAMAN SPECTROSCOPY (SERS): When a molecular sample is prepared to be in contact with certain materials, the intensity of the Raman process is greatly enhanced. This effect is believed to be due to the induction of surface excitations called plasmons when the laser beam interacts with the surface-sample interface. The SERS process is being studied in a number of laboratories in an effort to develop improved analytical methods for the analysis of samples such as complex organic molecules.

FLUORESCENCE SPECTROSCOPY: In short times following the absorption of energy by a sample, some of this energy may be emitted as radiation characteristic of the material. Fluorescence spectroscopy based on this process is widely used for detection of atoms and molecules. It is a preferred method for remote sensing and can work even when the target material is being excited by some other agency such as a gas discharge, and in this case provide information on the transient species.

\section{SYNCHRONOUS FLUORESCENCE} SPECTROMETRY: In conventional fluorescence spectrometry, two basic types of spectra can be recorded, i.e., 
an excitation spectrum (fixed excitation wavelength, variable fluorescence wavelength). With the synchronous fluorescence technique, the fluorescence signal is recorded while simultaneously, i.e., synchronously, scanning both excitation and emission wavelengths, maintaining a constant wavelength interval. This technique is characterized by an improved selectivity in the analysis of multicomponent mixtures.

FLUORESCENCE LINE NARROWING SPECTROSCOPY: This technique, which is also described in the literature as "site-selection spectrometry, " is largely used for the characterization of polyaromatic hydrocarbons in view of its special selectivity, since it is performed at very low temperature (about $4 \mathrm{~K}$ ) in organic glasses, where molecules occupy different microenvironments or sites. Excitation by a spectrally narrow laser gives sharp-lined fluorescence spectra, greatly improving resolution.

RESONANCE IONIZATION SPECTROSCOPY (RIS): One of the consequences of energy absorption in a sample is ionization of atoms or molecules. Lasers, especially careful.ly tuned lasers, can be used to selectively ionize the species. In the RIS process, photons are first absorbed to excite the atom, generally according to one-photon selection rules of quantum mechanics. Then, photons are absorbed by the excited atom to produce a free electron and a positive ion. Since the first step is quite selective the entire ionization process is selective. In practice, laser systems are now used to selectively ionize almost any atom in the periodic table.

MULTIPHOTON IONIZATION (MPI) is closely related to RIS but is more general since lasers are now so intense that several photons can be absorbed simultaneously. MPI, therefore, may not be as selective to the type of atom present as is the RIS case. REMPI refers to resonance-enhanced MPI and, thus, is indistinguishable from RIS.
OPTOGALVANIC SPECTROSCOPY: When atoms are already excited, for instance in a flame, lasers can be directed through the sample to enhance the amount of ionization. This process can also be selective as in RIS. Optogalvanic spectroscopy, is commonly used with flames and can be another very sensitive method of ionization spectroscopy.

MASS SPECTROSCOPY (MS): When electrons are used to ionize an atom or a molecule, the positive ions produced are, of course, quite representative of the sample present before ionization. This has led to the discipline of mass spectroscopy, where the mass of the ion is determined by a mass spectrometer. MS is one of the most powerful and sensitive analytical methods in existence. When electrons ionize complex molecules, several mass peaks are formed for each molecule. In MS, mass analys is is done by deflection in a magnetic field, by time-of-flight, or by a variety of other methods. However, regardless of the method, the presence of various mass peaks causes interferences, related to and exacerbated by the fact that no method has perfect mass resolution. If two atoms have the same mass but different atomic numbers, a MS system may not be able to differentiate the two. This is usually referred to as the isobar problem. Since atoms can have a number of stable isotopes, it is possible for two types of atoms to have nearly the same mass and identical mass numbers, i.e., they are isobars. High resolution MS can resolve many of the isobars, provided they are of relatively similar abundance.

RESONANCE IONIZATION MASS SPECTROMETRY (RIMS): Lasers can be used as an ionization source in a mass spectrometer system. When the laser is used in the selective RIS process, the strongest features of both the laser and the mass spectrometer are combined. For instance, the isobar problem is eliminated when detecting atoms. Further, the resulting mass spectra (of organic compounds) are much simpler with 
the resonance ionization process. RIMS systems have been developed which combine laser ionization with virtually all types (magnetic sector, time-of-flight, and quadrupole) of mass spectrometers.

ATOMIZATION: In many laser spectroscopy approaches to trace element analysis, it is necessary first to atomize the sample. For instance, to use RIS, free atoms, representative of the solid, must be produced. Thermal sources in which the sample is heated in a small graphite furnace is often used. In other cases, atoms can be ablated from the solid by focusing a strong laser beam onto the sample. Another method which is better understood and under better control than laser ablation is sputtering of atoms using a beam of charged particles. In principle, the sputtering process is simple: a beam of charged particles imparts considerable kinetic energy to the atoms of a solid. These hot atoms impart energy to several other atoms in a collisional cascade, resulting in the emission of several neutral atoms for each energetic charged particle striking the sample.

SPUTTER - INITIATED RESONANCE IONIZATION SPECTROSCOPY (SIRIS): Sputter initiated resonance ionization spectroscopy is the combination of a sputter source for atomization, RIS for selective ionization, and a mass spectrometer for mass selection. Systems of this type are being used for the understanding of surfaces and solids and are being developed for routine trace element analyses of solids.

OPTRODES: Chemical substances can be deposited on the ends of optical fibers so that when specific other chemicals, such as pollutants interact, light changes in passing through the optical fiber are produced. These detectors, or optrodes, are being developed for a wide variety of applications, and they are becoming an interesting class of specific chemical detector.

CHEMICAL SPECIATION: The term means identification of inorganic, organo- metallic, and organic compounds present in the environment, and emphasizes the fact that one needs to make a clear distinction between the capability of a technique of measuring the total concentration of an element and that of measuring the concentration of each of its chemical forms.

BIOSENSOR: This term, which can be understood in analogy with the term "chemical sensor," can be defined as an analytical device which incorporates a biologically active material in intimate contact with an appropriate transducer for the purpose of detecting, reversibly and selectively, the concentration or activity of chemical species in any type of sample. It can also be defined as an analytical device that responds selectively and reversibly to the concentration or activity of chemical species in biological samples. This last definition refers to the type of sample, while the former refers to the nature of the chemical reaction that generates the analytical signal.

DISSOLVED ORGANIC MATTER (DOM): This term refers to the large and complex organic acids found in natural waters. It is variously called dissolved organic carbon (DOC); humic substances; and Gelbstoffe or, more specifically, fulvic or humic acids. Collectively, these dissolved organic materials represent an important component of the global carbon cycle.

REMOTE SENSING: This term is used for all types of analytical measurements where the sample stays in one location and the measuring instruments stay in another location. Therefore, it refers to "real time" acquisition of the analytical data. In environmental applications, remote sensing with laser sources is called with the acronym LIDAR (1ight detecting and ranging). With the advent of fiber optics and chemical sensors, remote measurements of many physico-chemical parameters have become feasible. 
SENSITIVITY AND SELECTIVITY UNITS: Traditionally, a variety of units have been used for expressing both the sensitivity and the selectivity of analytical methods. With laser spectroscopic methods, it is even possible to discuss the actual counting of atoms. Even with this ultimate in sensitivity, it is still. necessary to put limits on the selectivity, which can be either elemental or isotopic. For instance, one could speak of the detection of one atom of cesium in the background of $10^{19}$ atoms of argon, or the counting of 100 atoms of ${ }^{81} \mathrm{Kr}$ in a mixture of $10^{7}$ atoms of ${ }^{82} \mathrm{Kr}$. In many cases the natural unit is based on the mass of an analyte in a matrix with a much larger mass. For high sensitivity and high selectivity methods, notations such as milligrams, mg $\left(10^{-3} \mathrm{~g}\right)$; micrograms, $\mu \mathrm{g} \quad\left(10^{\circ} \mathrm{g}\right)$; nanograms, ng $\left(10^{-6} \mathrm{~g}\right)$; picogramis, pg $\left(10^{-12} \mathrm{~g}\right)$; femtograms, fg $\left(10^{-15} \mathrm{~g}\right)$; or attograms, ag $\left(10^{10} \mathrm{~g}\right)$ are common. For example, the expression $1 \mathrm{pg} / \mathrm{g}$ means $10^{-12} \mathrm{~g}$ of an analyte in $1 \mathrm{~g}$ of a matrix. The concentration detection limit of $10^{-12} \mathrm{M}$, for example, means that $10^{-12} \mathrm{gram}$ molecular weights of a substance can be detected in 1 liter of material.

ENERGY UNITS: Energy levels of atoms and molecules are frequently expressed in electron volts (eV) or, al ternatively, in wave numbers $\mathrm{cm}^{-1}$. A useful approximate conversion is $1 \mathrm{eV}=$ $8000 \mathrm{~cm}^{-1}$. The energy of light particles or photons is often expressed in terms of wavelength. In this case, a useful conversion is $1 \mathrm{eV}=12345 / \lambda$, where $\lambda$ is the wavelength in Angstrom units and $1 \AA=10^{-8} \mathrm{~cm}$ or $0.1 \mathrm{~nm}$. 


\section{APPENDIX III}

\section{PEER GROUP}

The members of the expert panel are grateful for useful comments and suggestions from the following scientists. We are pleased that these many experts in laser science took time to review and comment on a draft of this report.

Prof. Isaac Abella

Department of Physics

University of Chicago

5734 S. Ellis Avenue

Chicago, Illinois 60637

Prof. Norman Dovichi

Department of Chemistry

University of Alberta

Edmonton, Alberta

CANADA TGG $2 G 2$

Dr. Jack Fassett

A21 Physics

National Inst. of Standards \&

Technology

Gaithersburg, Maryland 20899

Prof. Robin M. Hochstrasser

Department of Chemistry

University of Pennsylvania

Philadelphia, Pennsylvania 19104

Prof. Bruce S. Hudson

Department of Chemistry

University of Oregon

Eugene, Oregon 97403

Dr. Ralph R. Jacobs

Director, Corporate Technical

Development

Spectra-Physics, Inc.

3333 North First Street

San Jose, Cal ifornia 95134-1995

Dr. John C. Miller

MS 6125, B1dg. 4500S

Oak Ridge National Laboratory

P. 0. Box 2008

Oak Ridge, Tennessee 37831-6125

Prof. Dr. K. Niemax

Institut fur Spektrochemie

Postfach 778

4600 Dortmund 1

Federal Republic of Germany
Dr. Stephen L. Palfrey

David Sarnoff Research Center

CN 5300

Princeton, New Jersey 08543-5300

Dr. Marvin G. Payne

MS 6378, Bldg. 5500

Oak Ridge National Laboratory

P. 0. Box 2008

Oak Ridge, TN 37931-6378

Prof. Edward Piepmeir

1-B Barnes, Escandido Village

Stanford, CA 94305

Dr. Richard L. Sheffield

MS-H825

Los Al amos National Laboratory

P. 0. Box 1663

Los Alamos, New Mexico 87545

Dr. Gerald J. Small

Ames Laboratory

Iowa State University

Ames, Iowa 50011

Dr. Andrew C. Tam

Dept. K07-803 E

IBM Almaden Research Center

650 Harry Road

San Jose, Cal ifornia 95120

Prof. J. D. Winefordner

Chemistry Division

University of Florida

Gainesville, Florida 32611

Dr. Edward A. Yeung

Department of Chemistry

Iowa State University

Ames, Iowa 50011 